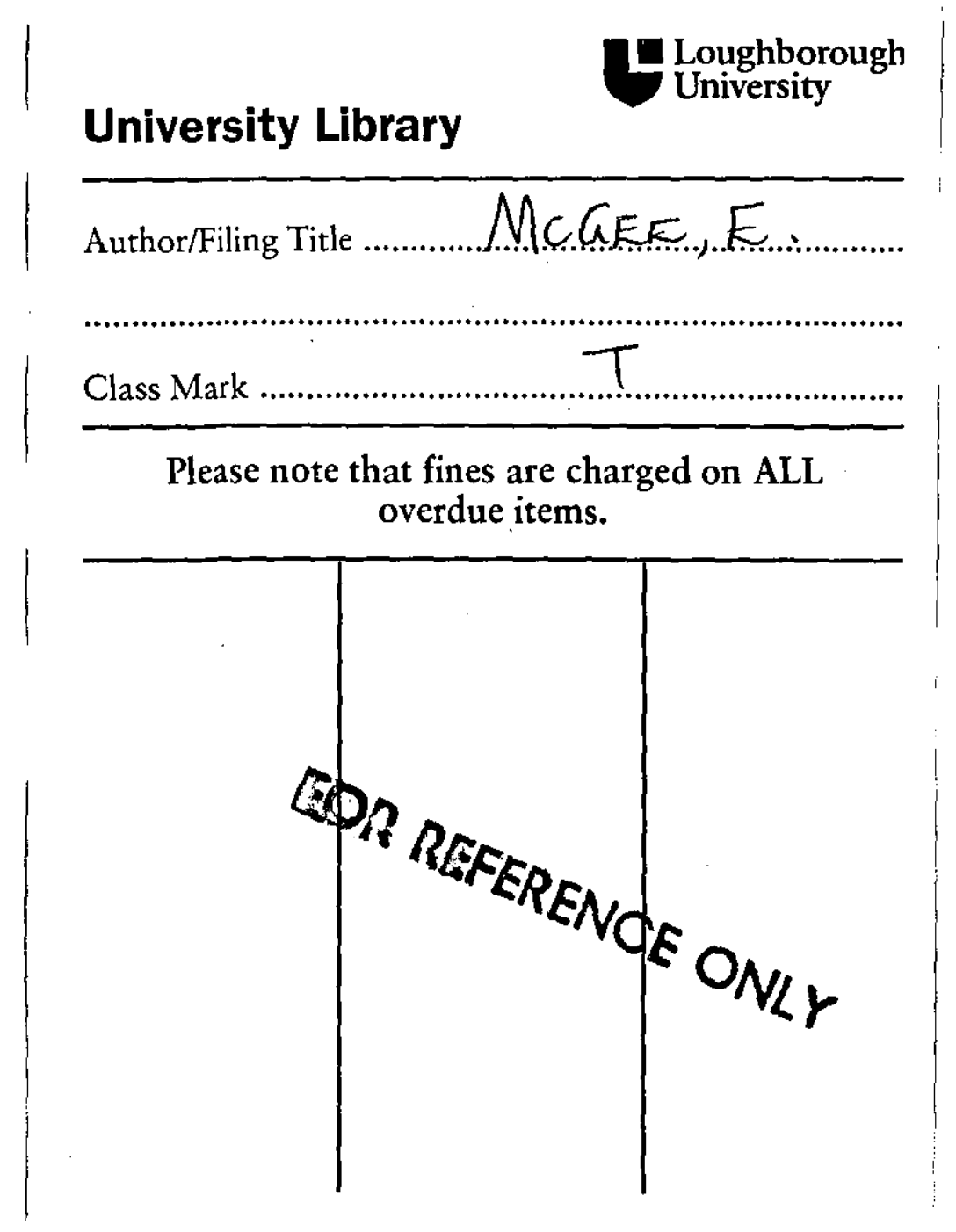

0403411084 



\title{
Multiscale Modelling of Nanoindentation
}

\author{
by \\ Edward McGee
}

\begin{abstract}
A Doctoral Thesis
Submitted in partial fulfilment of the requirements for the award of Doctor of Philosophy of Loughborough University
\end{abstract}

September 2006

(C) Edward McGee 2006 


\begin{tabular}{|c|}
\hline $\begin{array}{l}\text { Loughborough } \\
\text { University } \\
\text { Pilkington Library }\end{array}$ \\
\hline Date Jan 2007 \\
\hline Class \\
\hline
\end{tabular}




\section{Abstract}

The process of nanoindentation causes physical phenomena not only at the nano-scale, but at multiple length scales up to the macroscopic. This thesis investigates multiscale modelling of nanoindentation that links atomistic scale molecular dynamics (MD) to a finite element (FE) model in order to extend the length scales that can be modelled. Existing multiscale models are investigated and the relevant advantages and disadvantages of each are discussed. New coupling techniques are developed in both 2D and 3D, which are applied to nanoindentation test simulations to verify the models.

A new force attribution 3D multiscale model is applied to some studies of nanoindentation of $\mathrm{Au}$ and $\mathrm{Fe}$. The results are compared to those obtained through experiment and to atomistic only models to investigate the effect of the embedding continuum region. These studies show that by extending the length scales, long range effects of nanoindentation can be modelled in the far field by continuum mechanics giving results that are in closer agreement with the experiment. The new coupling method has wide application and a study of laser ablation of $\mathrm{Au}$ has been carried out to show that the multiscale modelling technique can be used to improve the description of this phenomenon also. 


\section{Acknowledgements}

Acknowlegements are made here to those who have supported me during my research in Loughborough. Firstly, I would like to thank both my research supervisors, Roger Smith and Steven Kenny for their helpful discussions and expertise in the development of the work in this thesis. For the experimental results which are discussed in this work I would like to thank our collaborators from UAS, Wildau, and in particular Asta Richter. I would also like to thank Keith Watling for his technical support and assistance.

To my family, and particularly my parents, sister and brothers, thank you for your support during my time at university. And finally, I give a special thank you to Anna, whose positivity has helped me through the last year. 


\section{Contents}

List of Figures vi

List of Tables $\quad$ xvi

1 Introduction $\quad 1$

2 Molecular Dynamics and Finite Elements Methodology 5

2.1 Molecular Dynamics . . . . . . . . . . . . . . 5

2.1.1 Neighbour Lists . . . . . . . . . . . 7

2.1.2 Boundary Conditions ............. 9

2.1.3 Parallel Processing . . . . . . . . . . . . . . . 11

2.2 Interatomic Potentials . . . . . . . . . . . . 12

2.2.1 Lennard-Jones 6-12 Potential . . . . . . . . 13

2.2.2 Ackland Potential . . . . . . . . . . . . 14

2.2 .3 Tersoff Potential .............. 15

2.2.4 Brenner Potential . . . . . . . . . . . 16

2.2.5 Ziegler-Biersack-Littmark Potential ........ 18

2.3 The Finite Element Method . . . . . . . . . . 20

2.3.1 Two Dimensional Finite Elements for Stress Analysis . 20

2.3.2 Three Dimensional Finite Elements for Stress Analysis 25

2.3.3 Boeing-Harwell Packed Matrix Format . . . . . . . . 30 
3 Nanoindentation and Modelling Nanoindentation 33

3.1 Experimental Indentation $\ldots \ldots . \ldots 33$

3.1.1 AFM Cantilever Indentation . . . . . . . . 35

3.2 Atomistic Modelling of Nanoindentation . . . . . . . . 37

3.2.1 The Spring Model for Indentation . . . . . . . . . . 38

3.2.2 Cube Corner Indenter Specification . . . . . . . 40

3.2.3 Finite Temperature Control ............. 41

3.3 Simulation Results ................. 44

3.3.1 Diamond Indentation Results ... . . . . . . . . 44

3.3.2 Silicon Indentation Results . . . . . . . . . . . . 48

3.4 Conclusions ........................ 52

4 Existing Multiscale Models $\quad \mathbf{5 4}$

4.1 The Mixed Finite Element-Atomistic Method . . . . . . . 56

4.2 The Concurrent Coupling of Length Scales Method . . . . . 59

4.3 The Quasicontinuum Method ............. 62

4.4 Conclusions ................... 66

5 Two Dimensional Multiscale Modelling $\quad 68$

5.1 Meshing Technique for Embedding Atomistics in 2D . . . . 69

5.2 Matching Elastic Constants in 2D . . . . . . . . . . 73

5.3 Finite Element Time Integration . . . . . . . . . . . 75

5.4 Initial Coupling Method for up to Second Nearest Neighbours 77 5.4 .1 Methodology .............. 77

5.4 .2 Testing and Results . . . . . . . . . . 80

5.4 .3 Conclusions ................. 83

5.5 Position Matching Generic Coupling Technique in 2D . . . . 85

5.5.1 Methodology .............. 86 
5.5 .2 Testing and Results . . . . . . . . . . . . . 90

5.5 .3 Conclusions . . . . . . . . . . . . . . . 100

6 Three Dimensional Multiscale Modelling 102

6.1 Meshing Technique for Embedding Atomistics in 3D . . . 103

6.2 Matching Elastic Constants in 3D . . . . . . . . . . 105

6.3 Assigning Imaginary Atoms to Elements . . . . . . . . . 106

6.4 Position Matching Generic Coupling Technique in 3D . . . 107

6.4.1 Methodology . . . . . . . . . . . . . . . . 107

6.4.2 Testing and Results . . . . . . . . . . . . . . . 111

6.4 .3 Conclusions . . . . . . . . . . . . . . . 115

6.5 Force Attribution Coupled Model . . . . . . . . . . . . 116

6.5.1 Methodology . . . . . . . . . . . . . . 117

6.5 .2 Testing and Results . . . . . . . . . . . 120

6.5 .3 Conclusions . . . . . . . . . . . . . . . 125

7 Applications of Multiscale Modelling $\quad 126$

7.1 Nanoindentation . . . . . . . . . . . . . . . 126

7.1.1 Au $15 \AA$ Indentation . . . . . . . . . . . . . . 126

$7.1 .2 \quad \mathrm{Fe} 15 \AA$ Indentation . . . . . . . . . . . . . . 133

7.1 .3 Conclusions . . . . . . . . . . . . . . . . 135

7.2 Laser Ablation . . . . . . . . . . . . . . . . . . . 137

7.2.1 Au Laser Ablation . . . . . . . . . . . . . . . 137

7.2 .2 Conclusions . . . . . . . . . . . . . . . . . 140

8 Conclusions and Future Work 145

8.1 Future work . . . . . . . . . . . . . . . 148 


\section{List of Figures}

2.1 A two dimensional representation of a neighbour list. . . . . 8

2.2 An illustration of the cell index method. The entire system is divided into small sub-boxes. To construct the neighbour list for an atom, only atoms in that same sub-box and surrounding sub-boxes need to be considered. . . . . . . . . . . . . 9

2.3 A schematic of a square 2D space in which a molecular dynamics simulation is taking place. Atoms in the lighter central area are free to move under conventional molecular dynamics. Atoms contained in the darker layer at the boundary have fixed positions in space. . . . . . . . . . . . . 10

2.4 Periodic boundary conditions have been applied in the horizontal direction. Although atoms $i$ and $j$ are on opposite sides of the box of atoms, they are considered to be next to each other. . . . . . . . . . . . . . . 11

2.5 Triangular finite element discretisation of an arbitrary shape. . 21

2.6 A typical element with local and global node number shown in circles and squares respectively. . . . . . . . . . . . . 22

2.7 Each element is mapped to this standard element for simplicity of the shape functions in terms of the natural coordinates $\xi$

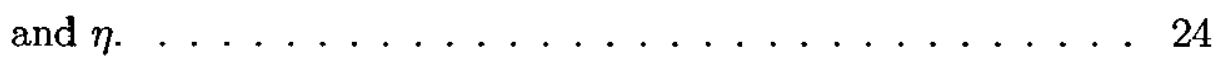


2.8 A typical tetrahedral element with local node numbers. Each node has three degrees of freedom as they can move in the $x$,

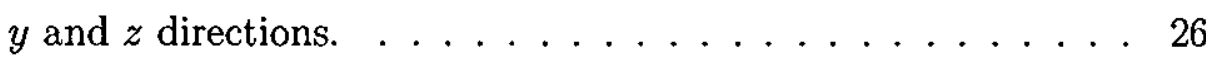

2.9 Each element is mapped to this standard element for simplicity of the shape functions in terms of the natural coordinates $\xi$, $\eta$ and $\zeta \ldots \ldots \ldots \ldots \ldots \ldots \ldots \ldots \ldots \ldots$

3.1 A typical force-depth curve from a nanoindentation experiment. The force on the indenter increases with depth to a maximum force of $P_{\max }$. The indenter is then held fixed for a holding period before being retracted. . . . . . . . . . . . 34

3.2 A schematic of the AFM cantilever system. The laser and photodetector are used to measure the deflection of the end of the cantilever. . . . . . . . . . . . . . . . . . 36

3.3 A pyramidal cantilever used in an AFM, picture courtesy of Professor A. Richter, University of Applied Sciences, Wildau. (a) shows a bottom-up view of the cantilever with the tip at the vertex. (b) shows the cantilever attached to the movable support in the AFM. (c) shows a further magnification of the cube corner tip. . . . . . . . . . . . . . . . . . . . 37

3.4 A schematic representation of the spring model for indentation. The indenter is attached to three supports via springs in the $x, y$ and $z$ directions. The displacement of the supports cause the motion of the indenter. . . . . . . . . . 38

3.5 Typical vertical motion of the indenter during the simulation. The displacement does not always need to return to zero due to plastic deformation. The simulation may be terminated when there is no resultant force on the indenter. . . . . . . . . 39 
3.6 A schematic of the crystal structure of the indenter. A corner is cut along the (111) plane from a cubic diamond lattice with $\{100\}$ surfaces, resulting in an indenter with a $90^{\circ}$ opening angle. 40

3.7 A plan view of an indenter with a truncated tip and dimer reconstructed $\{100\}$ surfaces. . . . . . . . . . . . 41

3.8 A schematic diagram of atom types for a thermalised system. Black atoms are fixed in position during the simulation. The grey atoms are connected to a heat bath which controls the system temperature during the simulation. The white atoms are free unconstrained atoms under the MD methodology. . . . 44

3.9 MD simulation of successive images of the cube-corner diamond indenter as it penetrates the diamond (100) surface showing the indenter compression. The horizontal line is the position of the surface. The images are given after every 100 ps of the indentation process. . . . . . . . . . . . 46

3.10 Force depth curve for the indentation of the cube corner indenter into H-terminated diamond (100). The curve profile shows only elastic deformation until the indenter is removed from the substrate. . . . . . . . . . . . . 47

3.11 The experimental force-depth curve for indentation into diamond $(100) \ldots \ldots \ldots \ldots$. . . . . . . . . . . . . . . .

3.12 Images from various stages of the diamond (100) indentation. The maximum depth is reached after $0.7 \mathrm{~ns} . \ldots 4 . \ldots . .49$

3.13 A top-down view of the indentation into $\mathrm{Si}(100)$ : (a) at the maximum indentation depth of $25 \AA$. (b) after the indenter has been removed. This illustrates the elastic recovery of the substrate when the indenter is extracted. . . . . . . . . . 51 
3.14 Force depth curve for the indentation of the cube corner indenter into H-terminated silicon (100). The curve profile shows extensive plastic deformation during indentation. A strong adhesion between indenter and sample is indicated by the negative force during unloading. . . . . . . . . . . . . 5 52

4.1 A 2D example of a typical coupling setup. The transition region in the middle uses some approximation to overcome the incompatibility of the two methods. . . . . . . . . . . . 56

4.2 A 2D schematic of the FEAt transition region. The white triangular elements in the continuum region use a nonlinear elastic law. The grey elements in the transition region are treated with a non-local modification. . . . . . . . . . . 57

4.3 A 2D schematic of the CLS transition region. Dashed atomistic bonds contribute half of their energy to the systems total energy, as do the grey elements in contact with the continuum boundary. . . . . . . . . . . . . . . . . . . 60

4.4 A 2D schematic of the QC transition region. Particles A and $\mathrm{P}$ experience 'ghost forces' which the QC method balances with a correction term. The grey elements at the interface contribute only part of their energy to the total system energy. 63

5.1 Mesh Generation and Node Density. (a) The node placement and connectivity is completed for an eighth of the continuum region, (b) it is then repeated to embed the square atomistic

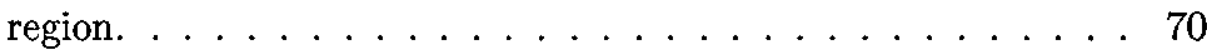


5.2 Node positioning in the trapezium. The number of nodes is reduced at each layer from the inside edge to the outside edge and are spaced equally along each layer. . . . . . . . . 71

5.3 2D tessellation between layers of nodes. (a) The number of nodes in neighbouring layers is equal. (b) The number of nodes is reduced between layers. . . . . . . . . . . . . . 72

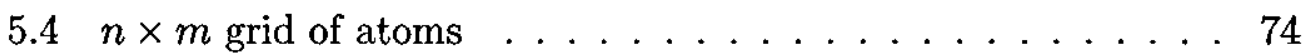

5.5 Elongation of an $n \times m$ grid of atoms due to an applied force at the ends. . . . . . . . . . . . . . . . . . 74

5.6 Calculating the mass of a node. For 2D triangular elements, a third of the mass of the elements is attributed to each of the three nodes. . . . . . . . . . . . . . . . . . . 76

5.7 Schematic of the coupled interface in 2D. FE nodes (X) coincide with MD atoms at the interface. . . . . . . . . 78

5.8 Thin film indentation used to test the 2 D model. . . . . . 81

5.9 Images from the test simulation at various time steps. (a) The strain field can clearly be seen in the MD region, but is yet to cross into the continuum region. (b) The strain field has begun to cross the interface into the continuum region. (c) Larger displacements are visible further into the continuum region. (d) The motion of material due to the indentation generates elastic waves in the continuum region. These are fed back into the atomistic region via the coupled interface. . . . . . . . 84

5.10 Snapshot at 225ps. Interference patterns are visible where elastic waves have crossed one another. . . . . . . . . . 85 
5.11 Snapshot at 400ps. The waves bounce off the outside boundary of the continuum region creating further interference patterns. . . . . . . . . . . . . . . . . . . . 86

5.12 Schematic of the 2D generic coupled interface. The green imaginary atoms are contained within the first layer of elements and provide neighbours of the atoms modelled by MD. . 87

5.13 Images from the strain test simulation taken at 8ps intervals. Both the atomistic and continuum regions are shown on the left and right of each figure respectively and are coloured according to displacement from zero (blue) to $0.2 \AA$ (red). The left hand image is the expanded version from the square in the centre of the right hand image. . . . . . . . . . . . . . . 92

5.14 Images of the continuum region from the strain test simulation taken at 20 ps intervals. . . . . . . . . . . . . . . . . 94

5.15 Images from the strain test simulation with fixed boundary conditions taken at 8 ps intervals. The fixed boundaries prevent the strain field from spreading into the far field. . . . . . 95

5.16 The energy in the atomistic region (blue), the continuum region (green) and the total system energy (red) for the coupled strain field propagation simulation. . . . . . . . . . . 96

5.17 A comparison of the total system energies for the coupled model (red) and the atomistics only model (blue). . . . . . . 96

5.18 The displacement of a face atom during the 2D strain propagation test simulation, calculated by atomistics (blue) and continuum mechanics (red). . . . . . . . . . . . 9 97 
5.19 Images of the atomistic region from the elastic wave test simulation taken at $10 \mathrm{ps}$ intervals. There is very little disturbance after the waves have entered the continuum region. . . . . . 98

5.20 Images of the continuum region from the elastic wave test simulation taken at $10 \mathrm{ps}$ intervals. The elastic wave generated in the atomistic region moves smoothly into the continuum

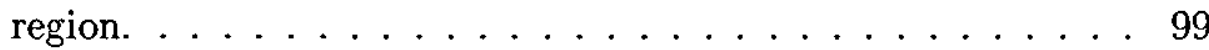

5.21 Images of the atomistic region from the elastic wave test simulation with fixed boundary conditions. The elastic waves are reflected back into the atomistic region and result in interference waves. . . . . . . . . . . . . . . . 100

6.1 A schematic diagram of the initial pillars used to tessellate the continuum region. . . . . . . . . . . . . . . . . . 104

6.2 The cuboidal pillars are stretched to fill the space between them. (a) shows the final shape of pillar a which is also used for pillars b-d. (b) shows the final shape of pillar e. . . . . . . 104

6.3 Embedding an atomistic region within a continuum region for simulating nanoindentation. Typical length scales are shown for the two regions. . . . . . . . . . . . . . . 108

6.4 Atom types of the coupled model. In the atomistic region atoms are coloured yellow, imaginary atoms are coloured green, node-atoms are dark blue and face atoms are light blue. (a) shows all atoms of the coupled model. (b) shows only atoms within the transition region. . . . . . . . . . . . . . 109

6.5 Images from the 3D position matching model test simulation. The strain field spreads throughout the material in both the atomistic and continuum regions. . . . . . . . . . . 113 
6.6 Energy in the atomistic and continuum regions and the total energy of the $3 \mathrm{D}$ position matching method during the simulation. ........................ . . . . . . . . . .

6.7 The comparison of the displacement of a face atom in the transition region. Its actual position computed by MD is shown in blue and the interpolated position due to the FE model is shown in red. . . . . . . . . . . . . . . . . . . 114

6.8 The effect of damping the atoms near the interface to remove the energy increase. (a) shows the energy in the damped and undamped cases. (b) shows only the damped case. . . . . . . . 115

6.9 (a) Cross-section of the 3D coupling interface: The dark blue atoms near the edge of the atomistic region are node-atoms and coincide with FE nodes. The lighter layer of green atoms on the outside of the atomistic region are imaginary atoms. These lie within the first layer of elements in the continuum region. (b) Close-up of the arrangement at the interface. . . . 118

6.10 A 1-D representation of MD interactions at the interface: Atoms 1-4 are free atoms, modelled by MD. Node-atom 5 coincides with the first node of the FE region and atoms 6 and 7 are imaginary atoms within the first element in the FE region. First and second nearest neighbour interactions near the interface are shown. . . . . . . . . . . . . . . 119

6.11 Images of how the strain field due to indentation spreads through the coupled interface. Snap shots are taken at $40 \mathrm{ps}$, 60 ps, 80ps and 100ps. . . . . . . . . . . . . 122 
6.12 Energy in the atomistic and continuum regions and the total energy of the coupled system during the simulation. The energy in the continuum region is shown relative to the total energy. . . . . . . . . . . . . . . . . . . . . . 123

6.13 Kinetic energy in the atomistic and continuum regions. The energy is able to pass from the atomistic region into the continuum region to prevent artificial heating of the atoms. . . . . 124

7.1 (a) The normal force on the indenter versus indentation depth for both the coupled method and the atomistic only simulations. Note that the first pop-in occurs at the same normal force of $\approx 125 \mathrm{nN}$. (b) The change in total system energy during the simulations. . . . . . . . . . . . . 128

7.2 Images of damage due to indentation. (a) and (c) are from the coupled model at maximum indentation depth and after extraction respectively. (b) and (d) are from the atomistic only simulation, also at maximum indentation depth and after extraction respectively. Atoms are coloured according to how much they have been displaced from their original position. . . 131

7.3 Images of slip underneath the indenter. (a) and (c) are from the coupled model at maximum indentation depth and after extraction respectively. (b) and (d) are from the atomistic only simulation, also at maximum indentation depth and after extraction respectively. . . . . . . . . . . . . . . 132

7.4 Graph showing the kinetic energy in the atomistic region during the simulation. Results are shown for both the coupled model and the atomistic only simulation. . . . . . . . . 133 
7.5 Force-depth curve for the $15 \AA$ indentation of Fe with the coupled model. . . . . . . . . . . . . . . . . . . . . 134

7.6 Images from the $15 \AA \mathrm{Fe}$ indentation: (a) during the elastic deformation period, (b) at the maximum indentation depth, and (c) after the indenter has been removed. The material is coloured on displacement from zero (dark blue) to $1.8 \AA$ and above $($ red) . . . . . . . . . . . . . . 136

7.7 Graph showing the energy in the regions of the coupled model and the total system energy during the laser ablation simulation. 138

7.8 Graph showing the kinetic energy in the atomistic region for both the coupled and atomistic only models during the laser ablation simulation. . . . . . . . . . . . . 140

7.9 Graph showing the average particle temperature against distance from the laser application at various times during the laser ablation simulation. The heat wave is seen to travel through the continuum region away from the atomistic region. 140

7.10 Images of the atomistic region of the coupled model during the laser ablation simulation. . . . . . . . . . . . . . 141

7.11 Images of the continuum region of the coupled model during the laser ablation simulation. . . . . . . . . . . . . 142

7.12 Images of the atomistic only simulation during the laser ablation simulation. . . . . . . . . . . . . . . . 143 


\section{List of Tables}

2.1 Boeing-Harwell Packed Format $\ldots \ldots \ldots \ldots$

5.1 First nearest neighbour atomistic and continuum interactions for particles in figure 5.7. The symmetry indicates that Newton's third law is obeyed. . . . . . . . . . . . . . . . 79

5.2 Second nearest neighbour atomistic interactions and first nearest neighbour continuum interactions for particles in figure 5.7. The symmetry indicates that Newton's third law is obeyed. . . 80

5.3 Parameters used for the $2 \mathrm{D}$ thin film indentation test simulation. 82

5.4 Parameters used for the 2D strain field and elastic wave propagation simulations. . . . . . . . . . . . . . . . . 91

6.1 The elastic constants $C_{11}, C_{12}$ and the shear modulus $C_{44}$ are used to fit the potential function and to calculate the Young's modulus, E, and Poisson's ratio, $\nu$ for the FE model. This ensures that the elastic properties in both the atomistic and continuum regions of a multiscale model are matched. . . . . 106

6.2 The system size for the 3D position matching method test simulation. 'Total atoms' is the approximate number of atoms represented by the combined atomistic and continuum regions. 112 
6.3 The system size for the force attribution method test simulation. Total atoms is the approximate number of atoms represented by the combined atomistic and continuum regions. 121

7.1 The coupled system size used for the Au indentation simulation.127

7.2 Effective indentation depths and contact pressures for different metals. Results for $\mathrm{Fe}$ and $\mathrm{Ag}$ are given for indenter displacements of 15 and $5 \AA$ to give comparative hole depths for the Au results. . . . . . . . . . . . . . . . . 129

7.3 Parameters to investigate elastic and plastic deformation due to the indentation. Elastic recovery is given as both a distance and as a percentage of the maximum hole depth. . . . . . . 130

7.4 The coupled system size used for the Fe indentation simulation. 134 


\section{Chapter 1}

\section{Introduction}

Nanoindentation is an experimental process used to investigate dynamically the mechanical properties of materials and their behaviour on the nanoscale. Examples of applications of nanoindentation include the characterisation of thin film coatings and composite nano-materials [1], where the features of the material change on the nano-scale. Molecular dynamics simulations of these experiments have been carried out on a variety of materials including $\mathrm{Fe}[2,3], \mathrm{Ag}[2], \mathrm{C}[4]$ and $\mathrm{Si}[5]$. The atomistic approach used in these simulations allows detailed description of the change from elastic to plastic deformation and the way in which defects are created when undergoing indentation. Experiments such as nanoindentation generate phenomena not only on the nanometre scale due to dislocation emission, but also on a much larger length scale due to stress fields. However, the limitations imposed by current computational power make the volume of material simulated by the atomistic approach too small to model phenomena in the far field.

When modelling the deformation of a solid material, it is important to use a method which accurately describes the resulting effects. Traditionally, molecular dynamics has been used to model dislocations, cracks and grain 
boundaries on the atomistic scale, whereas effects on larger length scales have been modelled by the finite element method. However, either of these methods alone would be insufficient to model the effects on the different scales, and hence, in recent years, an effort has been made to bridge the length scales via coupled multiscale models. These hybrid models take two or more existing modelling techniques that describe a system on different scales, for example the nanometre and micron length scales, and combine them to produce a model which applies the different techniques to the appropriate part of the system.

Molecular dynamics is a numerical technique which can be used to model solid materials on the atomistic scale. The technique models each atom individually and by numerically solving Newton's equations of motion (a coupled system of ordinary differential equations) the system is evolved through time. Interatomic potential functions are used to calculate applied forces on atoms. The forces are then used with a numerical integration technique to move a step forward in time. Due to high frequency vibrations of molecules, the accurate description of motion requires a very short time step of the order of femtoseconds. Consequently, due to time restrictions, simulations are currently restricted to system sizes of the order of millions of molecules and the simulation time to the order of nanoseconds.

The finite element method is a widely used numerical technique for solving a variety of engineering problems. A continuous complex region is discretised into simple shapes called elements that represent a small part of the overall system. Appropriate physical laws are then applied to the elements such that a solution may be obtained at the vertices of the elements. The arbitrary size and shape of the elements makes the method suitable for a wide range of applications including the deformation and stress analysis of solid materials. 
This work considers multiscale modelling of nanoindentation, coupling a non-local atomistic description of the material under investigation to a local continuum description of the material in the far field. All of the existing and new methods discussed model the plastic deformation in the atomistic region by molecular dynamics, and in the continuum region the finite element method is implemented to model the long range effects of the experiment.

The different modelling techniques applied to regions coupled together in the multiscale models in this thesis are described in detail in chapter 2. The methodology of the molecular dynamics model is given along with the interatomic potential functions used to calculate atomic forces for the materials studied in this work. The chapter gives the derivation of the matrix method for 2D and 3D constant strain finite element methods which may be used to model linear elasticity in solid materials.

Chapter 3 gives an overview of experimental nanoindentation and goes on to discuss the atomistic modelling approach to simulating the experiment. The spring method used in existing atomistic only models of nanoindentation is described and two examples of these simulations are given for diamond and silicon. The results of these experiments are compared to experimental results to verify the requirement of a multiscale model.

The existing 3D multiscale models in the literature which couple atomistics to continuum mechanics are discussed in chapter 4 . The advantages and disadvantages of each of the methods are outlined and conclusions drawn in order to pave the way for the development of new $2 \mathrm{D}$ and $3 \mathrm{D}$ multiscale coupling techniques in chapters 5 and 6 respectively. Initially the development work is undertaken in 2D as the methodology is generally more simple to implement than in the 3D models, and fewer particles need to be modelled, both of which ease the debugging process. The ideas are then extended into 
a fully 3D model where further developments are made to the technique so that the multiscale model can be applied to simulations of real experiments.

The simulation results of nanoindentation into $\mathrm{Au}$ and $\mathrm{Fe}$ using the new multiscale model are discussed in chapter 7 . The results of these simulations are compared to atomistic only simulations and to the experimental results to investigate the effect of the embedding continuum region. Also, a laser ablation of Au simulation is carried out to investigate the model's application to another type of experiment.

Finally, chapter 8 draws conclusions from the work in this thesis. The new coupling techniques developed in this work and the results from the simulations using the models are analysed and discussed, and future work in the field of multiscale modelling is suggested. 


\section{Chapter 2}

\section{Molecular Dynamics and Finite Elements Methodology}

\subsection{Molecular Dynamics}

Molecular dynamics is a numerical technique used to model solids, liquids and gases at the atomic level. Each atom in the system is considered individually as a point mass and by solving Newton's equations of motion (a coupled system of ordinary differential equations), the forces, positions and trajectories of the particles are repeatedly updated, evolving the system through time. The positions of all of the atoms are used to calculate applied forces on atoms via interatomic potential functions. The forces are then used with numerical integration to move a step forward in time. Due to high frequency vibrations of molecules, the accurate description of motion requires a very short time step of the order of femtoseconds. Consequently, due to time restrictions, simulations are restricted to system sizes of the order of millions of molecules and the simulation time to the order of nanoseconds.

It is important that the numerical integration algorithm employed to solve 
Newton's equations of motion is symplectic, i.e. preserves the Hamiltonian invariants to the same order of accuracy as the method. This also ensures that no energy drift in the system occurs. One such method is the velocity Verlet algorithm [6] derived from Taylor series expansions of an atom's position $\mathbf{r}$, its velocity $\mathbf{v}$ and the atom's acceleration $\mathbf{a}$. The update functions to move from time $t$ to time $t+\delta t$ are

$$
\begin{aligned}
\mathbf{r}(t+\delta t) & =\mathbf{r}(t)+\delta t \mathbf{v}(t)+\frac{1}{2} \delta t^{2} \mathbf{a}(t) \\
\mathbf{v}(t+\delta t) & =\mathbf{v}(t)+\frac{1}{2} \delta t[\mathbf{a}(t)+\mathbf{a}(t+\delta t)]
\end{aligned}
$$

where the acceleration is defined as the ratio of the force on the atom to the mass of the atom, $\mathbf{a}(t)=\frac{\mathbf{F}(t)}{m}$. The velocity update equation 2.2 requires the atoms updated acceleration vector from time $t+\delta t$. This is available before the velocity update as it is only dependent on the atom positions that are obtained from information from the previous step. The order of computation is as follows:

- Time step $t$ is complete and $\mathbf{r}(t), \mathbf{v}(t)$ and $\mathbf{a}(t)$ are known for all atoms.

- All atom positions are updated by equation 2.1 .

- Half of the velocity update is calculated using the known accelerations,

$$
\mathbf{v}\left(t+\frac{1}{2} \delta t\right)=\mathbf{v}(t)+\frac{1}{2} \delta t \mathbf{a}(t)
$$

- The updated forces and hence accelerations are calculated from the updated positions $\mathbf{r}(t+\delta t)$.

- The fully updated velocities are calculated for all molecules,

$$
\mathbf{v}(t+\delta t)=\mathbf{v}\left(t+\frac{1}{2} \delta t\right)+\frac{1}{2} \delta t \mathbf{a}(t+\delta t)
$$

- Time step $t+\delta t$ is complete and $\mathbf{r}(t+\delta t), \mathbf{v}(t+\delta t)$ and $\mathbf{a}(t+\delta t)$ are known for all atoms. 


\subsubsection{Neighbour Lists}

In a molecular dynamics simulation, the most computationally expensive operation is calculating the interatomic forces. Theoretically, at every step of the simulation all atoms should be considered to calculate the force field, which for a system of $N$ atoms makes this an $O\left(N^{2}\right)$ operation. For large systems, this method to calculate the forces is unsuitable as each time step would take too long. In reality, for most uncharged systems, there is a separation between atoms beyond which the resulting force is small enough that it may be neglected. Interatomic potentials which describe the forces for these sorts of materials make use of this by having a cut-off radius. As the separation distance approaches this cut-off radius, both the energy and force also approach zero. For separation distances greater than or equal to the cut-off radius the energy and force are zero, i.e. the atom is only interacting with other atoms within its cut-off radius.

To increase computational efficiency, a neighbour list is constructed for each atom. This is a list of atoms that lie within a distance of the cutoff radius $r_{c}$, plus an outer skin of $r_{s}$, illustrated in figure 2.1. Without this outer skin the neighbour lists would need to be updated at every time step as an atom may have moved within the cut-off radius of another atom. However, also including atoms within the outer skin allows a certain amount of movement of atoms before the neighbour list should be re-evaluated. Now to calculate the force for an atom, only atoms in its neighbour list need to be considered, making the process an $O(N)$ operation. To choose the thickness of the skin it should be noted that for smaller $r_{s}$ the neighbour list needs to be updated more frequently, but for larger $r_{s}$ more interactions are considered in the force calculation. Relative to the cut-off radius $r_{c}$, typical skin thicknesses are $\approx 5 \%$. 


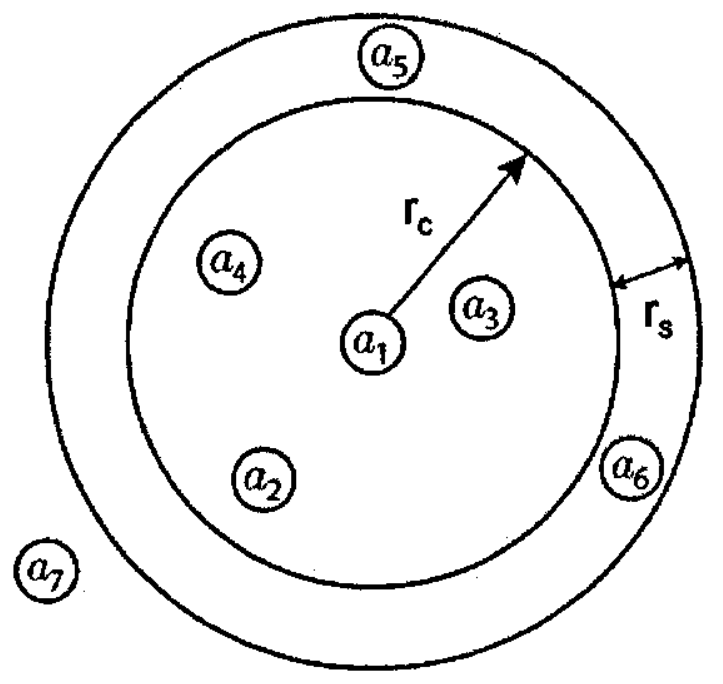

Figure 2.1: A two dimensional representation of a neighbour list [7] for atom $a_{1}$. Atoms $a_{2}, a_{3}$ and $a_{4}$ are within $r_{c}$ of atom $a_{1}$ and therefore are included in the neighbour list and interact with this atom. Atoms $a_{5}$ and $a_{6}$ have a separation distance between $r_{c}$ and $r_{c}+r_{s}$ from atom $a_{1}$ and therefore are included in the neighbour list but do not interact with this atom. Atom $a_{7}$ has a separation distance greater than $r_{c}+r_{s}$ from atom $a_{1}$ and therefore is not included in the neighbour list and does not interact with this atom.

\section{Cell Index Method}

Although having a neighbour list considerably optimises the force calculation at each time step, the actual construction of neighbour lists is still very computationally expensive. Again each pair of atoms must be considered to decide whether they should be in each others list, and hence is an $O\left(N^{2}\right)$ operation. In order to speed up the construction of neighbour lists the cell index method [8] can be used. A lattice of atoms with sides of length $L_{x}, L_{y}$ and $L_{z}$ is broken down into an $M_{x} \times M_{y} \times M_{z}$ array of sub-boxes whose sides must be greater than the potential cut-off distance plus the skin thickness. A 
separate list of atoms is stored for each sub-box, and as illustrated in figure 2.2 , when constructing the neighbour list for a particular atom, only atoms in the same sub-box and surrounding sub-boxes need to be checked, making the process an $O(N)$ operation.

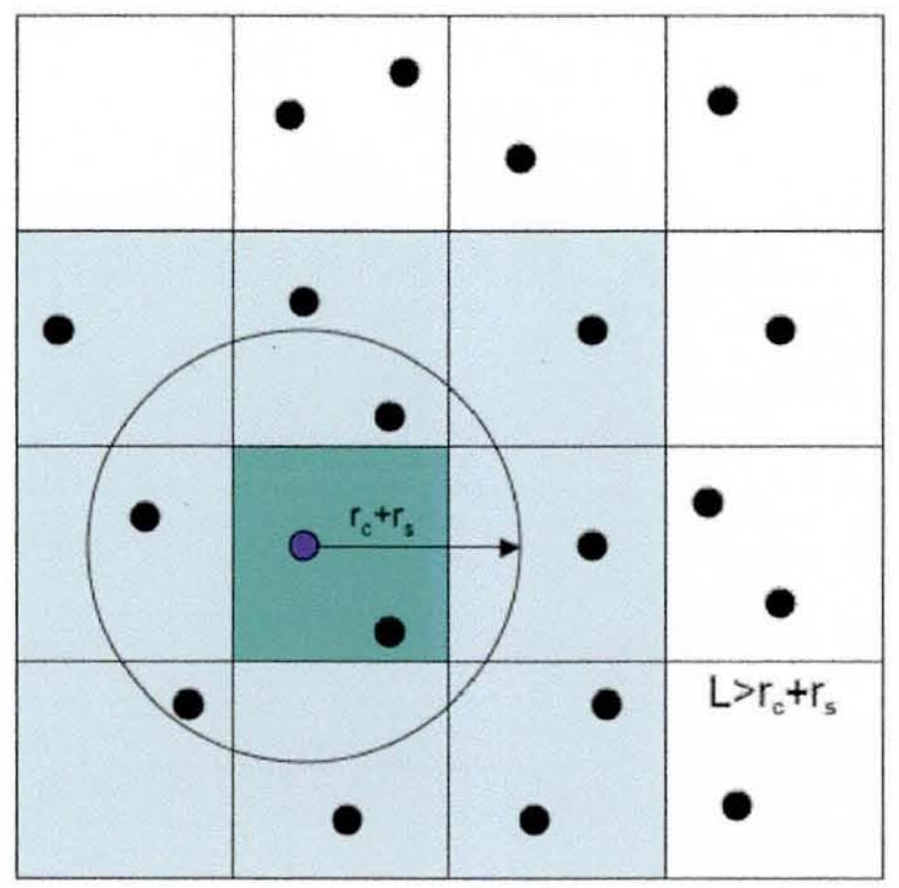

Figure 2.2: An illustration of the cell index method [8]. The entire system is divided into small sub-boxes. To construct the neighbour list for an atom, only atoms in that same sub-box and surrounding sub-boxes need to be considered.

\subsubsection{Boundary Conditions}

Molecular dynamics simulations involve a finite number of atoms and hence take place within a finite space. For simulations of bulk material it is undesirable to leave the atoms at the boundaries or surfaces to interact and move 
freely in the normal way as they may drift apart during the simulation, so boundary conditions may be put in place to prevent this from occurring. A simple technique is to use fixed boundary conditions (FBC) where a layer of atoms at the boundary still interact with their neighbours, but are held fixed in space as illustrated in figure 2.3. The thickness of the fixed layer should be made large enough so that all atoms allowed to move freely have a full complement of neighbours up to the potential cut-off distance.

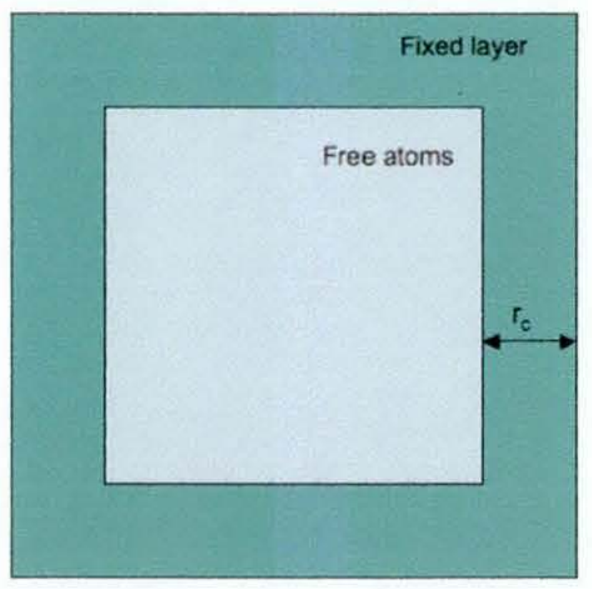

Figure 2.3: A schematic of a square 2D space in which a molecular dynamics simulation is taking place. Atoms in the lighter central area are free to move under conventional molecular dynamics. Atoms contained in the darker layer at the boundary have fixed positions in space.

For simulations where fixing the boundary atoms is undesirable and working with a much larger space would be preferred, periodic boundary conditions (PBC) may be implemented. In doing so, an infinite space is assumed to be repeatedly tiled with identical copies of the box of atoms in the simulation in any or all of the three dimensions.

Using PBC changes the way in which atoms interact with other atoms 
and also the way in which atoms positions are updated. For example, an atom near the boundary in a certain direction that is employing $\mathrm{PBC}$ is considered to be next to atoms at the opposite boundary in that direction, as illustrated in figure 2.4 and must include interactions with those atoms in the force calculation. Also, an atom that moves across the boundary in a direction employing $\mathrm{PBC}$ will reappear at the opposite boundary in that direction.

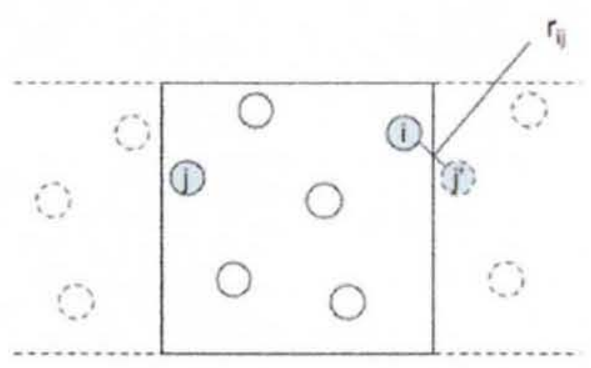

Figure 2.4: Periodic boundary conditions have been applied in the horizontal direction. Although atoms $i$ and $j$ are on opposite sides of the box of atoms, they are considered to be next to each other.

\subsubsection{Parallel Processing}

Although the molecular dynamics method is an extremely accurate way to model a material, it is also a computationally intensive process. This is due to the small time steps necessary to model the high frequency atomic vibrations and the large number of atoms involved. The latter of these reasons may be addressed by distributing the atoms between multiple processors which work on different calculations in parallel.

An efficient method to divide the system across processors is spatial decomposition (SD). The space in which the atoms exist is split into $P$ smaller 
cuboidal spaces where $P$ is the number of processors. Atoms are assigned to processors depending on which of the spaces they lie within, then that processor is responsible for performing the force calculation for each of its atoms. Atoms at the edge of each of the spaces may be neighbours of atoms on another processor and so information about these atoms must be passed between processors to complete the force calculations. However, with spacial decomposition, message passing is kept to local communication as information must only be passed to neighbouring spaces.

During a simulation, atoms may move from one SD region to another. In accordance, processors to which atoms are assigned must also change. This reassignment should be done in conjunction with the updating of the neighbour lists (see section 2.1.1) which will exist for the lists of atoms on each processor.

\section{$2.2 \quad$ Interatomic Potentials}

The numerical integration algorithms used to evolve molecular dynamics systems forward in time are functions of the interatomic forces of the atoms in the system. In classical molecular dynamics, these forces are evaluated via approximate empirical interatomic potential functions. To develop an empirical potential, a functional form is assumed, then the parameters of the function are fitted to either experimental data or results obtained from a more accurate (but also more computationally expensive) method, for example $a b$ initio or tight binding.

The total potential energy of the system, $V$, is a function of the position vectors of each of the $N$ atoms in the system. Hence,

$$
V=V\left(\mathbf{r}_{1}, \mathbf{r}_{2}, \mathbf{r}_{3}, \ldots, \mathbf{r}_{N}\right)
$$


where $\mathbf{r}_{i}$ is the position vector of atom $i$. Now the force vector, $\mathbf{F}_{i}$, acting on atom $i$ can be written in terms of the first partial derivative of the potential function with respect to its position vector as,

$$
\mathbf{F}_{i}=-\frac{\partial V}{\partial \mathbf{r}_{i}}
$$

\subsubsection{Lennard-Jones 6-12 Potential}

The Lennard-Jones 6-12 potential [9] is a simple pairwise function which was initially developed to represent noble gases. Atoms in a noble gas in its solid state are only slightly distorted from the stable closed-shell configuration they have in the free-state, and hence a dipole type interaction can be used to represent the small distortions with an attractive potential proportional to $r^{-6}$ where $r$ is the interatomic spacing. The repulsive part of the potential must be stronger than the attractive part at short distances, and is therefore assumed (for no particular physical reason) proportional to $r^{-12}$. The potential function is given as

$$
V(r)=4 \epsilon\left[\left(\frac{\sigma}{r}\right)^{12}-\left(\frac{\sigma}{r}\right)^{6}\right]
$$

where $\epsilon$ and $\sigma$ are parameters of the potential function which can be adjusted to fit a certain material. They are related to the well depth of the curve and the interatomic equilibrium separation respectively.

Extending the Lennard-Jones 6-12 potential to fit bulk crystal properties, the potential is assumed pairwise additive where the energy of the crystal is given as

$$
U=\sum_{i=1}^{N} \sum_{j=i+1}^{N} V\left(r_{i j}\right)
$$

where $N$ is the number of atoms in the crystal and $r_{i j}$ is the separation distance between atom $i$ and atom $j$. 
In this work, the Lennard-Jones $6-12$ potential has been used as a first approximation during model development to describe the interatomic interactions between particles in molecular dynamics simulations in sections 5.4 , 5.5, 6.4 and 6.5. A cubic function with continuous position and first derivative has been used to take the potential and force values smoothly to zero between first and second nearest neighbours so that near equilibrium in the face centered cubic (fcc) lattice structure, only first nearest neighbours need to be considered in the neighbour list and force calculation.

\subsubsection{Ackland Potential}

The Ackland potential is a many-body potential based on the embedded atom method (EAM) [10]. The empirical data used for the parameterisation are; the lattice parameter, the cohesive energy, the elastic constants, the vacancy formation energy and the stacking fault energy. The method has generally been used for pure metals, for example $\mathrm{Cu}, \mathrm{Ag}, \mathrm{Au}$ and $\mathrm{Ni}$ [11], and $\mathrm{Fe}$ [12]. The potential energy function, $U$, for a system of atoms is made up of two parts. The pairwise function, $V\left(r_{i j}\right)$, describes the repulsive interactions between the nuclei and the embedding function, $F\left(\rho_{i}\right)$, can be thought of as the attractive electron density around each atom. The total potential energy is given by

$$
U=\sum_{i=1}^{N}\left[\sum_{j=i+1}^{N} V\left(r_{i j}\right)+F\left(\rho_{i}\right)\right]
$$

where $r_{i j}$ is the separation distance of two atoms $i$ and $j$ and

$$
\rho_{i}=\sum_{j \neq i} \phi\left(r_{i j}\right)
$$

The functional forms assumed in the Ackland potential take the form

$$
V\left(r_{i j}\right)=\sum_{k} a_{k}\left(r_{k}-r_{i j}\right)^{3} H\left(r_{k}-r_{i j}\right)
$$




$$
\begin{aligned}
& F\left(\rho_{i}\right)=-\sqrt{\rho_{i}}+c_{2} \rho_{i}^{2}+c_{4} \rho_{i}^{4} \\
& \phi\left(r_{i j}\right)=\sum_{k} A_{k}\left(R_{k}-r_{i j}\right)^{3} H\left(R_{k}-r_{i j}\right)
\end{aligned}
$$

where $H$ is the Heaviside unit-step function and $c_{2}$ and $c_{4}$ are fitted constants of the embedding function. The cut-off distance, $r_{c}$ for the Ackland potential can be taken to be the greatest of the $R_{k}$ and $r_{k}$ values as $V\left(r_{i j}\right)=\phi\left(r_{i j}\right)=0$ for all $r_{i j} \geq r_{c}$. The force calculation may then be optimised by employing the neighbour list scheme described in section 2.1.1.

\subsubsection{Tersoff Potential}

The Tersoff potential $[13,14]$ was first used to model covalent elements such as $\mathrm{Si}$ and $\mathrm{C}$, but can also be adapted to other systems. The energy function, $V$, is written as a sum of interactions between pairs of atoms

$$
V=\frac{1}{2} \sum_{i \neq j} \phi_{i j},
$$

where

$$
\phi_{i j}=f\left(r_{i j}\right)\left[V_{R}\left(r_{i j}\right)-b_{i j} V_{A}\left(r_{i j}\right)\right],
$$

$V_{R}$ is a repulsive pair term, $V_{A}$ is an attractive pair term, $r_{i j}$ is the separation distance between atoms $i$ and $j$, and the many-body function $b_{i j}$ is effectively a bond order term. The attractive and repulsive components are given as

$$
\begin{aligned}
& V_{R}\left(r_{i j}\right)=A_{i j} \mathrm{e}^{-\lambda_{i j} r_{i j}} \\
& V_{A}\left(r_{i j}\right)=B_{i j} \mathrm{e}^{-\mu_{i j} r_{i j}} .
\end{aligned}
$$

The repulsive term is pairwise, however, the attractive term is multiplied by the many-body term in order to account for the local environment of atom $i$. The many-body term is given as

$$
b_{i j}=\chi_{i j}\left(1+\beta_{i}^{\eta_{i}} \zeta_{i j}^{\eta_{i}}\right)^{-\frac{1}{2 \eta_{i}}}
$$


where the competition between bonds is described by

$$
\zeta_{i j}=\sum_{k \neq i, j} f\left(r_{i k}\right) \omega_{i k}\left(1+\frac{c_{i}^{2}}{d_{i}^{2}}-\frac{c_{i}^{2}}{d_{i}^{2}+\left(h_{i}-\cos \theta_{i j k}\right)^{2}}\right)
$$

where $\theta_{i j k}$ is the bond angle at atom $i$ between bonds $i j$ and $i k$.

The function $f\left(r_{i j}\right)$, is a cut-off function which takes the potential smoothly to zero between the first and second neighbours and is defined by

$$
f\left(r_{i j}\right)= \begin{cases}1 & r_{i j} \leq R_{i j} \\ \frac{1}{2}+\frac{1}{2} \cos \left[\frac{\pi\left(r_{i j}-R_{i j}\right)}{S_{i j}-R_{i j}}\right] & R_{i j}<r_{i j}<S_{i j} \\ 0 & r_{i j} \geq S_{i j} .\end{cases}
$$

The values for $\lambda_{i j}$ and $\mu_{i j}$ are calculated by the arithmetic means, $\lambda_{i j}=\frac{1}{2}\left(\lambda_{i}+\right.$ $\left.\lambda_{j}\right)$ and $\mu_{i j}=\frac{1}{2}\left(\mu_{i}+\mu_{j}\right)$. The values for $A_{i j}, B_{i j}, R_{i j}$ and $S_{i j}$ are calculated by the geometric means, $A_{i j}=\sqrt{A_{i} A_{j}}, B_{i j}=\sqrt{B_{i} B_{j}}, R_{i j}=\sqrt{R_{i} R_{j}}$, and $S_{i j}=\sqrt{S_{i} S_{j}}$.

The parameters $A_{i}, B_{i}, \lambda_{i}, \mu_{i}, \chi_{i j}, \omega_{i j}, \beta_{i}, \eta_{i}, c_{i}, d_{i}, R_{i}$ and $S_{i}$ are dependant on the atom $i$, or the pair of atoms $i$ and $j$ that are being modelled. A modification to these parameters of the potential [15] allows C-Si-H systems to be modelled by a combined Tersoff-Brenner empirical potential, where $\mathrm{C}-\mathrm{Si}$ and $\mathrm{Si}-\mathrm{Si}$ interactions are modelled by the Tersoff potential.

\subsubsection{Brenner Potential}

The Brenner potential $[16,17]$ has been developed to model carbon systems and fits the graphite and diamond lattice structures quite well. The combined C-Si-H model [15] used in the simulations in chapter 3 uses the Brenner potential to describe the interatomic forces for $\mathrm{C}-\mathrm{C}, \mathrm{C}-\mathrm{H}$ and $\mathrm{H}-\mathrm{H}$ pairs.

The potential function $V$ is taken to be the sum of all pairwise covalent interactions, where the surrounding environment is described by a many- 
body bond-order function, $\bar{B}_{i j}$, and can be written as

$$
V\left(r_{i j}\right)=\sum_{i} \sum_{j>i}\left[V_{R}\left(r_{i j}\right)-\bar{B}_{i j} V_{A}\left(r_{i j}\right)\right]
$$

where the repulsive and attractive parts of the pairwise interactions are given by the functions

$$
V_{R}\left(r_{i j}\right)=f_{i j}\left(r_{i j}\right) \frac{D_{i j}^{e}}{S_{i j}-1} \mathrm{e}^{-\sqrt{2 S_{i j}} \beta_{i j}\left(r_{i j}-R_{i j}^{e}\right)}
$$

and

$$
V_{A}\left(r_{i j}\right)=f_{i j}\left(r_{i j}\right) \frac{D_{i j}^{e} S_{i j}}{S_{i j}-1} \mathrm{e}^{-\sqrt{\frac{2}{s_{i j}}} \beta_{i j}\left(r_{i j}-R_{i j}^{e}\right)} .
$$

The potential function well depth $D_{i j}^{e}$, the equilibrium separation distance $R_{i j}$ and the parameters $S_{i j}$ and $\beta_{i j}$ are dependent on the pair of atoms interacting. The cut-off function $f_{i j}\left(r_{i j}\right)$ smoothly reduces the energy and forces to zero between first and second nearest neighbour distances by the piecewise function

$$
f_{i j}\left(r_{i j}\right)= \begin{cases}1 & r_{i j} \leq R_{i j}^{(1)} \\ \frac{1}{2}+\frac{1}{2} \cos \left[\frac{\pi\left(r_{i j}-R_{i j}^{(1)}\right)}{R_{i j}^{(2)}-R_{i j}^{(1)}}\right] & R_{i j}^{(1)}<r_{i j}<R_{i j}^{(2)} \\ 0 & r_{i j} \geq R_{i j}^{(2)} .\end{cases}
$$

The bond-order function, $\bar{B}_{i j}$ in equation 2.21 is given by the average of the terms for each of the atoms in the bond $i$ and $j$, plus a correction term:

$$
\bar{B}_{i j}=\frac{B_{i j}+B_{j i}}{2}+F_{i j}\left(N_{i}^{(t)}, N_{j}^{(t)}, N_{i j}^{c o n j}\right)
$$

where

$\left.B_{i j}=\left[1+\sum_{k \neq i, j} G_{i}\left(\theta_{i j k}\right) f_{i k}\left(r_{i k}\right) \mathrm{e}^{\alpha_{i j k}\left[\left(r_{i j}-R_{i j}^{(e)}\right)-\left(r_{i k}-R_{i k}^{(e)}\right)\right.}\right]+H_{i j}\left(N_{i}^{(H)}, N_{i}^{(C)}\right)\right]^{-\delta_{i}}$.

The total number of neighbours of atom $i, N_{i}^{(t)}$ is the sum of the number of $\mathrm{C}$ neighbours and $\mathrm{H}$ neighbours, $N_{i}^{(C)}$ and $N_{i}^{(H)}$ respectively, made into smooth 
functions through the use of cut-off functions. $N_{i j}^{c o n j}$ depends on whether a bond between $i$ and $j$ is part of a conjugated system. $G_{i}\left(\theta_{i j k}\right)$ is a function of the bond angle between bonds $i j$ and $i k$, and the functions $H_{i j}$ and $F_{i j}$ are two and three dimensional cubic spline functions respectively, to interpolate between values at discrete numbers of neighbours.

\subsubsection{Ziegler-Biersack-Littmark Potential}

A potential developed by Ziegler, Biersack and Littmark (ZBL) [18] is designed to model interactions at small interatomic separation distances. When a pair of atoms are very close together the strong repulsive force between the two nuclei dominate any other interaction and may be described by a Coulombic potential. As the nuclei come further apart, the surrounding electron field begins to have a screening effect on the repulsive force and hence the ZBL potential defines a screening function, $\phi\left(r_{i j}\right)$ to adjust the Coulombic force. The potential function for two atoms $i$ and $j$ is given by

$$
V\left(r_{i j}\right)=\frac{1}{4 \pi \epsilon_{0}} \frac{Z_{1} Z_{2}}{r_{i j}} \phi\left(r_{i j}\right)
$$

where $Z_{1}$ and $Z_{2}$ are the atomic numbers of the atoms, $r_{i j}$ is the interatomic separation distance of the atoms in $\AA$, and $\epsilon_{0}$ is the electrical permittivity of free space.

For the ZBL potential, the screening function is defined as

$$
\begin{aligned}
\phi(r)= & 0.18175 \mathrm{e}^{-3.1998 r / a_{u}}+0.50986 \mathrm{e}^{-0.94229 r / a_{u}}+0.28022 \mathrm{e}^{-0.4029 r / a_{u}} \\
& +0.028171 \mathrm{e}^{-0.20162 r / a_{u}}
\end{aligned}
$$

where

$$
a_{u}=\frac{0.468377}{Z_{1}^{0.23}+Z_{2}^{0.23}} \AA
$$




\section{Connecting Splines}

The ZBL potential should only be used to describe the repulsive forces at

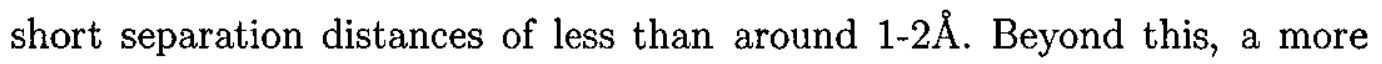
suitable potential should be chosen to model accurately the material under investigation, and hence some method to link the potential functions is required.

The simulations in chapter 3 use connecting spline functions to connect smoothly the ZBL potential to the Tersoff and Brenner potentials at longer ranges. The piecewise potential function is defined as

$$
V(r)= \begin{cases}V_{L}(r) & r<r_{L} \\ V_{S}(r) & r_{L} \leq r<r_{U} \\ V_{U}(r) & r \geq r_{U}\end{cases}
$$

where the ZBL potential is used for $V_{L}(r)$ and the Tersoff or Brenner potential is used for $V_{U}(r)$ depending on what material is being investigated.

The spline function has an exponential form to give a constantly decreasing value over the required range and is given by

$$
V_{S}(r)=\mathrm{e}^{B_{0}+B_{1} r+B_{2} r^{2}+B_{3} r^{3}+B_{4} r^{4}+B_{5} r^{5}}
$$

where the coefficients are determined by solving the simultaneous equations of continuity for position, direction and curvature at both ends of the spline to give continuous position and direction of energy and force, i.e.

$$
\begin{aligned}
V_{S}\left(r_{L}\right) & =V_{L}\left(r_{L}\right) \\
V_{S}^{\prime}\left(r_{L}\right) & =V_{L}^{\prime}\left(r_{L}\right) \\
V_{S}^{\prime \prime}\left(r_{L}\right) & =V_{L}^{\prime \prime}\left(r_{L}\right) \\
V_{S}\left(r_{U}\right) & =V_{U}\left(r_{U}\right) \\
V_{S}^{\prime}\left(r_{U}\right) & =V_{U}^{\prime}\left(r_{U}\right)
\end{aligned}
$$




$$
V_{S}^{\prime \prime}\left(r_{U}\right)=V_{U}^{\prime \prime}\left(r_{U}\right)
$$

\subsection{The Finite Element Method}

The finite element method has become a widely used numerical technique for solving a variety of engineering problems. A continuous complex region is discretised into simple shapes called elements that represent a small part of the overall system. Appropriate physical laws are then applied to the elements such that a solution may be obtained at the vertices of the elements. The arbitrary size and shape of the elements makes the method suitable for applications of deformation and stress analysis of solid materials for example in aircraft or bridge design, or for field analysis of fluid flow, magnetic flux or heat flux. Like any numerical method, the solution is only an approximation of the true solution, however the finite element method allows more accurate solutions to be obtained simply by increasing the number of elements in the same space.

\subsubsection{Two Dimensional Finite Elements for Stress Anal- ysis}

A two-dimensional region, shown in figure 2.5 , is discretised into triangles which almost entirely tessellate the region. The area around the outside which is not filled by the triangles can be reduced by using smaller triangles and is part of the approximate nature of the method. The points where the vertices of the triangles meet are called nodes, and each triangle formed by three nodes and three sides is called an element. It is possible to use other shapes than triangles for the elements, however using the two dimensional simplex gives a method that does not require numerical integration and hence 
results in a considerable saving in computation time.

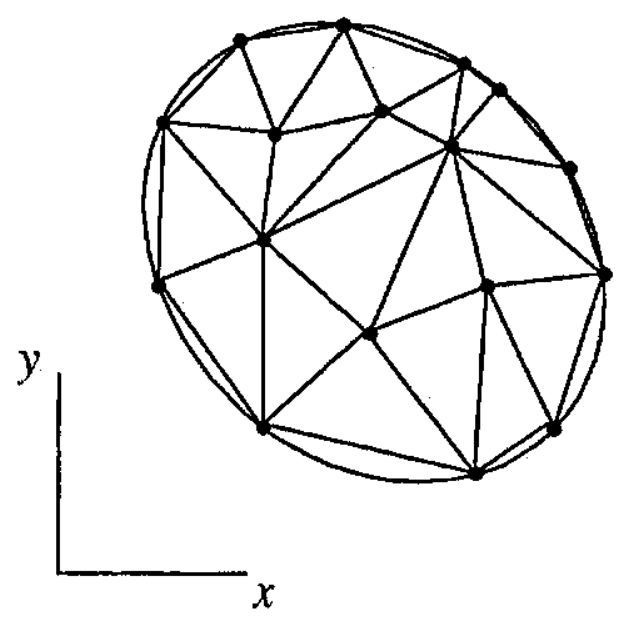

Figure 2.5: Triangular finite element discretisation of an arbitrary shape.

Each of the nodes at the vertices of the triangular elements has a unique global node number in the scheme of the entire mesh. Each element also has a local numbering system, from 1 to 3 , and by relating the local number to the global number of the three nodes for each element, the connectivity for the entire system can easily be stored. Figure 2.6 shows a typical element with local numbers circled within the element and global numbers in squares outside of the element. Local node 3 of this element is global node 10, which may be a vertex of one or many other elements.

With displacements allowed in the $x$ and $y$ directions, there are two degrees of freedom for each node and therefore 6 for each element. The element displacement vector, $\mathbf{q}=\left[q_{1}, q_{2}, \ldots, q_{6}\right]^{T}$, contains the displacements of each of its nodes in both the $x$ and $y$ directions. They are ordered such that local node $j$ has its displacement in the $x$ direction in $q_{2 j-1}$ and its displacement in the $y$ direction in $q_{2 j}$. The global displacement vector stores the displacements of all nodes and is arranged in a similar way to the local displacement 


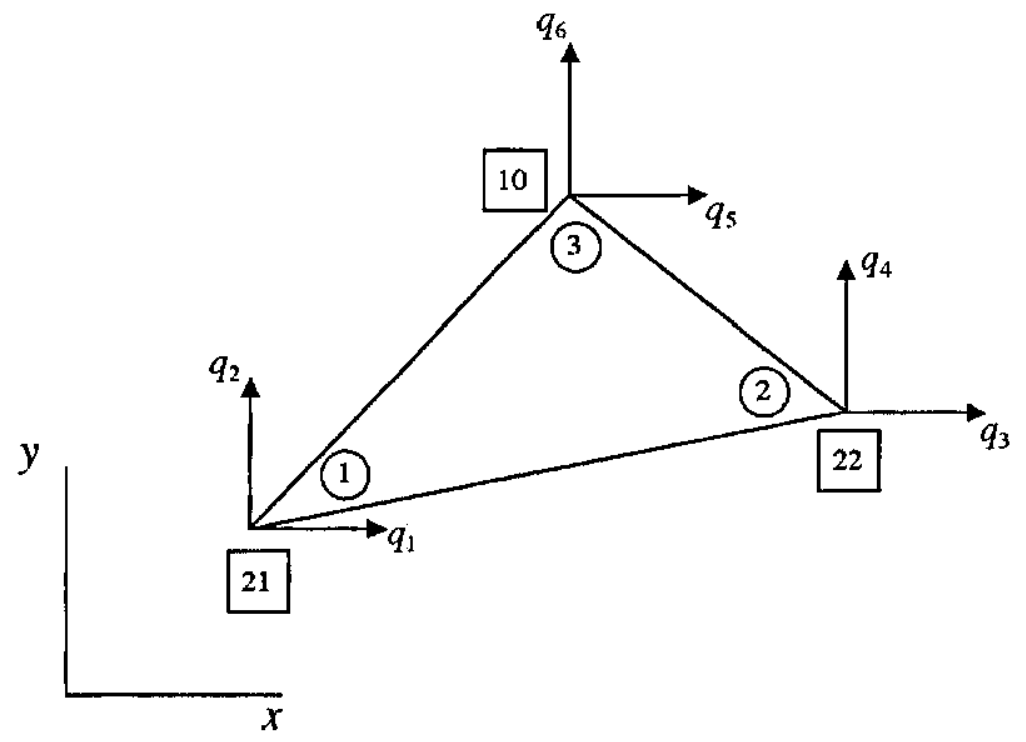

Figure 2.6: A typical element with local and global node number shown in circles and squares respectively.

vector such that global node $j$ has its displacement in the $x$ direction in $Q_{2 j-1}$ and its displacement in the $y$ direction in $Q_{2 j}$.

The stresses, $\boldsymbol{\sigma}$, and strains, $\boldsymbol{\epsilon}$, are related by $\boldsymbol{\sigma}=\mathbf{D} \boldsymbol{\epsilon}$. When considering plane stress, the $(3 \times 3)$ D matrix is given in terms of the material's Young's modulus and Poisson's ratio, $E$ and $\nu$.

$$
\mathbf{D}=\frac{E}{1-\nu^{2}}\left[\begin{array}{ccc}
1 & \nu & 0 \\
\nu & 1 & 0 \\
0 & 0 & \frac{1-\nu}{2}
\end{array}\right]
$$

Displacements of a point within an element need to be described due to the displacements of the nodes of the element, this is achieved by the concept of shape functions and interpolation. For the constant strain triangle, linear shape functions are applied to the element, $N_{1}, N_{2}$ and $N_{3}$ corresponding to nodes 1,2 and 3 . Shape function $N_{1}$ should be 1 at node 1 and linearly 
reduce to zero at nodes 2 and 3 . Similarly, shape functions $N_{2}$ and $N_{3}$ are 1 at nodes 2 and 3 respectively and drop to zero at the nodes on the opposite edges. Mapping a typical element to the standard element in figure 2.7 gives rise to simple shape functions in terms of the natural coordinates $\xi$ and $\eta$,

$$
N_{1}=\xi \quad N_{2}=\eta \quad N_{3}=1-\xi-\eta .
$$

The isoparametric representation for describing the $x$ and $y$ coordinates in terms of the nodal coordinates and the shape functions is given by

$$
\begin{aligned}
& x=N_{1} x_{1}+N_{2} x_{2}+N_{3} x_{3} \\
& y=N_{1} y_{1}+N_{2} y_{2}+N_{3} y_{3}
\end{aligned}
$$

where $x_{i}$ is the $x$ coordinate of local node $i$ and similarly for $y_{i}$, then defining $x_{i j}=x_{i}-x_{j}$ (the same definition is given for $y_{i j}$, but using $y$ coordinates), this can be written in terms of the natural coordinates as

$$
\begin{aligned}
& x=x_{13} \xi+x_{23} \eta+x_{3} \\
& y=y_{13} \xi+y_{23} \eta+y_{3}
\end{aligned}
$$

and similarly for the displacement vector $\left(\mathbf{u}=[u, v]^{T}\right)$

$$
\begin{aligned}
& u=u_{13} \xi+u_{23} \eta+u_{3} \\
& v=v_{13} \xi+v_{23} \eta+v_{3}
\end{aligned}
$$

In each element the strain is related to the displacements by $\epsilon=$ Bq. In the $2 \mathrm{D}$ case, the matrix $\mathbf{B}$ is calculated as

$$
\mathbf{B}=\frac{1}{\operatorname{det} \mathbf{J}}\left[\begin{array}{cccccc}
y_{23} & 0 & y_{31} & 0 & y_{12} & 0 \\
0 & x_{32} & 0 & x_{13} & 0 & x_{21} \\
x_{32} & y_{23} & x_{13} & y_{31} & x_{21} & y_{12}
\end{array}\right]
$$

where $\operatorname{det} \mathbf{J}=x_{13} y_{23}-x_{23} y_{13}$. 


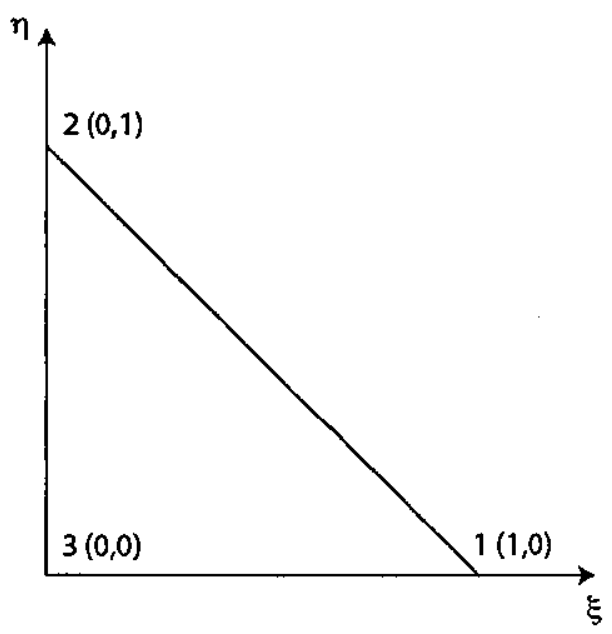

Figure 2.7: Each element is mapped to this standard element for simplicity of the shape functions in terms of the natural coordinates $\xi$ and $\eta$.

Using the potential energy approach, the element strain energy, $U_{e}$, is found to be

$$
U_{e}=\frac{1}{2} \mathrm{q}^{T} t_{e} A_{e} \mathrm{~B}^{T} \mathbf{D B q}
$$

where $A_{e}\left(=\frac{1}{2}|\operatorname{det} \mathbf{J}|\right)$ is the element area and $t_{e}$ is the constant element thickness, or

$$
U_{e}=\frac{1}{2} \mathbf{q}^{T} \mathbf{k}^{e} \mathbf{q}
$$

where $\mathbf{k}^{e}$ is the element stiffness matrix given by

$$
\mathbf{k}^{e}=t_{e} A_{e} \mathbf{B}^{T} \mathrm{DB} .
$$

A global stiffness matrix, $\mathbf{K}$, may be constructed from all of the element stiffness matrices such that the total system energy is given by

$$
U_{t}=\sum_{e} \frac{1}{2} \mathbf{q}^{T} \mathbf{k}^{e} \mathbf{q}=\frac{1}{2} \mathbf{Q}^{T} \mathbf{K} \mathbf{Q} .
$$

This global stiffness matrix is square symmetric and usually sparse or banded due to the fact that the stiffness value $\mathbf{K}_{\mathbf{i j}}$ is zero when the degrees of freedom $i$ and $j$ are not connected through an element. 
The nodal forces, $\mathbf{F}$, are found from the first derivative of the total energy in equation 2.47 with respect to nodal displacements, giving the relationship between displacements and forces,

$$
\mathbf{K Q}=\mathbf{F}
$$

where $\mathbf{F}$ stores the nodal forces such that the force on node $j$ in the $x$ direction is in $\mathbf{F}_{2 j-1}$ and in the $y$ direction, $\mathbf{F}_{2 j}$.

\subsubsection{Three Dimensional Finite Elements for Stress Analysis}

For most realistic engineering stress problems a three dimensional (3D) solution is required. A $3 \mathrm{D}$ volume can be tessellated using tetrahedra. Each tetrahedron is called an element and the 4 vertices of the tetrahedron are called nodes. Each node of an element has a local node number within the element, 1-4, and a global node number in the scheme of the entire mesh. Figure 2.8 shows a typical tetrahedral element with nodes labelled locally. Any of the nodes in this element may also be vertices of one or more other elements.

The displacement vector is given by,

$$
\mathbf{u}=[u, v, w]^{T}
$$

where $u, v$, and $w$ are displacements in the $x, y$, and $z$ directions respectively. Stresses and strains are related by

$$
\sigma=\mathbf{D} \epsilon
$$

where

$$
\begin{aligned}
\boldsymbol{\sigma} & =\left[\sigma_{x}, \sigma_{y}, \sigma_{z}, \tau_{y z}, \tau_{x z}, \tau_{x y}\right]^{T} \\
\boldsymbol{\epsilon} & =\left[\epsilon_{x}, \epsilon_{y}, \epsilon_{z}, \gamma_{y z}, \gamma_{x z}, \gamma_{x y}\right]^{T}
\end{aligned}
$$




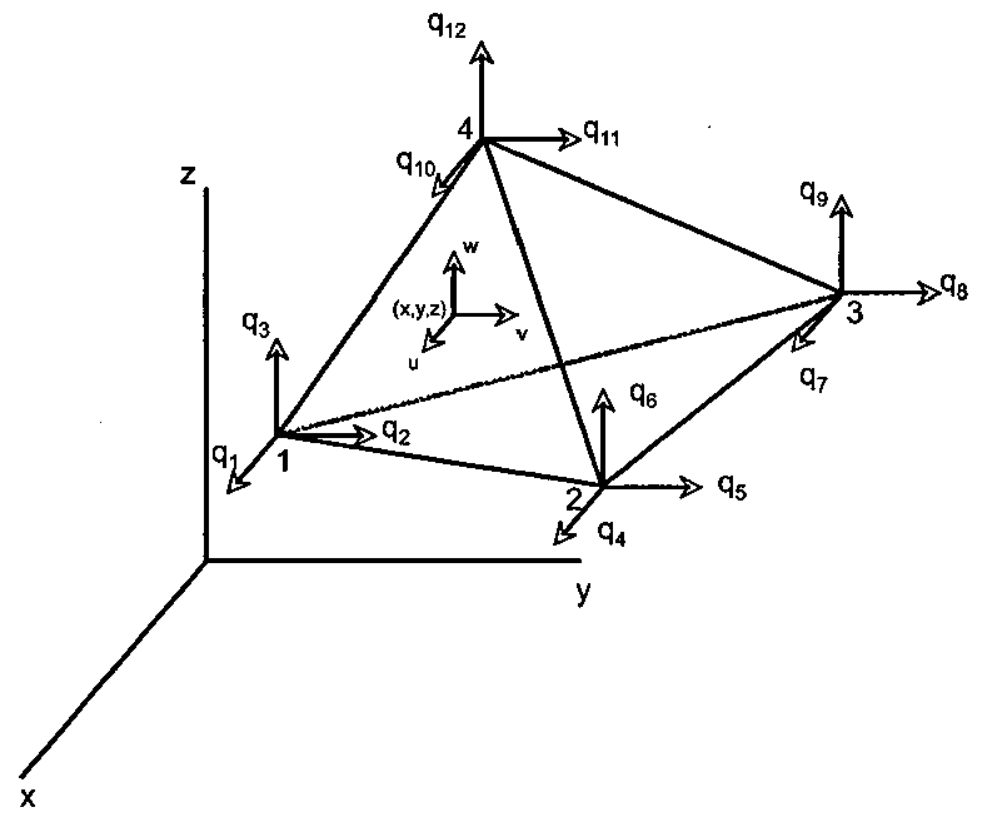

Figure 2.8: A typical tetrahedral element with local node numbers. Each node has three degrees of freedom as they can move in the $x, y$ and $z$ directions.

are the stresses and strains, and for isotropic materials, $\mathbf{D}$ is given by

$$
\mathbf{D}=\frac{E}{(1+\nu)(1-2 \nu)}\left[\begin{array}{cccccc}
1-\nu & \nu & \nu & 0 & 0 & 0 \\
\nu & 1-\nu & \nu & 0 & 0 & 0 \\
\nu & \nu & 1-\nu & 0 & 0 & 0 \\
0 & 0 & 0 & 0.5-\nu & 0 & 0 \\
0 & 0 & 0 & 0 & 0.5-\nu & 0 \\
0 & 0 & 0 & 0 & 0 & 0.5-\nu
\end{array}\right]
$$

The strain-displacement relationships for linear elasticity are given by

$$
\boldsymbol{\epsilon}=\left[\frac{\partial u}{\partial x}, \frac{\partial v}{\partial y}, \frac{\partial w}{\partial z}, \frac{\partial v}{\partial z}+\frac{\partial w}{\partial y}, \frac{\partial u}{\partial z}+\frac{\partial w}{\partial x}, \frac{\partial u}{\partial y}+\frac{\partial v}{\partial x}\right]^{T}
$$

In figure 2.8, each local node $i$ is labelled with three degrees of freedom 
$q_{3 i-2}, q_{3 i-1}$ and $q_{3 i}$ as it is free to move in three dimensional space. The corresponding global node $I$ is assigned the three degrees of freedom $Q_{3 I-2}, Q_{3 I-1}$ and $Q_{3 I}$, so that the element and global displacement vectors are given by

$$
\begin{gathered}
\mathbf{q}=\left[q_{1}, q_{2}, q_{3}, \ldots, q_{12}\right]^{T} \\
\mathbf{Q}=\left[Q_{1}, Q_{2}, Q_{3}, \ldots, Q_{N}\right]^{T}
\end{gathered}
$$

where $N$ is three times the number of nodes in the system. Four linear shape functions are defined, $N_{1}, N_{2}, N_{3}$ and $N_{4}$, where $N_{i}$ is 1 at local node i and zero at the other 3 nodes of the element. For example $N_{1}$ has a value of 1 at node 1 and linearly decreases to a value of 0 at nodes 2,3 and 4 . Considering the standard element in figure 2.9 , the four shape functions for the tetrahedron can be defined in terms of the natural coordinates $\xi, \eta$ and $\zeta$ as

$$
N_{1}=\xi \quad N_{2}=\eta \quad N_{3}=\zeta \quad N_{4}=1-\xi-\eta-\zeta
$$

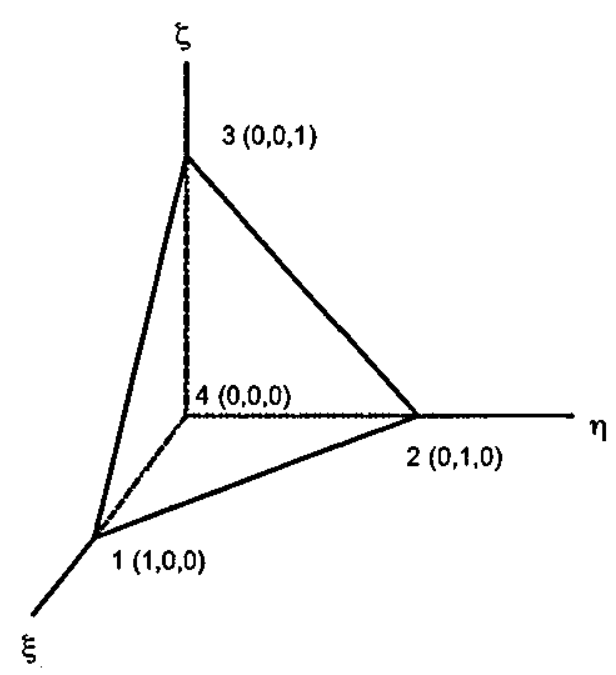

Figure 2.9: Each element is mapped to this standard element for simplicity of the shape functions in terms of the natural coordinates $\xi, \eta$ and $\zeta$. 
Defining $u_{i}, v_{i}$ and $w_{i}$ to be the displacements of node $i$ and $u_{i j}=u_{i}-u_{j}$, the displacements of an arbitrary point $(x, y, z)$ within the element can then be written in terms of the natural coordinates and nodal displacements as

$$
\begin{aligned}
u & =u_{4}+u_{14} \xi+u_{24} \eta+u_{34} \zeta \\
v & =v_{4}+v_{14} \xi+v_{24} \eta+v_{34} \zeta \\
w & =w_{4}+w_{14} \xi+w_{24} \eta+w_{34} \zeta .
\end{aligned}
$$

The coordinates of this point may be found similarly from the isoparametric transformation

$$
\begin{aligned}
& x=x_{4}+x_{14} \xi+x_{24} \eta+x_{34} \zeta \\
& y=y_{4}+y_{14} \xi+y_{24} \eta+y_{34} \zeta \\
& z=z_{4}+z_{14} \xi+z_{24} \eta+z_{34} \zeta
\end{aligned}
$$

The Jacobian of this transformation is given by

$$
\mathbf{J}=\left[\begin{array}{lll}
\frac{\partial x}{\partial \xi} & \frac{\partial y}{\partial \xi} & \frac{\partial z}{\partial \xi} \\
\frac{\partial x}{\partial \eta} & \frac{\partial y}{\partial \eta} & \frac{\partial z}{\partial \eta} \\
\frac{\partial x}{\partial \zeta} & \frac{\partial y}{\partial \zeta} & \frac{\partial z}{\partial \zeta}
\end{array}\right]=\left[\begin{array}{lll}
x_{14} & y_{14} & z_{14} \\
x_{24} & y_{24} & z_{24} \\
x_{34} & y_{34} & z_{34}
\end{array}\right]
$$

and performing a triple integral over the element, the volume of the element, $V_{e}$ is given by

$$
V_{e}=\frac{1}{6}|\operatorname{det} \mathbf{J}|
$$

where

$$
|\operatorname{det} \mathbf{J}|=x_{14}\left(y_{24} z_{34}-y_{34} z_{24}\right)+y_{14}\left(z_{24} x_{34}-z_{34} x_{24}\right)+z_{14}\left(x_{24} y_{34}-x_{34} y_{24}\right)
$$

Partial derivatives of $u$ with respect to $x, y$ and $z$ are related to $\xi, \eta$ and $\zeta$ 
derivatives by the following relationship,

$$
\left\{\begin{array}{c}
\frac{\partial u}{\partial x} \\
\frac{\partial u}{\partial y} \\
\frac{\partial u}{\partial z}
\end{array}\right\}=\mathbf{A}\left\{\begin{array}{c}
\frac{\partial u}{\partial \xi} \\
\frac{\partial u}{\partial \eta} \\
\frac{\partial u}{\partial \zeta}
\end{array}\right\}
$$

similarly for $v$ and $w$. This is the inverse relation to the transformation with Jacobian $\mathbf{J}$, hence

$$
\mathbf{A}=\mathbf{J}^{-1}=\frac{1}{\operatorname{det} \mathbf{J}}\left[\begin{array}{ccc}
y_{24} z_{34}-y_{34} z_{24} & y_{34} z_{14}-y_{14} z_{34} & y_{14} z_{24}-y_{24} z_{14} \\
z_{24} x_{34}-z_{34} x_{24} & z_{34} x_{14}-z_{14} x_{34} & z_{14} x_{24}-z_{24} x_{14} \\
x_{24} y_{34}-x_{34} y_{24} & x_{34} y_{14}-x_{14} y_{34} & x_{14} y_{24}-x_{24} y_{14}
\end{array}\right]
$$

By considering the strain displacement relationships in equation 2.54 , the assumed displacement field in equation 2.58 and the relation between derivatives in equation 2.63 , a relationship between strain and nodal displacements in obtained,

$$
\epsilon=\mathrm{Bq}
$$

where $\mathbf{B}$ is the following matrix made up of the elements of matrix $\mathbf{A}$.

$$
\mathbf{B}=\left[\begin{array}{cccccccccccc}
A_{11} & 0 & 0 & A_{12} & 0 & 0 & A_{13} & 0 & 0 & -\tilde{A}_{1} & 0 & 0 \\
0 & A_{21} & 0 & 0 & A_{22} & 0 & 0 & A_{23} & 0 & 0 & -\tilde{A}_{2} & 0 \\
0 & 0 & A_{31} & 0 & 0 & A_{32} & 0 & 0 & A_{33} & 0 & 0 & -\tilde{A}_{3} \\
0 & A_{31} & A_{21} & 0 & A_{32} & A_{22} & 0 & A_{33} & A_{23} & 0 & -\tilde{A}_{3} & -\tilde{A}_{2} \\
A_{31} & 0 & A_{11} & A_{32} & 0 & A_{12} & A_{33} & 0 & A_{13} & -\tilde{A}_{3} & 0 & -\tilde{A}_{1} \\
A_{21} & A_{11} & 0 & A_{22} & A_{12} & 0 & A_{23} & A_{13} & 0 & -\tilde{A}_{2} & -\tilde{A}_{1} & 0
\end{array}\right]
$$

Note that $\tilde{A}_{i}=A_{i 1}+A_{i 2}+A_{i 3}$.

Using the potential energy approach, the element strain energy, $U_{e}$, is found to be

$$
U_{e}=\frac{1}{2} \mathbf{q}^{T} V e \mathbf{B}^{T} \mathbf{D B} \mathbf{q}
$$


or

$$
U_{e}=\frac{1}{2} \mathbf{q}^{T} \mathbf{k}^{e} \mathbf{q}
$$

where $\mathbf{k}^{e}$ is the element stiffness matrix given by

$$
\mathbf{k}^{e}=V_{e} \mathbf{B}^{T} \mathrm{DB} .
$$

As in the 2D case in section 2.3.1, a global stiffness matrix, $\mathbf{K}$, may be constructed from all of the element stiffness matrices such that the total system energy is given by

$$
U_{t}=\sum_{e} \frac{1}{2} \mathbf{q}^{T} \mathbf{k}^{e} \mathbf{q}=\frac{1}{2} \mathbf{Q}^{T} \mathbf{K Q} .
$$

The nodal forces, $\mathbf{F}$, are found from the first derivative of the total energy in equation 2.70 with respect to nodal displacements, giving the relationship between displacements and forces,

$$
\mathbf{K Q}=\mathbf{F}
$$

where $\mathbf{F}$ stores the nodal forces such that the force on node $j$ in the $x$ direction is in $\mathbf{F}_{3 j-2}$, in the $y$ direction, $\mathbf{F}_{3 j-1}$ and in the $z$ direction, $\mathbf{F}_{3 j}$.

\subsubsection{Boeing-Harwell Packed Matrix Format}

The 2D and 3D finite element schemes described in sections 2.3.1 and 2.3.2 produce a set of linear simultaneous equations and once the global stiffness matrix and nodal forces have been constructed, these equations can be solved using a linear algebra routine to find the nodal displacements. However, with most real world problems, there are usually many elements and nodes, and because the stiffness matrix is square the system becomes large to store and to solve, due to limitations of memory and computation time. 
A method for packing sparse matrices known as the Boeing-Harwell format [19], allows sparse matrices to be stored without the many zero elements. The advantage of this method is not only that it saves large amounts of memory by ignoring the zeros, but also routines have been developed to work on matrices in this format, including the solution of simultaneous equations as an order $N$ process rather than order $N^{2}$, hence considerably reducing the time taken to perform the calculation.

The packing method works by replacing the matrix with three vectors. The first vector (VALUES in the example below) stores the nonzero matrix entries, with entries from the same column grouped together in the vector. The second vector (ROWIND in the example) contains the row index in the original matrix of the corresponding element in the VALUES vector. The number of elements in the ROWIND and VALUES vectors is equal to the number of nonzero entries in the original matrix. The final vector (COLPTR) stores pointers to the first element in the ROWIND and VALUES vectors of each column. For an $n \times n$ matrix, the COLPTR vector has $n+1$ entries, the last of which should point to one entry after the end of the ROWIND and VALUES vectors. The scheme is illustrated with the following simple example.

$$
\left(\begin{array}{ccccc}
1 . & -3 . & 0 & -1 . & 0 \\
0 & 0 & -2 . & 0 & 3 . \\
2 . & 0 & 0 & 0 & 0 \\
0 & 4 . & 0 & -4 . & 0 \\
5 . & 0 & -5 . & 0 & 6 .
\end{array}\right)
$$

The $5 \times 5$ matrix, 2.72 , is packed and stored in the three vectors displayed in table 2.1. The values from column $n$ are stored in the VALUES vector from elements $\operatorname{COLPTR}(n)$ to $\operatorname{COLPTR}(n+1)-1$. 
Chapter 2: Molecular Dynamics and Finite Elements Methodology

\begin{tabular}{l|ccccccccccc}
\hline Subscripts & 1 & 2 & 3 & 4 & 5 & 6 & 7 & 8 & 9 & 10 & 11 \\
\hline COLPTR & 1 & 4 & 6 & 8 & 10 & 12 & & & & & \\
ROWIND & 1 & 3 & 5 & 1 & 4 & 2 & 5 & 1 & 4 & 2 & 5 \\
VALUES & 1. & 2. & 5. & -3. & 4. & -2. & -5. & -1. & -4. & 3. & 6. \\
\hline
\end{tabular}

Table 2.1: Boeing-Harwell Packed Format

To retrieve the value of matrix element $(i, j)$, ROWIND values are checked from index $\operatorname{COLPTR}(j)$ to $\operatorname{COLPTR}(j+1)-1$. If none of these values are equal to $i$, then element $(i, j)$ is 0 . If the ROWIND value is equal to $i$, then the value of element $(i, j)$ is the value in the corresponding VALUES vector. 


\section{Chapter 3}

\section{Nanoindentation and Modelling Nanoindentation}

\subsection{Experimental Indentation}

Nanoindentation is a widely used technique for investigating mechanical properties of materials on the nano scale. In an indentation test, a hard indenter is forced into a test sample or substrate material. The increasing force applied to the tip during the experiment and the resulting depth of indentation are recorded and used to measure properties such as the hardness and elastic modulus of the material. A typical force-depth curve is shown in figure 3.1 .

The force applied to the indenter is increased with time up to a predetermined maximum, $P_{\max }$, where the maximum indentation depth is defined as $h_{\max }$. Oliver and Pharr [20] suggested that the contact depth, $h_{c}$ at the maximum depth is not equal to the maximum indentation depth due to the elastic deflection of the substrate surface. The amount of deflection is not the same for different shape tips and also depends on material properties of the 


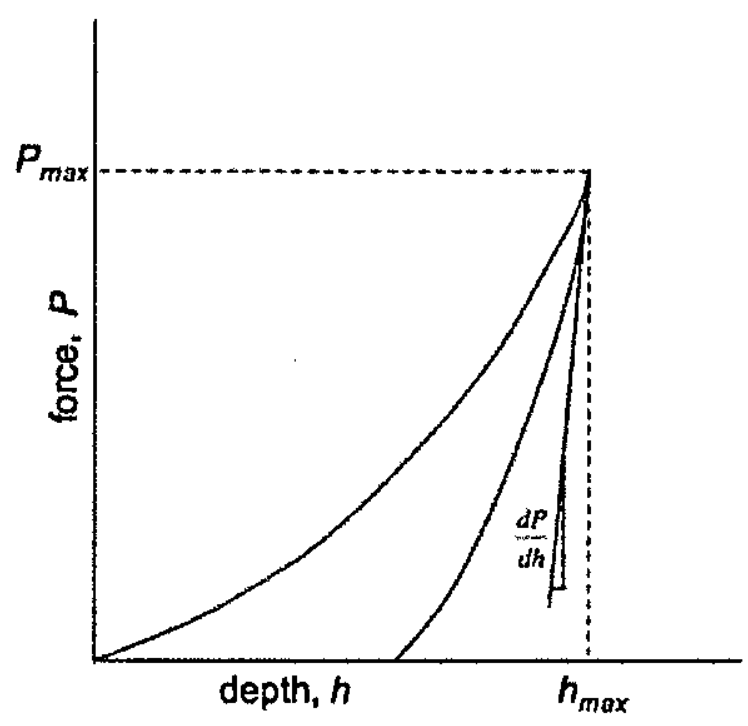

Figure 3.1: A typical force-depth curve from a nanoindentation experiment. The force on the indenter increases with depth to a maximum force of $P_{\max }$. The indenter is then held fixed for a holding period before being retracted. substrate such as stiffness. From the analytical solution for the shape of the substrate outside the contact area [21], the contact depth for a paraboloidal indenter is determined by

$$
h_{c}=h_{\max }-\frac{3}{4} \frac{P_{\max }}{S}
$$

where $S$ is the contact stiffness, defined as the slope of the unloading curve at the maximum depth, $S=\frac{d P}{d h}$. Then the corresponding cross-sectional area, $A_{c}$, of the indent may be obtained by the geometry of the indenter and the contact depth, i.e. $A_{c}=A_{c}\left(h_{c}\right)$. With this cross-sectional contact area known, the nanohardness, or contact pressure of the sample, defined as the ratio of the normal load to the contact area, can be calculated by

$$
H_{N}=\frac{P_{\max }}{A_{c}\left(h_{c}\right)}
$$


Oliver and Pharr also describe how the reduced Young's modulus $E_{r}$ of the indenter-sample system is calculated from the force-depth curve according to the formula

$$
E_{r}=\frac{1}{2} \sqrt{\frac{\pi}{A_{c}\left(h_{c}\right)}} \frac{d P}{d h} .
$$

The Young's modulus of the sample can then be obtained according to the equation

$$
\frac{1}{E_{\tau}}=\frac{1-\nu_{s}^{2}}{E_{s}}+\frac{1-\nu_{t}^{2}}{E_{t}}
$$

where $E_{s}$ and $\nu_{s}$ are the Young's modulus and Poisson's ratio of the substrate, and similarly for the indenter. For soft materials, the substrate's Young's modulus will hardly differ from $E_{r}$ as the indenter hardness is far higher than that of the substrate. When considering superhard materials and a diamond indenter, $E_{s} \approx E_{t}$ and Poisson's ratio is very small, thus $E_{s}$ is approximately double $E_{r}$.

For indentations experiencing only elastic deformation, the unloading curve follows the loading curve. Based on this principle, Dub [22] reported a new approach to determine contact pressure and Young's modulus through Hertzian elasticity theory. However, the method will not be used to calculate mechanical properties from the results in section 3.3 below as it assumes a spherical indenter and in this work a pyramidal indenter is used.

\subsubsection{AFM Cantilever Indentation}

The atomic force microscope (AFM) was originally designed to give visual representation of materials at the micron length scale. A sharp tip at the end of an elastically deformable cantilever is brought close enough to the material that atomic forces between the tip and the surface are experienced. A laser directed at the end of the cantilever measures its deflection, indicating the 
local sample height. A schematic representation of this is shown in figure 3.2 .

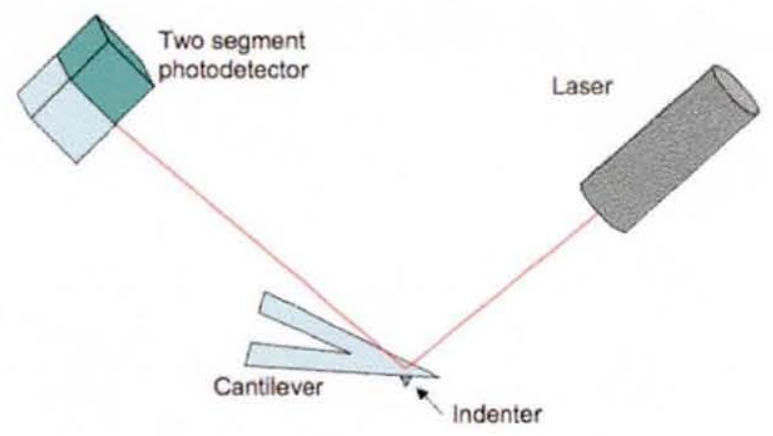

Figure 3.2: A schematic of the AFM cantilever system. The laser and photodetector are used to measure the deflection of the end of the cantilever.

Using a hard (generally diamond or SiN) indenter as the tip on the cantilever, the applied load is increased so that the indenter is forced into the substrate. The vertical cantilever deflection data obtained during the indentation along with the applied load data, is used to calculate mechanical properties of the substrate as described in section 3.1 above. Figure 3.3 shows a picture taken of a cantilever used in an AFM. Images at three different resolutions are displayed to give an accurate description of the cantilever-tip system.

The model of nanoindentation described in this work is based on the AFM cantilever method, but it should be noted that this is not the only method for nanoindentation. Transducer based systems such as the vertical force transducer are also common in experimental work. 


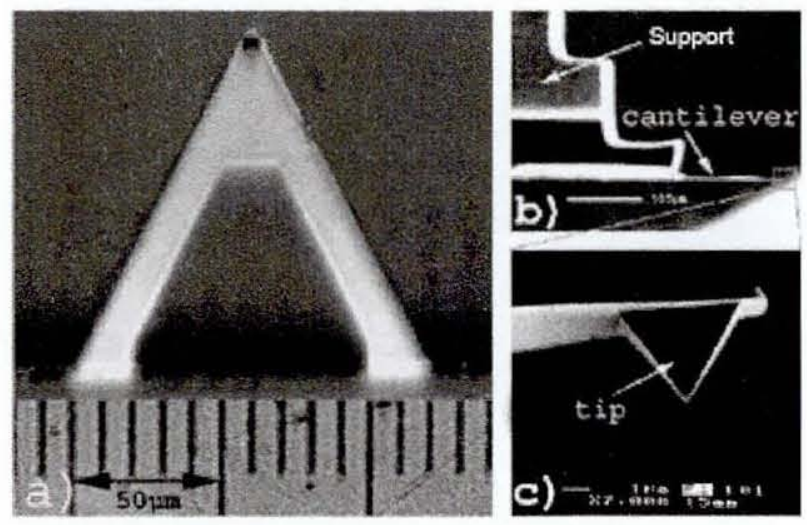

Figure 3.3: A pyramidal cantilever used in an AFM, picture courtesy of Professor A. Richter, University of Applied Sciences, Wildau. (a) shows a bottom-up view of the cantilever with the tip at the vertex. (b) shows the cantilever attached to the movable support in the AFM. (c) shows a further magnification of the cube corner tip.

\subsection{Atomistic Modelling of Nanoindentation}

Results from atomistic scale simulations which model nanoindentation can be used to compare to experimental results. These comparisons help to give a better understanding of the phenomena seen in experiments on the nano scale, and help to explain or interpret the experimental results.

The model used in the simulations in section 3.3 is called the 'spring model'. This model is designed to represent a system resembling indentations with the AFM cantilever described in section 3.1.1. Supports and springs are used to control the motion of the indenter, which is forced into the material under investigation. These springs model the elastic bending of the cantilever in the AFM which produces the force on the indenter in the experiment. 


\subsubsection{The Spring Model for Indentation}

The spring model used to simulate nanoindentation gets its name from the springs used to control the indentation process. A schematic representation of the system is shown in figure 3.4 where a diamond indenter is positioned above a substrate of the material under investigation and is attached to three supports by connecting springs. Displacement of the supports will result in

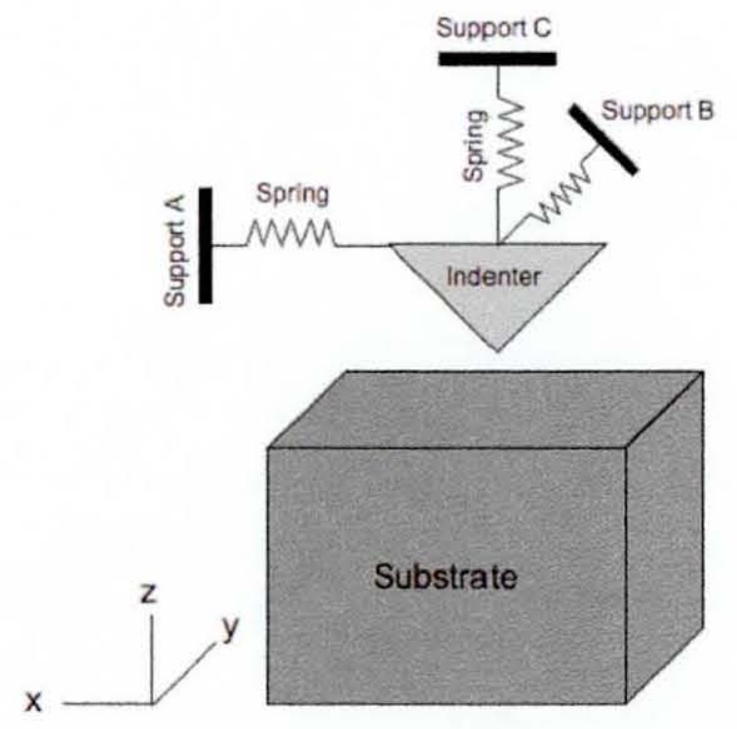

Figure 3.4: A schematic representation of the spring model for indentation. The indenter is attached to three supports via springs in the $x, y$ and $z$ directions. The displacement of the supports cause the motion of the indenter. the compression or extension of the springs, resulting in an applied force to the indenter. In an indentation simulation, the supports labelled A and B are fixed in the horizontal $x$ and $y$ directions, allowing the indenter to move laterally during the indentation. The vertical support labelled $\mathrm{C}$ is displaced as a function of time, $w_{C}(t)$. A typical displacement function for indentation is shown in figure 3.5, where the slope during indentation and extraction is 
the velocity applied to the support.

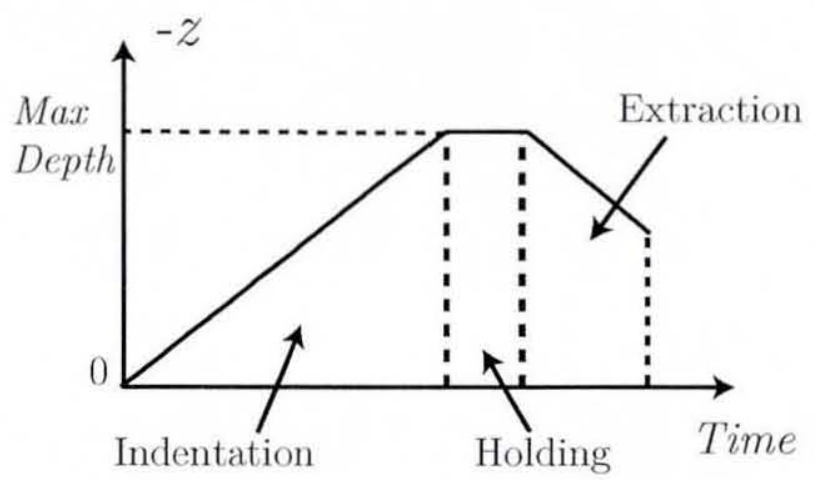

Figure 3.5: Typical vertical motion of the indenter during the simulation. The displacement does not always need to return to zero due to plastic deformation. The simulation may be terminated when there is no resultant force on the indenter.

The atoms on the top layer of the indenter are treated as a combined mass of material that move together. Defining the displacements of this mass of atoms to be $u_{a}, v_{a}$ and $w_{a}$, and the forces due to the other indenter atoms to be $F_{x}, F_{y}$ and $F_{z}$ in the $x, y$ and $z$ directions respectively, the equations of motion for the top of the indenter are given by

$$
\begin{aligned}
m a_{x}(t) & =F_{x}(t)+k_{x} u_{a}(t) \\
m a_{y}(t) & =F_{y}(t)+k_{y} v_{a}(t) \\
m a_{z}(t) & =F_{z}(t)+k_{z}\left(w_{a}(t)-w_{C}(t)\right)
\end{aligned}
$$

where $m$ is the mass of the combined atoms, $a_{x}, a_{y}$ and $a_{z}$ are the accelerations of the mass of atoms, and $k_{x}, k_{y}$ and $k_{z}$ are the spring constants. 


\subsubsection{Cube Corner Indenter Specification}

The indenter is modelled as a triangular based pyramid with a $90^{\circ}$ opening angle from a cubic diamond lattice with $\{100\}$ surfaces, which is cut along the diagonal (111) plane as shown in figure 3.6.

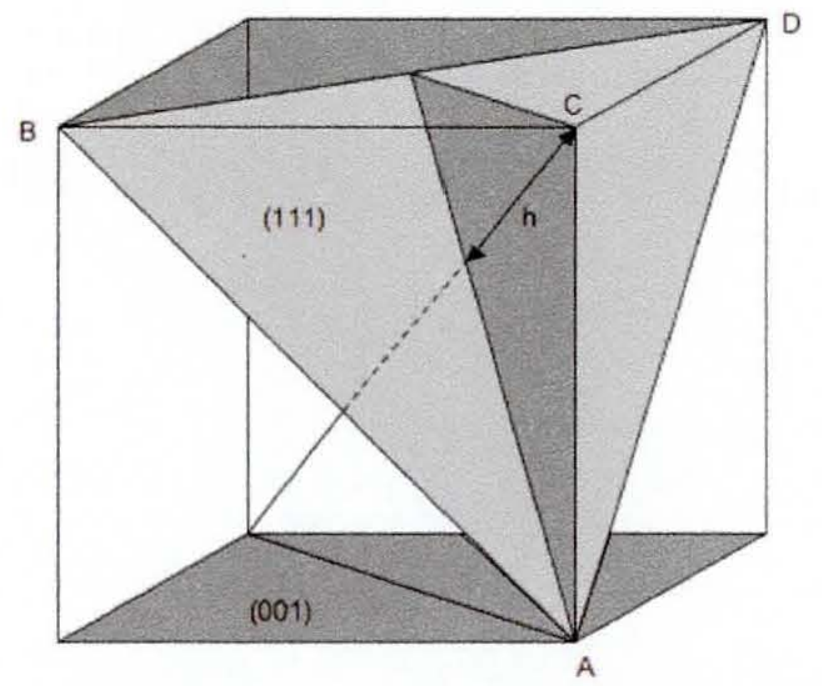

Figure 3.6: A schematic of the crystal structure of the indenter. A corner is cut along the (111) plane from a cubic diamond lattice with $\{100\}$ surfaces, resulting in an indenter with a $90^{\circ}$ opening angle.

The indenter is orientated such that the (111) planes are parallel to the substrate surface with the vertex formed by three intersecting $\{100\}$ faces pointing towards the surface. These $\{100\}$ surfaces are dimer reconstructed to give a more stable structure than the truncated crystal. Experimentally the tip is never atomically sharp, and so to model the experiment more closely a rounding of the tip is introduced by truncating a number of atoms from the vertex resulting in an appropriate curvature, this can be seen in figure 3.7. 


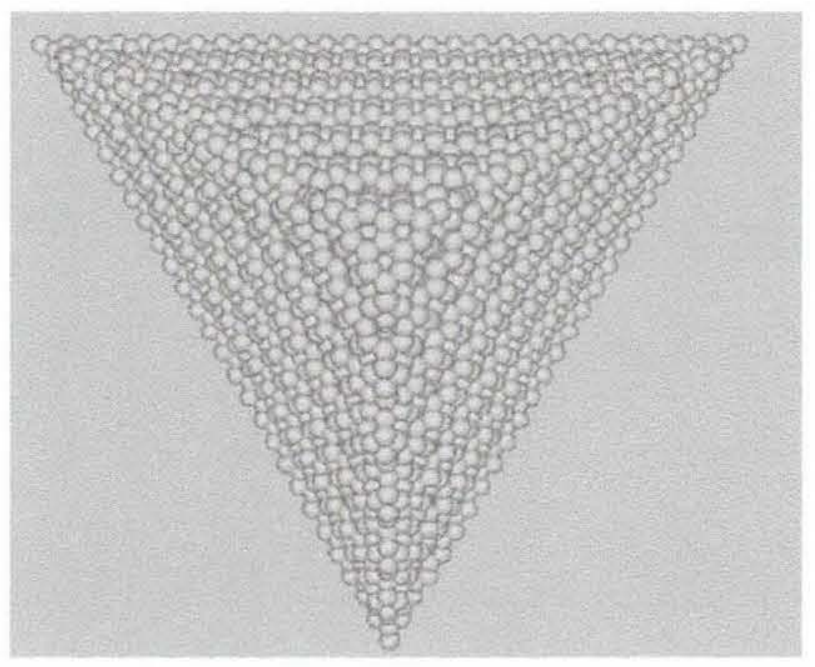

Figure 3.7: A plan view of an indenter with a truncated tip and dimer reconstructed $\{100\}$ surfaces.

\subsubsection{Finite Temperature Control}

The atoms in the substrate lattice and in the indenter begin in their equilibrium positions with zero velocity, that is to say the system is in its minimum energy configuration. When the simulation begins, forces on all atoms are balanced and hence, due to Newton's first law, the particles remain at rest. As temperature is proportional to the square of the velocities of the atoms, when all atoms are at rest the system is at absolute zero, or $0 \mathrm{~K}$.

In reality these experiments are generally carried out at room temperature (approximately $300 \mathrm{~K}$ ), where different results may be obtained than at absolute zero. Hence to model the experiment more closely a technique is implemented to thermalise the system before the indentation begins.

During indentation, the process generates local heating in the substrate which in reality would be dispersed into the far field. However, with only a relatively small number of atoms modelled $\left(O\left(10^{5}\right)\right)$, the lattice experiences 
artificially high temperatures. To control this heating, a low indentation speed is used $(\approx 10 \mathrm{~m} / \mathrm{s})$ and a thermostat is applied to the substrate which controls the temperature of the substrate during the simulation. Two commonly used thermostats are the Berendsen thermostat [23] and the NoseHoover thermostat $[24,25]$. The Nose-Hoover thermostat is more accurate but more complex than the Berendsen thermostat which is computationally much faster. The Nose-Hoover thermostat produces large oscillations in temperature before reaching the required target. This can cause problems when applying hydrogen termination to a substrate as the high temperatures can result in the hydrogen atoms leaving the surface.

\section{The Berendsen Thermostat}

One method of controlling the temperature of the system, both for thermalising at the start of the simulation and preventing artificial heating during indentation, is to apply the Berendsen thermostat. The method works by applying a velocity scaling to all or some of the atoms at each time step. The correction term, $\chi$, is calculated by comparing the average temperature of the lattice with the target temperature of the heat bath and the velocities of the atoms are then multiplied by $\chi$, given by

$$
\chi=\left[1+\frac{\delta t}{\tau}\left(\frac{T_{\text {bath }}}{T}-1\right)\right]^{\frac{1}{2}}
$$

where $\tau$ is the user defined time constant which controls the rate at which energy is added to or removed from the system, $T_{b a t h}$ is the target temperature, $\delta t$ is the time step of the simulation and $T$ is the average temperature of the lattice. A relationship between the kinetic energy of the particles, $U_{K E}$ and their average temperature is given by

$$
T=\frac{2 U_{K E}}{3 k_{B} n},
$$


where the Boltzman constant $k_{B}=8.617 \times 10^{-5} \mathrm{eV} \mathrm{K}^{-1}$, and $n$ is the number of particles. The kinetic energy of the $n$ particles is given by $U_{K E}=$ $\frac{1}{2} \sum_{i=1}^{n} m_{i} v_{i}^{2}$, where $m_{i}$ and $v_{i}$ are the mass and velocity of particle $i$ respectively.

\section{Boundary Conditions for Thermalised Systems}

For atomistic simulations of nanoindentation in this work, fixed boundary conditions (described in section 2.1.2) are applied to all boundaries in the lateral directions, and also on one boundary in the vertical direction at the bottom of the substrate. The atoms at top surface of the substrate are left to move freely as this is where the indentation takes place. The simulations in section 3.3 use the Berendsen thermostat to thermalise the atoms and to control temperature during the simulation. A layer of atoms within the fixed atoms are connected to a heat bath as shown in figure 3.8.

The indenter has a fixed layer of atoms at the top, and a layer of thermostat atoms directly below. Before the indentation process, the system is heated to the required temperature, first by considering that all thermostat atoms and free atoms are connected to the heat bath for an initial period to increase the temperature. Subsequently only thermostat atoms are connected to the heat bath until the target temperature is reached. The thermostat atoms remain connected to the heat bath during the indentation process to maintain a constant temperature. 

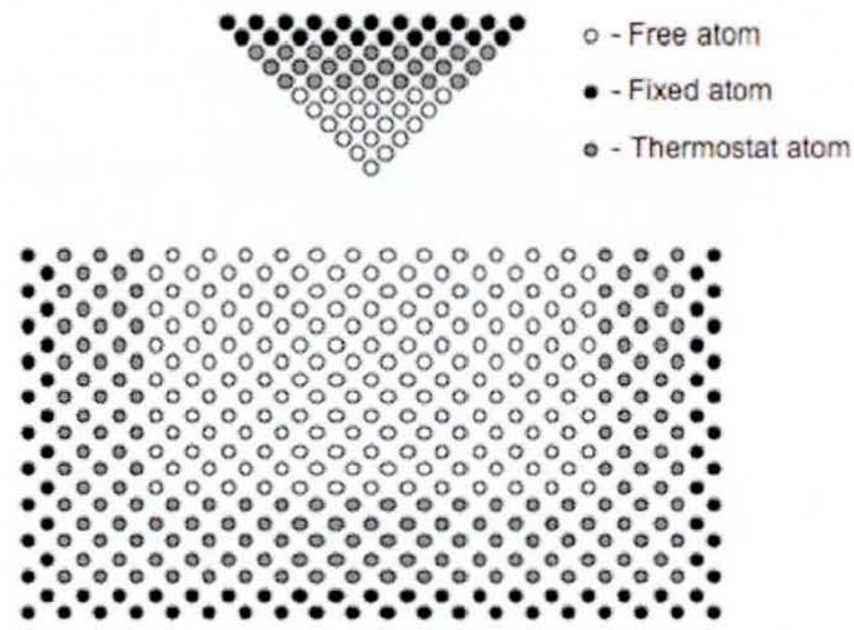

Figure 3.8: A schematic diagram of atom types for a thermalised system. Black atoms are fixed in position during the simulation. The grey atoms are connected to a heat bath which controls the system temperature during the simulation. The white atoms are free unconstrained atoms under the MD methodology.

\subsection{Simulation Results}

\subsubsection{Diamond Indentation Results}

A molecular dynamics simulation of nanoindentation of a diamond substrate was performed using a diamond cube corner indenter. The indenter was placed with its vertex $5 \AA$ above the centre of the unconstrained top surface of the lattice which was hydrogen terminated to reduce adhesion of the indenter and the substrate. The Brenner potential, described in section 2.2.4, was used to describe the interatomic interactions.

The indenter is modelled by 8,432 carbon atoms, the top 4 (111) planes of which were held fixed and connected to a spring with spring constant 352.4 
$\mathrm{N} / \mathrm{m}$, similar to that of a cantilever in an AFM. Other atoms within $22 \AA$ of the top plane were connected to a heat bath to control the temperature of the indenter during the indentation. The hydrogen terminated diamond substrate contained 291,232 atoms and had dimensions $145 \times 145 \times 85 \AA^{3}$. With the exception of the free top surface, atoms within $5 \AA$ of the edge of the substrate were held fixed, then other atoms within $25 \AA$ of the edge were connected to a heat bath to control the temperature of the substrate during the indentation. Before the indentation, the substrate and the indenter were both heated to $300 \mathrm{~K}$ by the methodology outlined in section 3.2.3. A prescribed velocity was given to the vertical support, applying a force to the top indenter atoms connected to the support via the vertical spring. The velocity applied in this work was $10 \mathrm{~m} / \mathrm{s}$ because of constraints on simulation time. This is faster than in the experiments, but is slow enough that local heating is controlled. The velocity of the support remains constant until it has moved a vertical distance of $68.4 \AA$ towards the substrate. The support is then held fixed for a holding period of $10 \mathrm{ps}$ at the maximum depth to allow relaxation before the support is retracted at the same rate as indentation until the indenter is completely removed from the substrate.

Although the vertical support moves a distance of $68.4 \AA$, the hardness of the substrate causes a considerable compression of the connecting spring and the top fixed layers of the indenter only move a distance of $27.6 \AA$ towards the surface. Moreover, there is a further compression of the indenter by about one third of its height, and hence the apex moves only $18.5 \AA$, an indentation depth of $13.5 \AA$ due to the initial gap between indenter and substrate. The extent of the indenter compression is shown in figure 3.9. The distortion of the indenter means that the contact area can not be calculated from its original geometry, and must be obtained by graphical means from 
the simulation results.

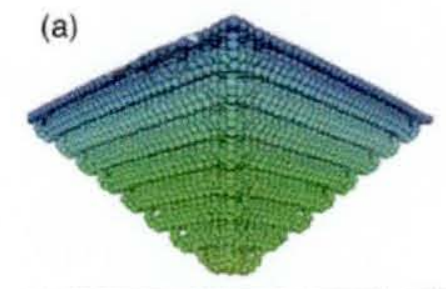

(c)

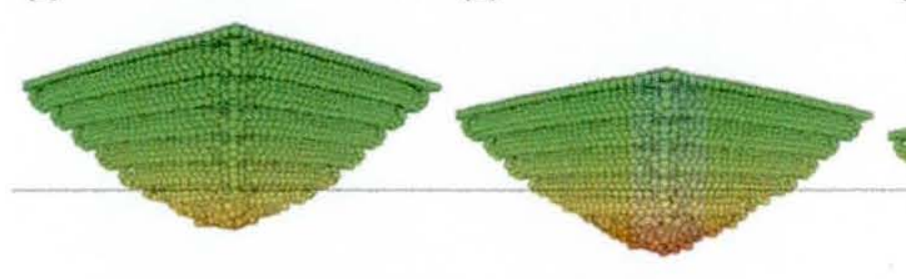

(b)

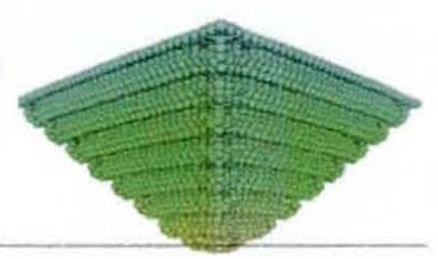

(d) (e)

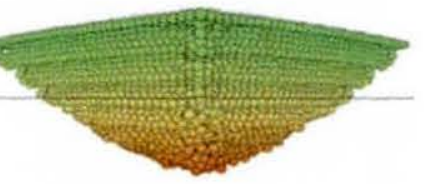

Figure 3.9: MD simulation of successive images of the cube-corner diamond indenter as it penetrates the diamond (100) surface showing the indenter compression. The horizontal line is the position of the surface. The images are given after every 100 ps of the indentation process.

The force-depth curve for the simulation is shown in figure 3.10. This curve shows that only elastic deformation occurs until the indenter is extracted from the substrate at the end of the simulation. From the images of the simulation in figure 3.12 , it can be seen that substrate atoms return to their original position, however there is some damage to the indenter where atoms have been left attached to the substrate surface. Although there is a layer of $\mathrm{H}$ atoms on the top surface of the substrate, the indenter consists purely of $\mathrm{C}$ atoms, and these experience strong attractive interactions with the $\mathrm{H}$ atoms of the substrate, hence causing indenter debris to be left on the surface.

Mechanical properties from the simulation are compared to experimental 


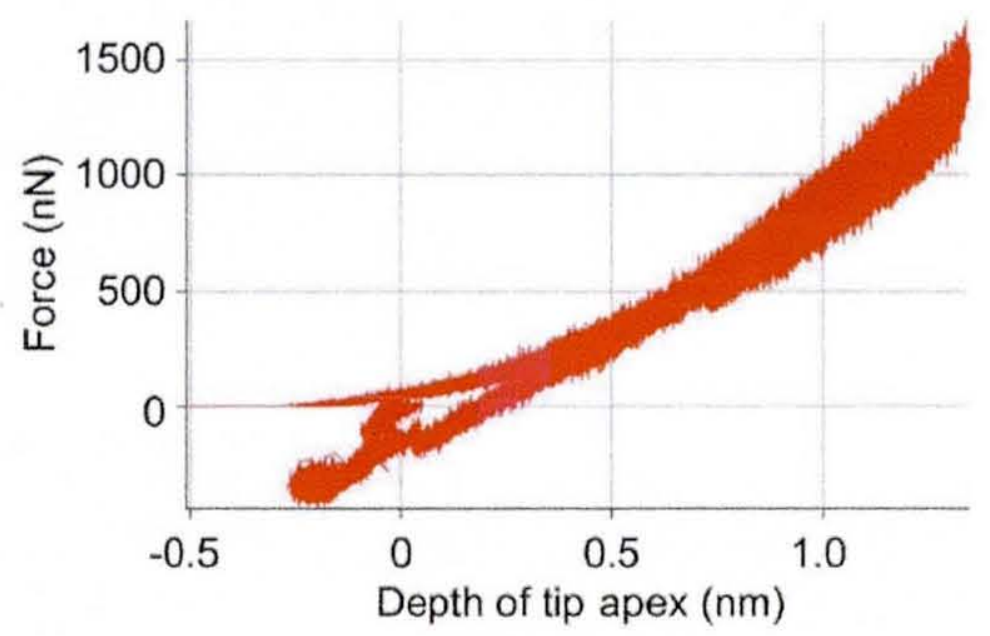

Figure 3.10: Force depth curve for the indentation of the cube corner indenter into H-terminated diamond (100). The curve profile shows only elastic deformation until the indenter is removed from the substrate.

values from the literature [26]. The contact area at the maximum depth is graphically determined to be $11.24 \times 10^{-18} \mathrm{~m}^{2}$, and the applied load at this depth is $1423 \mathrm{nN}$. Using equation 3.2 the contact pressure is calculated as $127 \mathrm{GPa}$, compared with the experimental value of $95 \mathrm{GPa}$. Equations 3.3 and 3.4 are used to calculate the Young's modulus of the diamond substrate as $E_{s}=940 \mathrm{GPa}$, also comparable with the experimental value of $912 \mathrm{GPa}$.

The curve in the force-depth graph follows the power law, $F \propto h^{m}$, with $m=1.6$ for this simulation. The curve is comparable to the experimental result for the indentation into diamond (100), even though the experimental indentation depth is far greater than in the simulation, see figure 3.11. In the experiment $m=1.6$ which is close to the theoretical value of 1.5 for a spherical indenter under elastic deformation [20].

The shallow indentation depth and purely elastic deformation of the sub- 


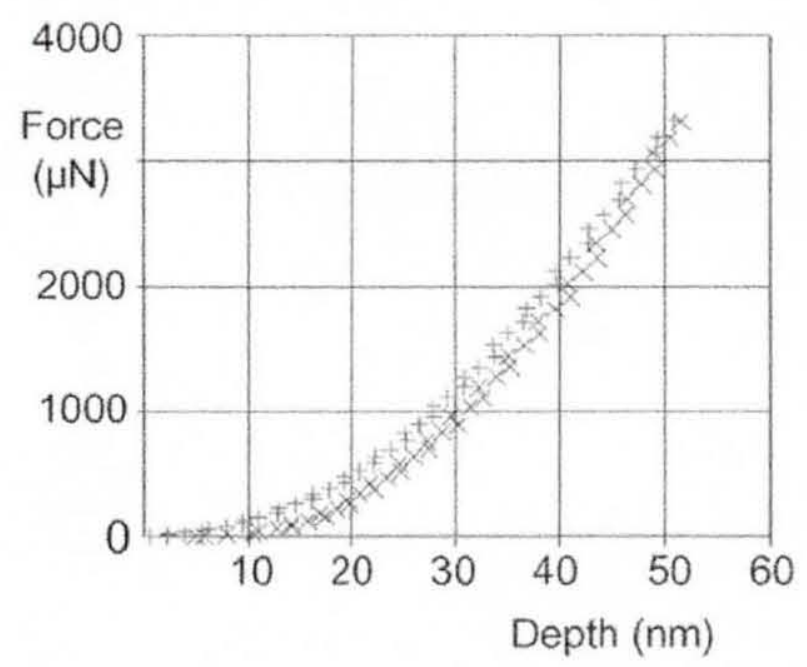

Figure 3.11: The experimental force-depth curve for indentation into diamond (100) [26].

strate gives results for contact pressure and Young's modulus in close agreement with experimental results. The hydrogen termination of the substrate did not completely remove indenter-substrate adhesion. A solution to this may be to hydrogen terminate the indenter surfaces as well as the substrate surface, although this has proved difficult as thermalisation has caused the hydrogen to leave the indenter at the edges. Another solution may be to neglect the attractive part of the potential between the indenter and the substrate, giving purely repulsive forces.

\subsubsection{Silicon Indentation Results}

A molecular dynamics simulation, modelling nanoindentation of a silicon substrate was performed. A diamond cube corner was used for the indenter and was initially positioned with its vertex $5 \AA$ above the hydrogen terminated (100) silicon surface, and the (111) planes parallel to the substrate surface. 


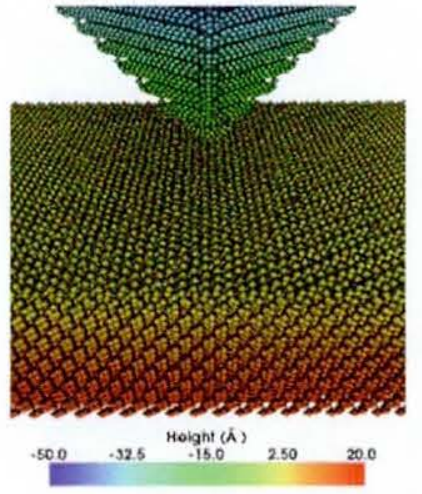

(a) $0 \mathrm{~ns}$

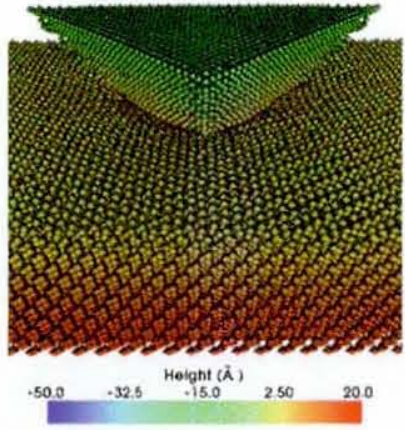

(b) $0.5 \mathrm{~ns}$

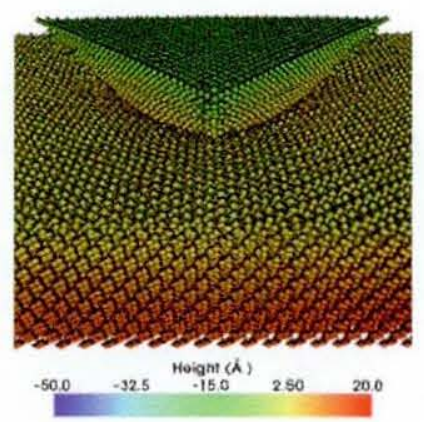

(c) $0.7 \mathrm{~ns}$

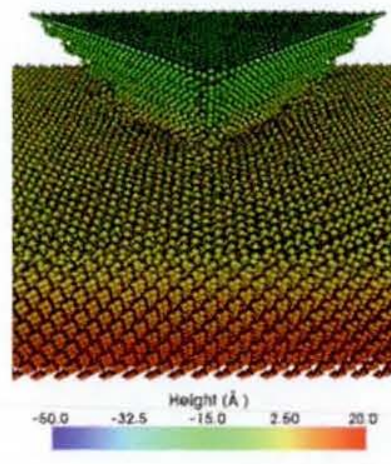

(d) $1.1 \mathrm{~ns}$

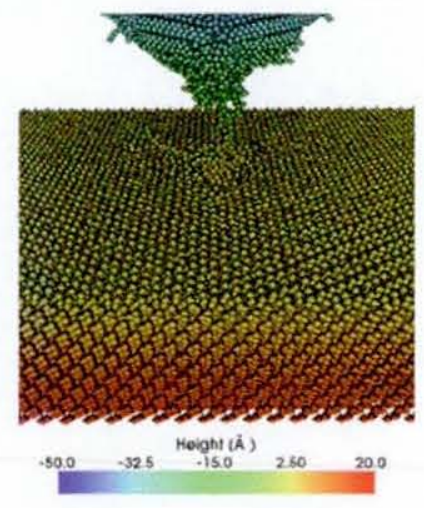

(e) $1.5 \mathrm{~ns}$

Figure 3.12: Images from various stages of the diamond (100) indentation. The maximum depth is reached after $0.7 \mathrm{~ns}$.

The Tersoff potential (see section 2.2.3) was used to describe interatomic interactions for the $\mathrm{Si}$ atoms in the substrate, and the Brenner potential (see section 2.2.4) was used to describe interactions between $\mathrm{C}$ atoms in the indenter.

The same indenter and spring were used as for the diamond indentation described in section 3.3.1, with 8,432 carbon atoms, the top 4 (111) planes held fixed and connected to a spring with spring constant $352.4 \mathrm{~N} / \mathrm{m}$, and non-fixed atoms within $22 \AA$ of the top of the indenter connected to 
a heat bath in order to control the temperature of the indenter during the simulation. The silicon substrate was hydrogen terminated at the top unconstrained surface to reduce adhesion with the indenter. The other surfaces have fixed atoms up to $5 \AA$ from the boundaries, and atoms connected to a heat bath for a further $20 \AA$ to control the temperature of the substrate during the simulation. The substrate contained 205,351 atoms and had dimensions $200 \times 200 \times 102 \AA^{3}$. Before the indentation, the substrate and the indenter were both heated to $300 \mathrm{~K}$ by the methodology outlined in section 3.2.3. A velocity of $10 \mathrm{~m} / \mathrm{s}$ was given to the vertical support, applying a force to the top indenter atoms connected to the support via the vertical spring. The velocity of the support remained constant until it had moved a vertical distance of $41.8 \AA$ towards the substrate. The support was then held fixed for a holding period of $10 \mathrm{ps}$ at the maximum depth to allow relaxation before the support is retracted at the same rate as indentation until the indenter is completely removed from the substrate. Due to the difference in hardness of the indenter and the substrate, the indenter compression was negligible. There was, however, a compression of the spring between the indenter and the support. Hence, of the $41.8 \AA$ displacement of the support, the indenter is displaced $30 \AA$, and due to the initial $5 \AA$ gap between indenter and substrate, the indentation depth $h_{\max }=25 \AA$.

A top-down view of the silicon surface is shown in figure 3.13. The image of the imprint at the maximum depth shows that the atoms at the tip apex have been displaced $25 \AA$, and also that there is very little elastic bending of the surface at the point of indentation. The image of the imprint after the indenter has been removed shows that the hole depth is around $15 \AA$, hence there is an elastic recovery of $10 \AA, 40 \%$ of the maximum indentation depth. 


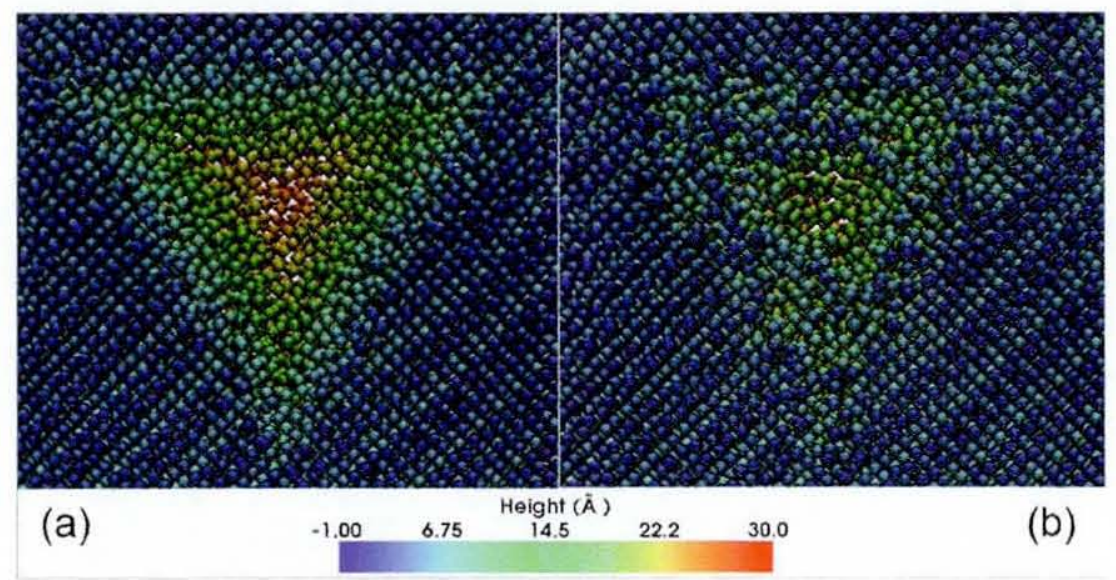

Figure 3.13: A top-down view of the indentation into Si (100): (a) at the maximum indentation depth of $25 \AA$. (b) after the indenter has been removed. This illustrates the elastic recovery of the substrate when the indenter is extracted.

Figure 3.14 shows the force-depth curve from the simulation. The large hysteresis in this curve shows that there is considerable plastic deformation of the substrate due to the indentation. The large negative force on the tip during extraction suggests that even with the hydrogen termination of the substrate, there is still strong adhesion between indenter and sample.

Mechanical properties from the simulation are compared to experimental values from the literature [27]. The contact area at the maximum depth is graphically determined to be $16.68 \times 10^{-18} \mathrm{~m}^{2}$, and the applied load at this depth is $400 \mathrm{nN}$. Using equation 3.2 the contact pressure is calculated as 24.1 $\mathrm{GPa}$, compared with the experimental value of $12 \mathrm{GPa}$, this is approximately double. Equation 3.3 is used to calculate the Young's modulus of the silicon substrate as $E_{s}=76 \mathrm{GPa}$. There is disagreement again with the experimental result of $140 \mathrm{GPa}$. These differences may be due to the indentation depth dependancy of contact pressure, or that fixed boundary conditions were ap- 


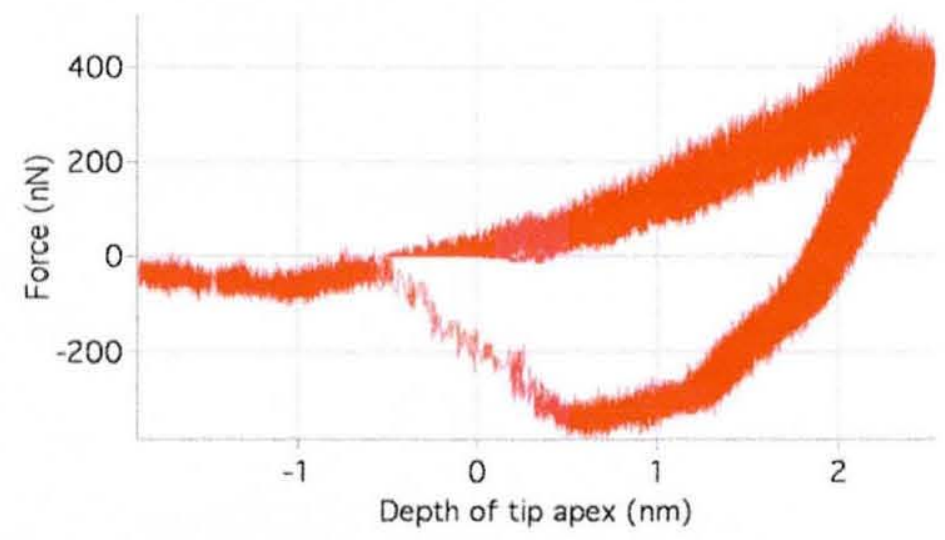

Figure 3.14: Force depth curve for the indentation of the cube corner indenter into H-terminated silicon (100). The curve profile shows extensive plastic deformation during indentation. A strong adhesion between indenter and sample is indicated by the negative force during unloading.

plied to the atomistic substrate, which produces an artificially high material hardness. The experimental indentation depth was about 300 times deeper than in the simulation.

\subsection{Conclusions}

At larger indentation depths, the fixed boundary conditions applied at the edge of the substrate have an effect on the material properties of hardness and Young's modulus, causing a disagreement with experimental results. A possible solution to the limitation of system size for nanoindentation simulations, is to introduce a hybrid multiscale model. These models are used to span the length scales and allow considerably larger volumes of material to be modelled, without the expense of an atomistic representation in the far field where continuum mechanics may be used to describe the material. By apply- 
ing continuum boundary conditions to a molecular dynamics simulation, the artificial fixed boundaries are removed from the edge of the atomistic region. It is hoped that this will provide a more physically accurate model, giving results in closer agreement with the experiment. 


\section{Chapter 4}

\section{Existing Multiscale Models}

This chapter will introduce multiscale modelling and give a review of some of the existing coupling techniques. A published topical review [28] analyses some of the existing multiscale models, outlining their methodology within a common framework in order to highlight the differences and advantages of the models. A detailed description of a selection of these models will be given in this chapter.

Linking atomistics to continuum models originated in the 1970's when work was done creating continuum elasticity boundary conditions for atomistic regions in an attempt to extend length scales [29, 30, 31]. Due to computational constraints, these system were limited to around only 1000 atoms. Sinclair's model [31] treated the continuum region analytically, using equilibrium solutions to modify the atomistic boundary during energy minimisation.

The modern methods which will be discussed here, implement an embedding continuum region modelled explicitly by the finite element method. The advantage of the finite element method for this application is the freedom that the user has in positioning the element nodes at any point in space, 
allowing coincidence of nodes and atoms at the interface if necessary.

The three multiscale models to be discussed in this chapter are the mixed finite element-atomistic (FEAt) method [32], the concurrent coupling of length scales (CLS) method [33, 34] and the quasicontinuum (QC) method [35, 36]. Each of these methods has a region modelled on the atomistic scale by molecular dynamics, and a continuum region modelled by finite element methods. The CLS method also has a tight binding region, however, this will not be discussed here. The way in which these models differ is their respective treatment of interactions at the interface. Molecular dynamics modelling is a non-local atomistic description of particles in a material, whereas finite elements is a local continuum description of a material. When moving from a region with non-local interactions to a region with local interactions, some approximation is needed to overcome the incompatibility. Each of the models introduces a region in which this transition occurs, as shown in figure 4.1.

For all of the models discussed here, there is a direct correspondence between the black atoms and nodes at the interface. Beyond this interface, the atoms within the transition region exist so as to make up for the nonlocal/local mismatch. Depending on the method, some or all of these atoms may be required to coincide with the nodes of the finite elements. Moving away from the interface, elements become increasingly large and accordingly, the node density reduces.

In the following description of the multiscale models, atoms to the left of the interface in figure 4.1 which are in the atomistic region will be called free atoms and labelled with the subscript $F$. The black atoms on the interface which coincide with FE nodes will be called node-atoms and labelled with the subscript $N$. The atoms in the transition region which make up for the non-local/local mismatch will be called imaginary atoms and labelled with 


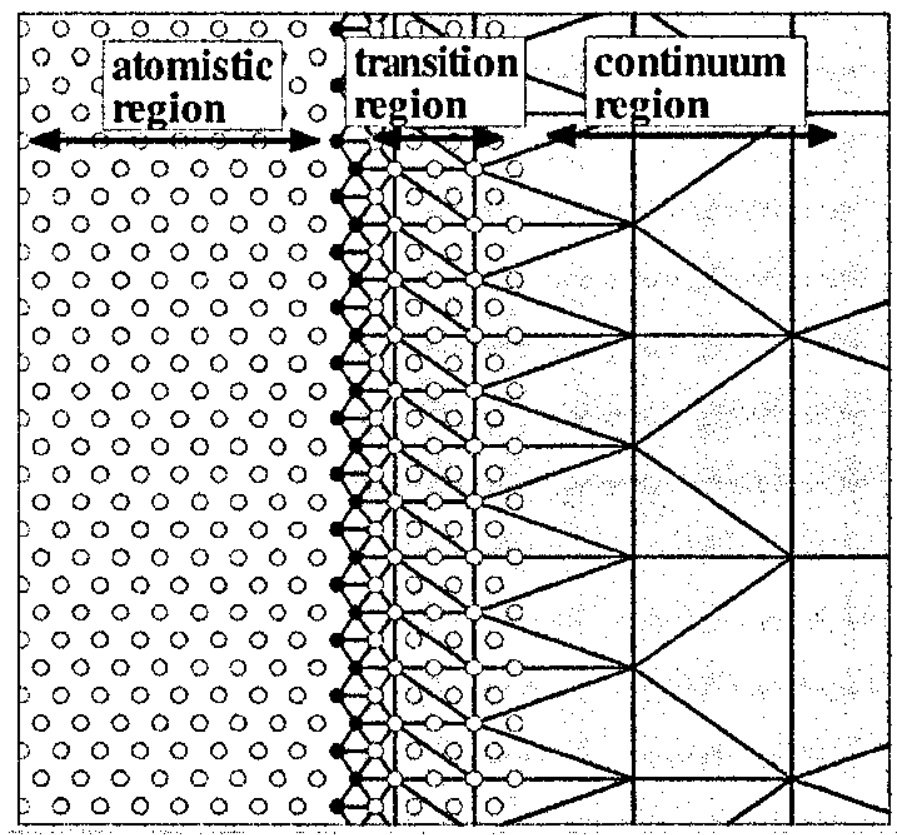

Figure 4.1: A 2D example of a typical coupling setup [28]. The atomistic region on the left is modelled by non-local $\mathrm{MD}$. The continuum region on the right is modelled by local $\mathrm{FE}$. The transition region in the middle uses some approximation to overcome the incompatibility of the two methods. Depending on the method, some or all of the atoms in the transition region may coincide with element nodes.

the subscript $I$.

\subsection{The Mixed Finite Element-Atomistic Method}

The mixed finite element- atomistic method [32] addresses the non-local/local mismatch by introducing a non-local treatment of the continuum in the transition region. A close-up of the transition region and the surrounding area is shown in figure 4.2. Imaginary atoms in the transition region correspond 
directly with the positions of finite element nodes which come down to atomistic spacing at the boundary. Because the imaginary atoms make up the neighbours of free atoms and node-atoms, the size of the transition region is dependent on the potential function used in the atomistic region.

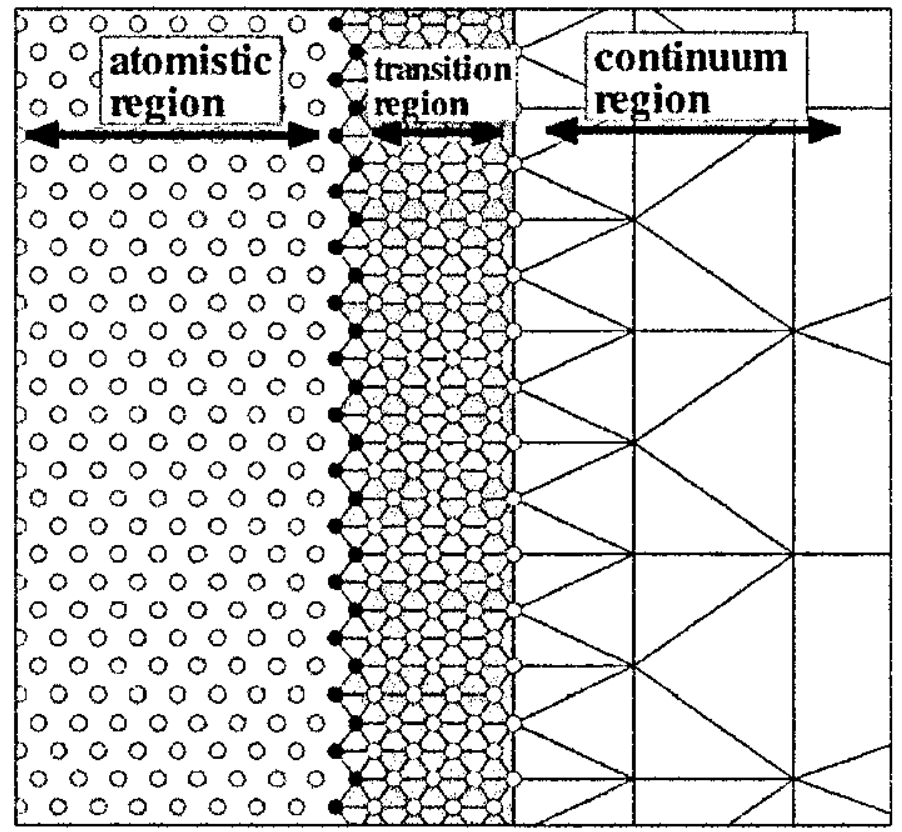

Figure 4.2: A 2D schematic of the FEAt transition region [28]. The white triangular elements in the continuum region use a nonlinear elastic law. The grey elements in the transition region are treated with a non-local modification.

There is no combined total energy function for the FEAt method. Instead, each region is considered separately and provides the boundary condition for the other. The energy sum for the atomistic region in the FEAt method is no different to the total energy calculation for molecular dynamics only simulations. The energy sum, $E^{a}$ includes the position vectors for all free 
atoms, $\mathbf{r}_{F}$, node-atoms, $\mathbf{r}_{N}$, and imaginary atoms, $\mathbf{r}_{I}$,

$$
E^{a}=\sum_{i \in F, N, I} E_{i}\left(\mathbf{r}_{F}, \mathbf{r}_{N}, \mathbf{r}_{I}\right)
$$

In the continuum region, elements which make up the bulk of the region are modelled by non-linear elasticity. Elastic moduli are chosen by first principles or by experiment to match to second order the elastic behaviour of the material in the atomistic region under deformation. Elements which are in the transition region have their energetics described by a non-linear continuum formulation. Instead of the usual local strain energy density, it is defined as an integral over the whole body (although in practice a finite cutoff distance is introduced). The total energy for the continuum region, $E^{c}$, is the sum of the energies of the elements in the transition region described by non-local continuum mechanics, and the sum of the energies of all the other elements not in the transition region described by nonlinear elasticity theory.

$$
E^{c}=\sum_{\mu \in I} E_{\mu}^{(\text {nonloc })}\left(\mathbf{r}_{N}, \mathbf{r}_{I}\right)+\sum_{\mu \notin I} E_{\mu}^{(\text {nonlin })}
$$

where $E_{\mu}^{\text {(nonloc) }}$ is the non-local energy for element $\mu$ in the transition region and $E_{\mu}^{\text {(nonlin) }}$ is the local, nonlinear energy for element $\mu$, not in the transition region.

During a FEAt simulation, the order of computation which leads to the interaction between the models is as follows:

- The node-atoms of the atomistic region provide the fixed nodal positions of the continuum boundary.

- With these fixed boundary positions, a static solution is obtained for the elements in the continuum and transition regions.

- The resulting nodal positions in the transition region give fixed boundary atoms for the next MD time step. 
The correct choice of elastic moduli in the FEAt method results in agreement of elastic properties of the material under investigation in the atomistic and continuum regions. Furthermore, the second order term in the finite element nonlinear elasticity model gives accurate nodal positions in the continuum region for greater distortions than if a linear elasticity model were implemented. However, the static solution obtained at each continuum solution is inconsistent with the dynamic solution of the molecular dynamics model. The boundary atoms for the atomistic region will be fixed at each time step are resolved to their static position, where in reality they would be in motion. Moreover, the coupling method in the FEAt model does not obey Newton's third law of motion of equal and opposite forces. Free atoms just outside of the transition region interact with imaginary atoms within the transition region, however the equal and opposite of this interaction is not applied to the imaginary atom. The positions of nodes in the transition region will differ from those that would be obtained by molecular dynamics and energy will not be conserved.

\subsection{The Concurrent Coupling of Length Scales Method}

The concurrent coupling of length scales (CLS) method [33,34] couples a continuum region to an atomistic region and is modelled as a unified system with a combined energy function. A schematic of the transition region for the CLS method is shown in figure 4.3. The continuum region is described by a linear elastic finite element model with a dynamic solution to match the atomistic solution. The elastic constants for the continuum region are chosen to match those of the atomistic region to minimise the mismatch in 
the models. In the CLS method, the nodal spacing in the transition region must reduce to the atomistic spacing. The atomistic region is modelled by molecular dynamics applied to Si via the Stillinger-Weber potential [37] with two-body and three-body interactions.

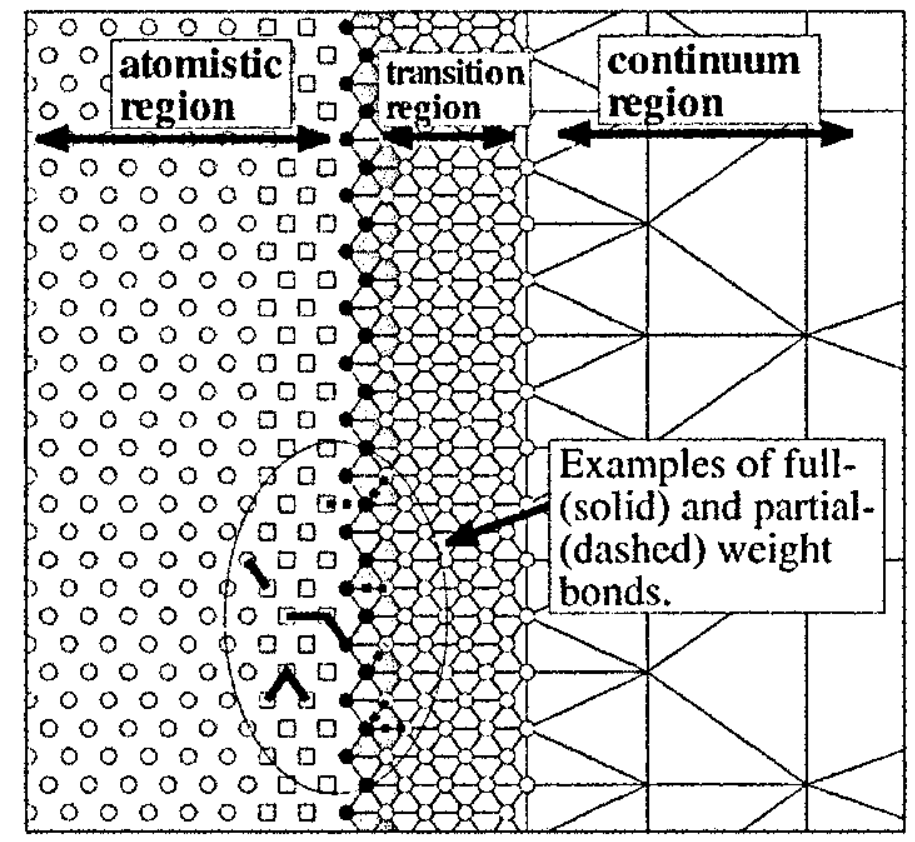

Figure 4.3: A 2D schematic of the CLS transition region [28]. Dashed atomistic bonds contribute half of their energy to the systems total energy, as do the grey elements in contact with the continuum boundary.

In an effort to minimise the non-local/local mismatch, the CLS method used a weighting scheme for interactions that cross the atomistic/continuum boundary. For a two-body interaction between atoms $i$ and $j$, the types of those atoms determine what contribution will be given to the total energy of the system. If both atoms are free atoms, or both atoms are node-atoms, or if one atom is a free atom and one is a node-atom, then the full potential energy, $V_{i j}$, will be counted in the system energy, indicated by the solid lines 
between pairs of atoms in figure 4.3. If one of the atoms is an imaginary atom, but is connected to a free atom or a node-atom, then half of the potential energy will be contributed to the system energy, indicated by dashed lines between pairs of atoms in figure 4.3. If both atoms are imaginary atoms, then no contribution is made to the total system energy.

The three-body interaction contributions are similar to those of the twobody interactions. For a three-body interaction between atoms $i, j$, and $k$, if all three atoms are imaginary atoms then no contribution is made to the system energy. If one or two of the three atoms are imaginary atoms, then half of the potential energy is contributed to the system energy. If all three atoms are either free or node-atoms then all of the potential energy is contributed to the total system energy.

The weighting system is also applied to the energies of the finite elements in the transition region. Most of the elements in this region have nodes which all coincide with imaginary atoms. These elements contribute their whole energy to the total system energy. The elements at the boundary have either one or two nodes which coincide with node-atoms. These elements have only half of their energy given to the total system energy.

The CLS method applies equal and opposite forces to the nodes of the finite elements and to the atoms in the atomistic region and the transition region according to Newton's third law. This ensures that energy is passed correctly through the transition region and energy conservation is achieved for the coupled system. Furthermore, the dynamical solution applied to the continuum region gives a consistent solution to that of the molecular dynamics model. The nodes in the transition region, which provide the boundary condition for the atomistic region, will be in motion during the simulation, as they would if the whole system were modelled by molecular 
dynamics.

The CLS methods coupling technique does introduce some unnatural phenomena. The weighting scheme that is applied at the boundary of the atomistic region and transition region leads to an imbalance of forces on some of the free atoms. The square atoms in the atomistic region of figure 4.3 only experience half of the force that should be felt from three-body interactions from the right. This results in an imbalance in forces at equilibrium, leading to unnatural vibrations. Furthermore, weighted continuum contributions lead to a mismatch of elastic properties in the first layer of elements. Between two nodes, both coinciding with imaginary atoms, no atomistic contribution is applied, however the continuum contribution is still halved.

Another disadvantage of the CLS method is the limitation of the potential functions that can be applied in the atomistic region. The weighting scheme applied to the first layer of elements in the transition region means that atomistic interactions should only be considered up to imaginary atoms within this first layer. Because the nodal spacing must come down to the atomistic spacing, potentials which depend on second neighbour interactions such as the Ackland potential [11] can not be implemented due to the long range embedding function.

\subsection{The Quasicontinuum Method}

The quasicontinuum (QC) method $[35,36]$ was originally developed for two dimensional static equilibrium problems. The method has subsequently been extended to model three dimensional dynamical problems such as nanoindentation $[38,39,40]$.

The multiscale approach defines two types of atom, local representative 
atoms and non-local representative atoms. The non-local description of the material is used in what can be thought of as an atomistic region which is modelled by molecular dynamics. The local description is modelled as a continuum region by finite elements. Figure 4.4 shows the transition region linking the two regions.

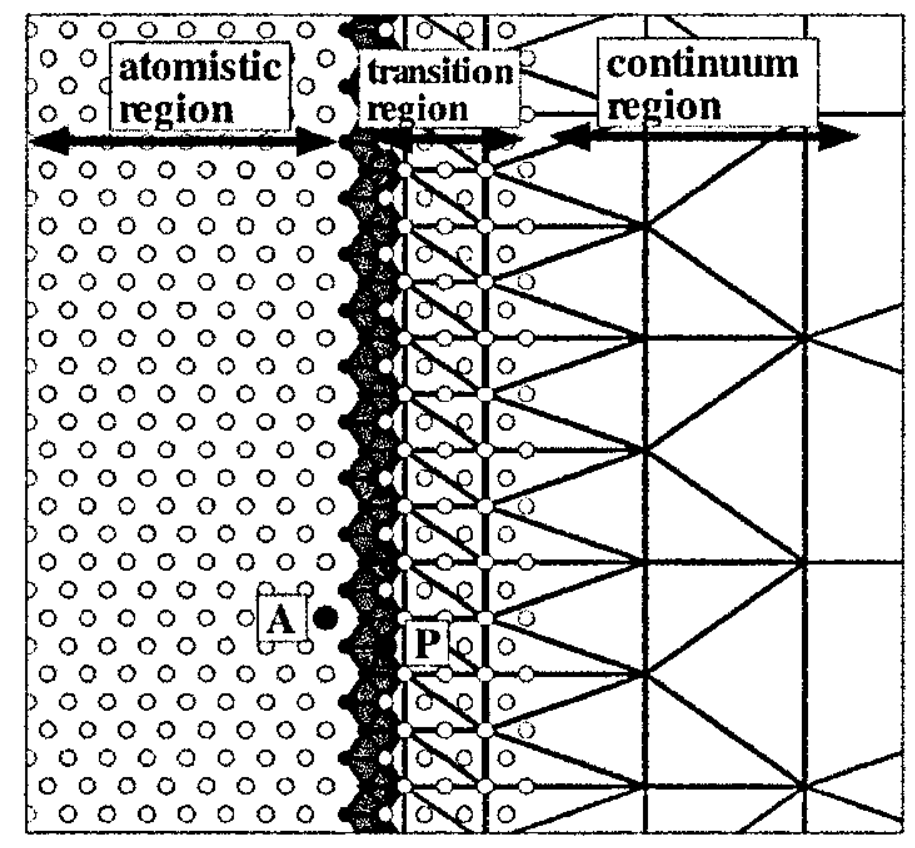

Figure 4.4: A 2D schematic of the QC transition region [28]. Particles A and P experience 'ghost forces' which the QC method balances with a correction term. The grey elements at the interface contribute only part of their energy to the total system energy.

The atomic energies of the free atoms and node-atoms are included in the system energy sum. However, the atomic energies of imaginary atoms are not included in the system energy as these are included in the continuum energy calculation. Hence, interatomic forces due to the energy of an imaginary atom are ignored, but interatomic forces between imaginary atoms and other 
types of atom are included if they come from the energy of a free atom or a node-atom.

To position the imaginary atoms that lie within elements of the transition region, the QC method employs the Cauchy-Born procedure $[41,42,43]$. For each element containing imaginary atoms, the nodal displacements are used to obtain a deformation gradient which is then applied to the internal imaginary atoms across the whole element. The new positions of the imaginary atoms in the transition region provide the boundary condition for the molecular dynamics model of the atomistic region at each time step. The Cauchy-Born procedure is also used by the QC method to calculate the energy $E_{\mu}$ of each element $\mu$ [28].

The QC method attempts to correct for the over counting of energies at the interface by applying a weighting scheme to some of the element energies in the transition region. A weighting factor is defined, $w_{\mu}$, for each element $\mu$, the value of which depends on the number of nodes of the element which are coincident with node-atoms. In the $2 \mathrm{D}$ case with triangular elements, an element with no boundary nodes has weighting factor $w_{\mu}=1$. One boundary node in an element gives a weighting factor of $w_{\mu}=\frac{2}{3}$ and an element with two boundary nodes has a weighting factor $w_{\mu}=\frac{1}{3}$. Similarly for $3 \mathrm{D}$ tetrahedral elements, the weighting factors are $w_{\mu}=1$ when there are no boundary nodes, decreasing by a quarter for each additional boundary node to a maximum of 3 , where the weighting factor is $w_{\mu}=\frac{1}{4}$.

Defining the atomistic energy of an atom $i$ as a function of the atomic positions of all free atoms, $\mathbf{r}_{F}$, node-atoms, $\mathbf{r}_{N}$, and imaginary atoms, $\mathbf{r}_{I}$, the total system energy for the QC method is given as

$$
E_{Q C}=\sum_{i \in(F, N)} E_{i}\left(\mathbf{r}_{F}, \mathbf{r}_{N}, \mathbf{r}_{I}\right)+\sum_{\mu} w_{\mu} E_{\mu}
$$

The approximation made by the QC method in moving from a non-local 
description to a local description of energies and forces is to ignore the atomic energies of imaginary atoms. The result of this approximation is the imbalance of forces, and hence unphysical motion of particles at equilibrium positions. The unphysical forces experienced in equilibrium positions are called 'ghost forces'. Atoms near the interface (such as atom A in figure 4.4) are not treated correctly as real atoms. This is because the forces on these atoms due to the motion of imaginary atoms (such as P in figure 4.4) are ignored, and hence ghost forces are generated. Similarly, forces due to atoms near the interface, such as atom A, must exert an equal and opposite force on imaginary atoms such as atom $\mathrm{P}$ in order to obey Newton's third law of motion and hence to give energy conservation. This results in unphysical nodal ghost forces in the transition region when the nodes are at their equilibrium positions.

The QC method correction of ghost forces [44] deals with the unphysical force imbalance by the following procedure:

- All atoms and nodes are placed at their initial equilibrium positions (where forces should be zero).

- The ghost forces are resolved for all free atoms and nodes of elements in the transition region.

- The negatives of the ghost forces are stored to be applied to their respective atom/node as correction forces at each step of the simulation.

- The energy function for the QC method is adjusted for the work done by the correction forces, given as

$$
E_{Q C}^{\prime}=E_{Q C}-\sum_{k} g_{k}^{0} u_{k}
$$


where $g_{k}^{0}$ is the ghost force on atom/node $k$, and $u_{k}$ is the node/atom's displacement.

One of the main advantages of the QC method is its transferability. The interpolation of imaginary atom positions to make up neighbours of free atoms allows any potential function with a finite cut-off distance to be implemented. The method applies equal and opposite forces to all particles according to Newton's third law and hence energy conservation is achieved. Furthermore, the correction of ghost forces in the QC method removes the unphysical imbalance of forces at equilibrium positions. These corrective forces are applied to nodes of the finite elements and to atoms in the atomistic region. If linear elasticity theory is applied to the continuum region then the constant force applied to the nodes is correct as the force is proportional to the displacement. The atoms, on the other hand, have a nonlinear description of force with respect to displacement and the correction force is therefore only correct at equilibrium. Another disadvantage of the QC method is the weighting applied to element energies at the interface. Interactions between pairs of imaginary atoms are ignored in the QC method, so when the weighting factor is applied to the element, there is a resulting mismatch in the material properties between the atomistic region and the first layer of elements in the transition region.

\subsection{Conclusions}

Although all three of the methods described here provides a coupling scheme to bridge the length scales in materials modelling, each has its disadvantages. Both the FEAt method and the CLS method require that the nodal spacing reduces to the atomistic spacing in the transition region. For simple crystal 
structures this is not a problem, however, when modelling a more complex structure, such as an amorphous material or a spinel [45], the tessellation of the cell becomes considerably more complicated. The FEAt method has a static solution at each step of the simulation in the continuum region, in disagreement with the dynamical MD solution in the atomistic region. Newton's third law of equal and opposite forces is not obeyed at the interface between the models, and hence the FEAt method can not achieve energy conservation when the system is disturbed. The CLS method and the QC method both have coupling techniques which produce ghost forces in the transition from non-local to local descriptions of the material. In the CLS method, these forces are not corrected and result in unnatural vibrations. The QC method's ghost force correction scheme removes these forces at equilibrium, but the linear correction is inappropriate to apply to the nonlinear atomistic region. Furthermore, the weighting schemes that both the CLS and QC methods apply to the first layer of elements in the transition region generate a mismatch in elastic constants between these elements and the atomistic region.

In the following two chapters of this work a multiscale model is developed, first in $2 \mathrm{D}$ and subsequently in $3 \mathrm{D}$, which addresses the disadvantages of previous methods described above. The objectives in developing this new model are to create a coupling technique which is simple to implement and gives a physically accurate description of the material across the interface of the method. 


\section{Chapter 5}

\section{Two Dimensional Multiscale Modelling}

The coupling of atomistics to continuum mechanics in 2D is studied in this chapter. Two multiscale coupling techniques are described and tested, both of which link a molecular dynamics model to a 2D linear elastic finite element model. As well as the coupling techniques themselves, a 2D meshing algorithm is described, specifically for the purpose of embedding atomistics within a finite element continuum.

In materials modelling simulations of experiments such as nanoindentation or laser ablation, 2D models cannot properly describe the material behaviour and 3D models are generally required to describe correctly the resulting physical phenomena. Although 2D models are insufficient to describe these 3D experiments, they can be useful during the development of a new technique as the methodology is generally simpler to implement than in the 3D models. Furthermore fewer particles need to be modelled, both of which ease the debugging process. 


\subsection{Meshing Technique for Embedding Atom- istics in $2 \mathrm{D}$}

Generating a suitable mesh over any irregular shape is never a trivial exercise. Multiscale models which couple an atomistic region to a continuum region may impose an additional constraint that the finite element nodes are required to coincide with some or all of the atomistic positions in the transition region. This is largely dependent on the coupling method that is used.

When performing simulations such as nanoindentation, the strain field produced in the atomistic region which propagates into the continuum region becomes smaller the further the node is from the atomistic region. This makes it possible to increase the size of elements in the far field and therefore reduce the density of nodes with distance from the interface. Reducing the number of nodes in this manner reduces the computational expense at each time step.

Generating a mesh which surrounds a square atomistic region could be achieved in a number of ways. The method used here is to generate a mesh over an eighth of the continuum region (shown in figure 5.1 (a)). This section is then reflected and transformed to cover the entire region, as shown in figure 5.1 (b).

The section of the continuum region in figure 5.1 (a) is meshed based on a modification to the simple automatic meshing algorithm described by Cheung [46]. Nodes are positioned on the trapezium such that there are $N_{x}$ nodes on the short inside edge and $N_{e}$ on the long outside edge. The number of layers from the inside edge to the outside edge is set to be $N_{x}$, so the number of nodes on any given layer, $N Y_{j}$ is given by

$$
N Y_{j}=N_{x}-\left|\frac{\left(N_{x}-N_{e}\right)(j-1)}{N_{x}-1}\right|
$$




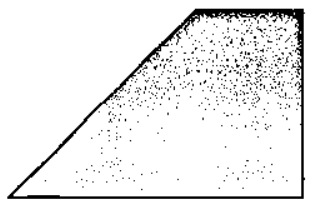

(a)

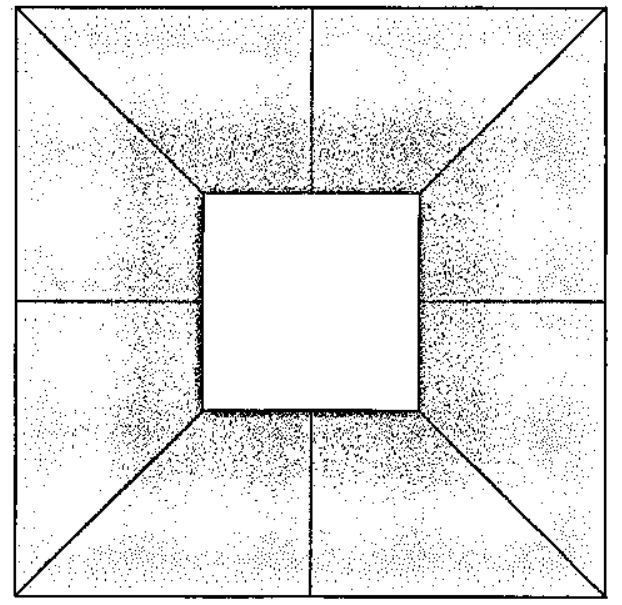

(b)

Figure 5.1: Mesh Generation and Node Density. (a) The node placement and connectivity is completed for an eighth of the continuum region, (b) it is then repeated to embed the square atomistic region.

The entire continuum region (figure 5.1 (b)) is initially created with sides of length 1 , and the inner hole for the atomistic region with user defined length $d$. The region is subsequently scaled up to match atom positions on the inside edges. The $y$ values for the $N_{x}$ layers of nodes are calculated by

$$
\begin{aligned}
& Y_{1}=\frac{1-d}{2} \\
& Y_{j}=Y_{j-1}-\frac{Y_{1} s_{j}}{s_{T}} \quad\left(j=2 \ldots N_{x}\right)
\end{aligned}
$$

where the $s_{j}$ values vary the rate of change in spacing between layers and are defined by the geometric progression

$$
\begin{aligned}
s_{1} & =0, s_{2}=1 \\
s_{j} & =C s_{j-1} \\
s_{T} & =\sum_{j=1}^{N_{x}} s_{j}
\end{aligned} \quad\left(j=3 \ldots N_{x}\right)
$$


where $C$ is a magnification factor. If $C=1$, the spacing between layers remains constant. Choosing a value larger than 1 gives a spacing that becomes progressively longer, and hence reduces the node density towards the outside edge.

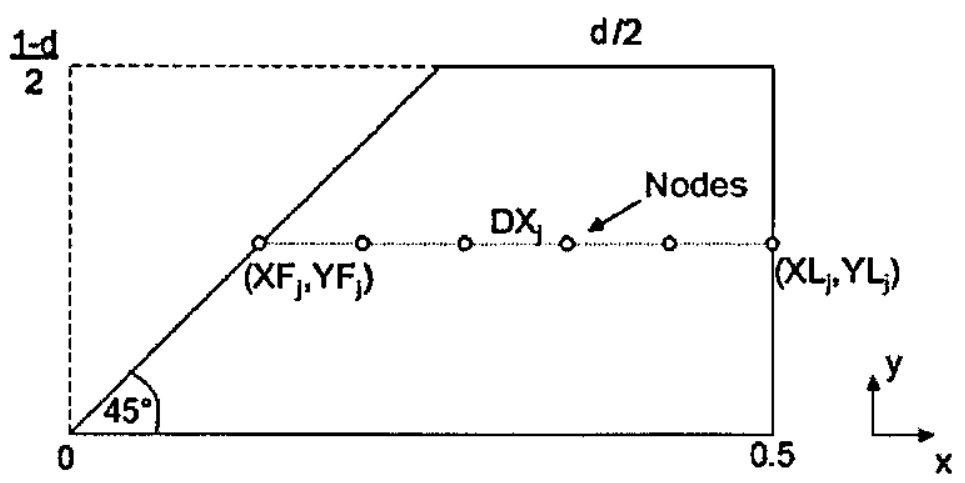

Figure 5.2: Node positioning in the trapezium. The number of nodes is reduced at each layer from the inside edge to the outside edge and are spaced equally along each layer.

Figure 5.2 shows the node spacing for a particular layer. The $y$ coordinates of the start and end of the line, $Y F_{j}$ and $Y L_{j}$, are both given by $Y_{j}$, which is also the same as the $x$ coordinate at the start of the line, $X F_{j}$, due to the $45^{\circ}$ angle. The $x$ value at the end of the line $X L_{j}=0.5$ due to the vertical edge half way along the unit square of the continuum region. Equal spacing between nodes along the line are calculated by

$$
D X_{j}=\frac{X L_{j}-X F_{j}}{N Y_{j}-1}
$$

then the nodal position $\left(x_{i j}, y_{i j}\right)$ of a node $(i, j)$ is given by

$$
\begin{aligned}
& y_{i j}=Y_{j} \\
& x_{i j}=X F_{j}+(i-1) D X_{j} \quad\left(j=1 \ldots N_{X}, i=1 \ldots N Y_{j}\right)
\end{aligned}
$$


Nodes in neighbouring layers are used to make up triangular elements to tessellate the space between layers. When the number of nodes in the two layers are equal $\left(N Y_{j+1}=N Y_{j}\right)$, the meshing is applied as shown in figure 5.3 (a), where opposite corners $\mathrm{A}$ and $\mathrm{C}$ are connected to give more regular shaped elements as the distance AC is shorter than distance BD. However, if the number of nodes is reduced between layers $\left(N Y_{j+1}=N Y_{j}-1\right)$, the final 3 nodes in layer $j$ are connected to the final 2 nodes in layer $j+1$ according to figure $5.3(\mathrm{~b})$.

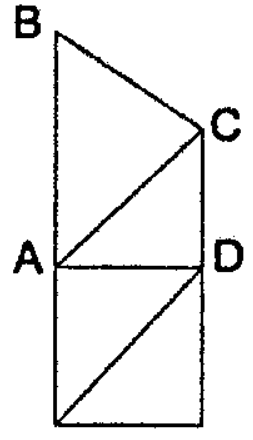

(a)

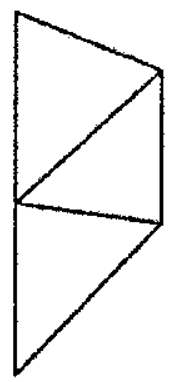

(b)

Figure 5.3: 2D tessellation between layers of nodes. (a) The number of nodes in neighbouring layers is equal. (b) The number of nodes is reduced between layers.

The variable shading in figure 5.1 indicates the node density. The density of nodes increases as the shading becomes darker around the square atomistic region in the middle. A typical mesh will have 100 nodes on each inside edge to coincide with a $100 \times 100$ square of atoms, with elements expanding to the outside boundary where each side is 10 times longer than on the inside and has just 10 nodes. The increase in length and reduction of nodes on the 
outside edges leads to the spacing between nodes increasing 100 fold.

\subsection{Matching Elastic Constants in 2D}

In a multiscale model which couples finite elements to molecular dynamics, the description of the elastic behaviour of the material under investigation is governed by the different parameters in the finite element model and the potential function implemented in the molecular dynamics model. In order to model correctly the material across the interface, it is important to match the elastic properties in the finite element model with the molecular dynamics model to prevent reflection and diffraction when strain fields or elastic waves pass from one region to another. This is possible by calculating the Young's modulus and Poisson's ratio in terms of the parameters of the potential function used in the molecular dynamics model. Figure 5.4 shows a $n \times m$ grid of atoms in a simple square lattice which, for the $2 \mathrm{D}$ development work in this chapter will have interatomic forces calculated via the Lennard-Jones potential described in section 2.2.1 and will be distorted to determine the elastic properties.

The Young's modulus of a material may be calculated by dividing the stress applied to the material by the strain induced by that stress.

$$
E=\frac{\text { stress }}{\text { strain }}=\frac{\frac{F}{A}}{\frac{\Delta l}{l_{0}}}
$$

The cross-sectional area of the 'beam' in figure 5.4 is given by $A=m r_{0} t$, the natural length of the 'beam' is $l_{0}=n r_{0}$ where $r_{0}$ is the atom's equilibrium separation, and $\Delta l$ is the beam's elongation due to the total force on the beam, $F=m F_{a}$, shown in figure 5.5 , where $F_{a}$ is the applied external force. 


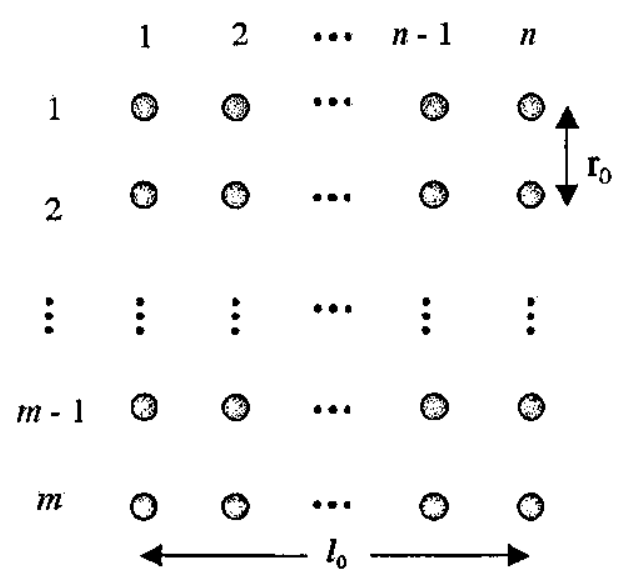

Figure 5.4: $n \times m$ grid of atoms

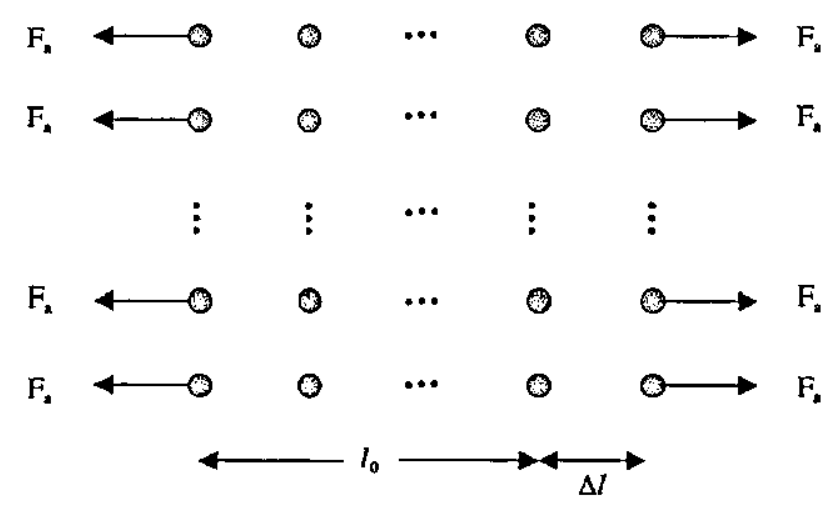

Figure 5.5: Elongation of an $n \times m$ grid of atoms due to an applied force at the ends.

The force on each of the end atoms is the only remaining unknown and should be calculated in terms of whichever potential function has been used. By taking the Taylor series expansion of the potential function $\mathcal{V}(r)$,

$$
\mathcal{V}\left(r_{0}+\Delta r\right)=\mathcal{V}\left(r_{0}\right)+\Delta r \mathcal{V}^{\prime}\left(r_{0}\right)+\frac{\Delta r^{2}}{2} \mathcal{V}^{\prime \prime}\left(r_{0}\right)+\ldots
$$

ignoring higher order terms and noting $\mathcal{V}^{\prime}\left(r_{0}\right)=0$, the force between two atoms separated by $\Delta r(=\Delta l / n)$ is 


$$
F_{a}=\frac{d \mathcal{V}}{d(\Delta r)}=\Delta r \mathcal{V}^{\prime \prime}\left(r_{0}\right)
$$

Making these substitutions into equation 5.7 leaves an expression for the Young's modulus in terms of the potential function and the thickness of the material, $t$. The value chosen for this thickness is not important as it is eliminated when constructing the stiffness matrix in the finite element model.

$$
E=\frac{m \mathcal{V}^{\prime \prime}\left(r_{0}\right) \frac{\Delta l}{n} n r_{0}}{m r_{0} t \Delta l}=\frac{\mathcal{V}^{\prime \prime}\left(r_{0}\right)}{t}
$$

For the Lennard-Jones 6-12 potential, the equilibrium separation is $r_{0}=$ $\sqrt[6]{2} \sigma$ and the second derivative of the potential function is given by

$$
\mathcal{V}_{L J}^{\prime \prime}(r)=4 \epsilon\left(\frac{156 \sigma^{12}}{r^{14}}-\frac{42 \sigma^{6}}{r^{8}}\right) .
$$

Substituting $r_{0}$ into the second derivative of the potential function, then the solution into equation 5.10, the Young's modulus in terms of the LennardJones potential parameters is

$$
E=\frac{36 \sqrt[3]{4}}{t} \frac{\epsilon}{\sigma^{2}} .
$$

When considering the Lennard-Jones 6-12 potential on a $2 \mathrm{D}$ simple square lattice, the uniform elongation of the atoms in one direction will result in no change in the other giving a Poisson's ratio $\nu=0$.

\subsection{Finite Element Time Integration}

The finite element model for linear elasticity described in section 2.3 is conventionally solved to find displacements caused by some applied force. This static solution represents the total relaxation of the system and is inconsistent with the dynamic solution of the molecular dynamics model. 
In order to obtain a dynamic solution in the continuum region a modification to the FE model is required. By considering that any node may already be displaced at a particular time step of a simulation, a resulting restoration force on the node is required to apply the velocity Verlet algorithm (see section 2.1) and move the FE system forward in time. In standard FE packages, equation 2.48 is usually solved for displacements Q. However, it is possible to calculate the resultant force on each node due to the fact that it is displaced from its equilibrium position by equation 5.13 .

$$
\mathbf{F}=-\mathbf{K Q}
$$

Applying the same numerical integrator to advance the continuum region as is used in the atomistic region makes the dynamical solution in the two regions consistent with each other. The acceleration of the node is calculated in the usual way, $a=\frac{F}{M_{n d e}}$. For $2 \mathrm{D}$ triangular elements, the mass of the node, $M_{n d e}$, is based upon a third of the area of its surrounding elements, splitting the mass of the element between each of its three nodes. Say a particular node is surrounded by $n$ elements, $e_{1} \ldots e_{n}$, with areas $A_{e_{1}} \ldots A_{e_{n}}$, its mass is

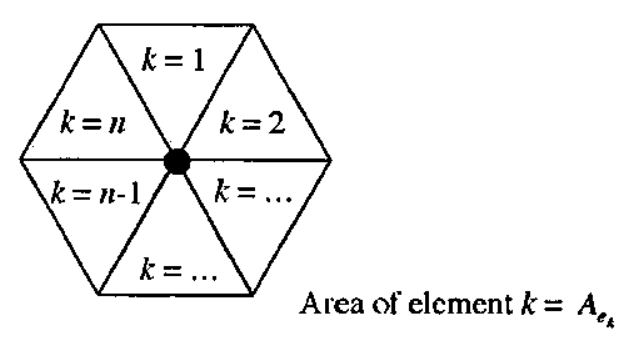

Figure 5.6: Calculating the mass of a node. For 2D triangular elements, a third of the mass of the elements is attributed to each of the three nodes.

calculated by equation 5.14 , where $\rho$ is the mass per unit area. This density can be calculated by considering the mass of an atom and how many atoms 
there are in a unit square.

$$
M_{n d e}=\rho \sum_{k=1}^{n} \frac{A_{e_{k}}}{3}
$$

\subsection{Initial Coupling Method for up to Second Nearest Neighbours}

In this section, a method is described for coupling first and second nearest neighbour atomistics to a continuum model in the far field. Newton's third law of motion states that for every action, there is an equal and opposite reaction. Independently, both the finite element method and molecular dynamics model obey this law, however, when coupling the two models to create a multiscale model this is not guaranteed and some existing methods fail in this regard. Care must be taken to ensure that forces at the interface are dealt with in agreement with Newton's third law. This is a main consideration in developing the method that follows.

\subsubsection{Methodology}

When calculating the forces on each finite element node or molecular dynamics atom in the coupled system, if the force on atom/node $i$, due to atom/node $j$ is defined as $F_{i j}$, then for pair potentials, Newton's third law is obeyed iff $F_{i j}=-F_{j i}$. One way to make certain of this is to ensure that the same model (FE or MD) is used to calculate the force between each pair of particles. A technique to do this is described in this section for the cases where a pairwise potential function cut-off includes first nearest neighbours in the normal crystal structure. A method where second neighbours are 
included is also developed.

Figure 5.7 shows the positions of nodes and atoms at the coupled interface. Finite elements are represented as white triangles with crosses indicating the FE nodes, and atoms are represented by grey circles in this model. At the interface, there is a direct positional correspondence between atoms and nodes, hence the nodal spacing must reduce to the atomic spacing. This may be achieved by applying the correct scaling of the continuum region described in section 5.1. The FE interactions in the continuum region are entirely included in the coupled model where the connectivity of the system defines which nodes interact with each other. A modification is made to the $\mathrm{MD}$ model interaction along the interface to account for the FE interactions. If a pair of atoms that would normally interact via $\mathrm{MD}$, both coincide with a pair of connected nodes, the MD interaction is omitted from the model.

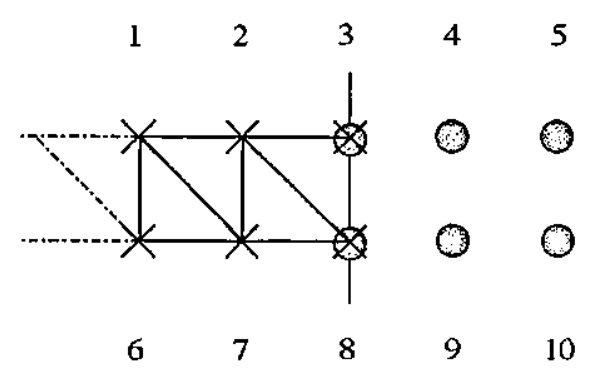

Figure 5.7: Schematic of the coupled interface in 2D. FE nodes (X) coincide with MD atoms at the interface.

Table 5.1 indicates which model is used to calculate the particle forces present for first nearest neighbour atomistic and continuum interactions of the particles in figure 5.7. The method used to calculate the force on atom/node $i$ due to atom/node $j$ is placed in table cell $i j$, described as ' $\mathrm{FE}$ ' if the force is calculated by the finite element method, or 'MD' if the force is calculated by the molecular dynamics model. The symmetry of table 5.1 verifies that 
Newton's third law has been obeyed in the first nearest neighbour case.

\begin{tabular}{|c||c|c|c|c|c|c|c|c|c|c|}
\hline$i \backslash j$ & 1 & 2 & 3 & 4 & 5 & 6 & 7 & 8 & 9 & 10 \\
\hline \hline 1 & - & $\mathrm{FE}$ & - & - & - & $\mathrm{FE}$ & $\mathrm{FE}$ & - & - & - \\
2 & $\mathrm{FE}$ & - & $\mathrm{FE}$ & - & - & - & $\mathrm{FE}$ & $\mathrm{FE}$ & - & - \\
3 & - & $\mathrm{FE}$ & - & $\mathrm{MD}$ & - & - & - & $\mathrm{FE}$ & - & - \\
4 & - & - & $\mathrm{MD}$ & - & $\mathrm{MD}$ & - & - & - & $\mathrm{MD}$ & - \\
5 & - & - & - & $\mathrm{MD}$ & - & - & - & - & - & $\mathrm{MD}$ \\
6 & $\mathrm{FE}$ & - & - & - & - & - & $\mathrm{FE}$ & - & - & - \\
7 & $\mathrm{FE}$ & $\mathrm{FE}$ & - & - & - & $\mathrm{FE}$ & - & $\mathrm{FE}$ & - & - \\
8 & - & $\mathrm{FE}$ & $\mathrm{FE}$ & - & - & - & $\mathrm{FE}$ & - & $\mathrm{MD}$ & - \\
9 & - & - & - & $\mathrm{MD}$ & - & - & - & $\mathrm{MD}$ & - & $\mathrm{MD}$ \\
10 & - & - & - & - & $\mathrm{MD}$ & - & - & - & $\mathrm{MD}$ & - \\
\hline
\end{tabular}

Table 5.1: First nearest neighbour atomistic and continuum interactions for particles in figure 5.7. The symmetry indicates that Newton's third law is obeyed.

Table 5.2 indicates the model used to calculate particle forces present for second nearest neighbour atomistic interactions and first nearest neighbour continuum interactions of the particles in figure 5.7. Consider, for example, interface point 8 , the first nearest neighbours are points 3,7 and 9 , and the second nearest neighbours are points 2 and 4 . The forces between point 8 and points 2,3 and 7 can be calculated using the finite element model because there are elements which connect these points. There are no such connections between point 8 and points 4 and 9 , so the atomistic potential must be used to calculate the forces between these points. Table 5.2 is again symmetric and hence the coupled model obeys Newton's third law. 


\begin{tabular}{|c||c|c|c|c|c|c|c|c|c|c|}
\hline$i \backslash j$ & 1 & 2 & 3 & 4 & 5 & 6 & 7 & 8 & 9 & 10 \\
\hline \hline 1 & - & $\mathrm{FE}$ & - & - & - & $\mathrm{FE}$ & $\mathrm{FE}$ & - & - & - \\
2 & $\mathrm{FE}$ & - & $\mathrm{FE}$ & - & - & - & $\mathrm{FE}$ & $\mathrm{FE}$ & - & - \\
3 & - & $\mathrm{FE}$ & - & $\mathrm{MD}$ & - & - & - & $\mathrm{FE}$ & $\mathrm{MD}$ & - \\
4 & - & - & $\mathrm{MD}$ & - & $\mathrm{MD}$ & - & - & $\mathrm{MD}$ & $\mathrm{MD}$ & $\mathrm{MD}$ \\
5 & - & - & - & $\mathrm{MD}$ & - & - & - & - & $\mathrm{MD}$ & $\mathrm{MD}$ \\
6 & $\mathrm{FE}$ & - & - & - & - & - & $\mathrm{FE}$ & - & - & - \\
7 & $\mathrm{FE}$ & $\mathrm{FE}$ & - & - & - & $\mathrm{FE}$ & - & $\mathrm{FE}$ & - & - \\
8 & - & $\mathrm{FE}$ & $\mathrm{FE}$ & $\mathrm{MD}$ & - & - & $\mathrm{FE}$ & - & $\mathrm{MD}$ & - \\
9 & - & - & $\mathrm{MD}$ & $\mathrm{MD}$ & $\mathrm{MD}$ & - & - & $\mathrm{MD}$ & - & $\mathrm{MD}$ \\
10 & - & - & - & $\mathrm{MD}$ & $\mathrm{MD}$ & - & - & - & $\mathrm{MD}$ & - \\
\hline
\end{tabular}

Table 5.2: Second nearest neighbour atomistic interactions and first nearest neighbour continuum interactions for particles in figure 5.7. The symmetry indicates that Newton's third law is obeyed.

\subsubsection{Testing and Results}

To test the $2 \mathrm{D}$ coupling technique, a suitable procedure is required. One objective of creating a coupled model is to span the length scales when modelling nanoindentation, hence a $2 \mathrm{D}$ version of this experiment is used to test the model here. A single layer of atoms surrounded by a 2-dimensional continuum surface loosely represents a 'thin film'. Slowly forcing an indenter into the atomistic region of the film produces a strain field which moves across the interface of the coupled model into the continuum region. The dynamic visualisation of the simulation allows analysis of the coupling technique as the field crosses the models interface.

For this 2-dimensional case the depth of the indenter into the atomistic 
region is described by the simple quadratic function 5.15 where $\frac{l}{2}$ is the distance from the $x$ and $y$-axes to the paraboloid's central axis, as depicted in figure 5.8 .

$$
I(x, y, t)=\frac{1}{\beta}\left[\left(x-\frac{l}{2}\right)^{2}+\left(y-\frac{l}{2}\right)^{2}-\alpha(t)\right]
$$

The parameter $\alpha(t)$ changes linearly with time from zero at the start of the simulation, to a maximum value of $\alpha_{\max }$ after some specified time into the simulation, causing the tip of the indenter to lower to a depth of $d_{\max }$. By

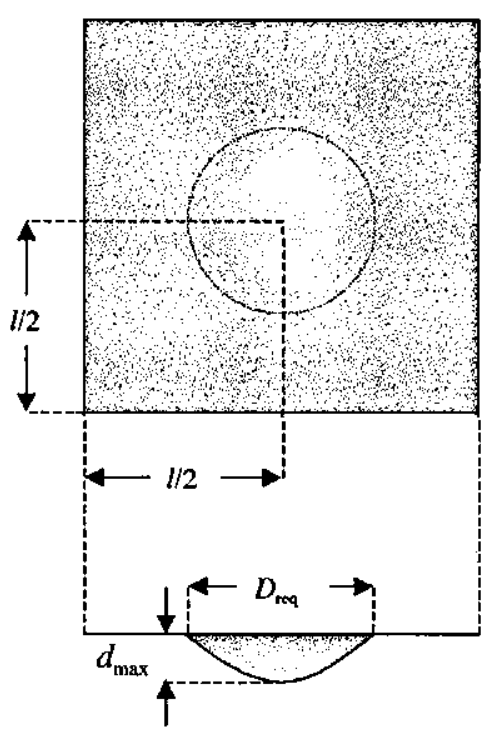

Figure 5.8: Thin film indentation used to test the 2D model.

considering the required indenter depths at the central axis and the required diameter, $D_{\text {req }}$, it is easy to find expressions for $\alpha_{\max }$ and $\beta$.

$$
\begin{gathered}
\alpha_{\max }=\left(\frac{D_{r e q}}{2}\right)^{2} \\
\beta=\frac{\alpha_{\max }}{d_{\max }}
\end{gathered}
$$


At each time step of the simulation $\alpha(t)$ is updated and a new value of $I(x, y, t)$ is calculated for each atom using the atom's original $x$ and $y$ coordinates. If this value is negative, the atom's $z$ coordinate is set to this new value, causing an indentation into the atomistic region. The 2-dimensional finite element model only models displacements propagated in the $x$ and $y$ directions, hence the displacement in the $z$ direction should be small.

The parameters in table 5.3, have been used to produced the results shown in this section, giving 10,000 atoms and 10,952 nodes. To model this size of plane using molecular dynamics alone would require approximately $1,000,000$ atoms.

\begin{tabular}{c|c}
\hline Parameter & Value \\
\hline \hline Atoms & 10,000 \\
Inside edge nodes & 100 \\
Outside edge nodes & 10 \\
Total nodes & 10,952 \\
Elements & 21,488 \\
Time step & $1 \mathrm{fs}$ \\
Indenting time & $50 \mathrm{ps}$ \\
Total time & $400 \mathrm{ps}$ \\
Indent depth, $\frac{\alpha_{\max }}{\beta}$ & $0.25 \AA$ \\
Indent diameter & $25 \AA$ \\
\hline
\end{tabular}

Table 5.3: Parameters used for the 2D thin film indentation test simulation.

Snapshots have been taken at various points during the simulation and are displayed below in figure 5.9. The atomistic region, displayed to the left, comes from the blank square of the corresponding continuum region to the right. After 150ps, the atomistic region shows little change and therefore 
figures 5.10 and 5.11 only display the continuum region. The colour ranges from blue, for no displacement, to red for displacement of $1 \times 10^{-2} \AA$.

The image in figure 5.9(a) taken at 40ps shows the indentation in the centre of the atomistic region. Besides the central depressed area, atoms displacements propagate out along directions parallel to the crystal lattice directions, this is due to the square lattice and the first nearest neighbour interactions in the molecular dynamics model. At 40ps these displacements have just crossed the interface into the continuum region, shown in figure 5.9(a) at the centre of each inside edge. The two figures 5.9(b) and 5.9(c) at 70ps and 110ps respectively, show an increase in the displacement and further propagation across the interface, without reflection or disturbance.

Figure 5.9(d) depicts the simulation 100ps after the indenter stopped moving into the film and the displacement field around the indentation shows no significant change as the simulation progresses. In the continuum region, waves have formed from the displacement field and are moving away from the atomistic region.

Interference patterns due to the reflected wave interactions are visible in both figures 5.10 and 5.11. First, waves cross on their way to the outside boundary as in figure 5.10 at $225 \mathrm{ps}$, the waves then reflect off the outside boundary and more interference occurs, as is the case at the end of the simulation in figure 5.11 .

\subsubsection{Conclusions}

The results from the simple test of the $2 \mathrm{D}$ model show that the coupling technique allows interaction between the atomistic and continuum regions. The strain field created by the indentation in the atomistic region is transmitted to the continuum region across the interface, then the elastic waves 

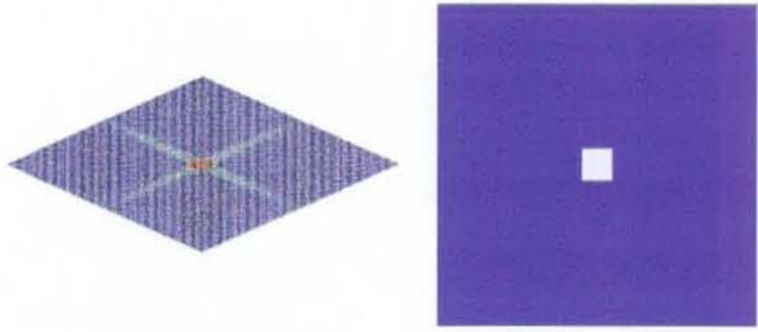

(a) $40 \mathrm{ps}$

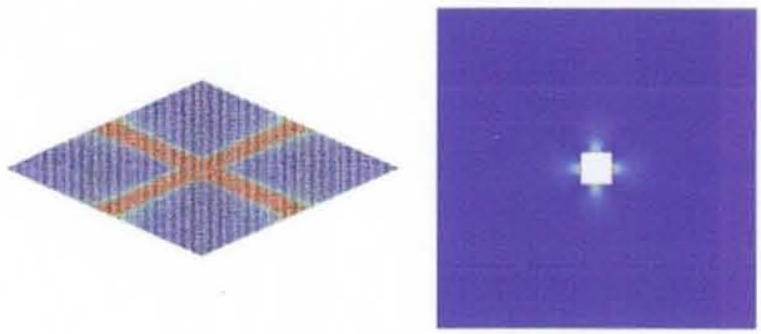

(c) $110 \mathrm{ps}$
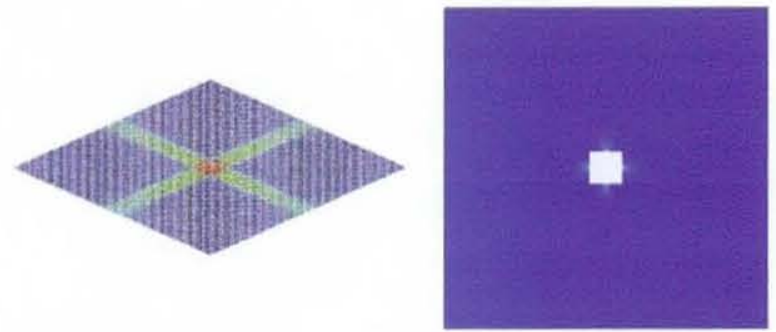

(b) $70 \mathrm{ps}$

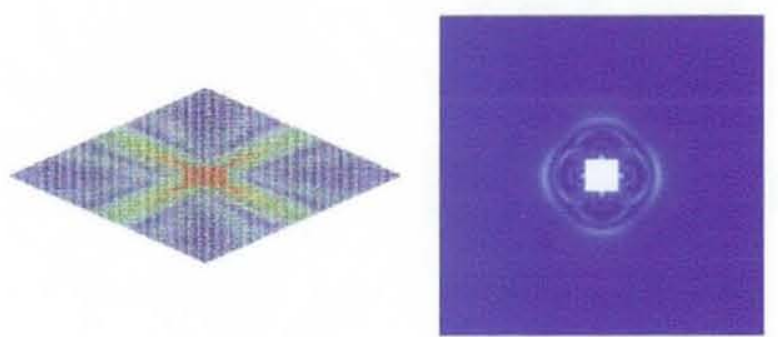

(d) $150 \mathrm{ps}$

Figure 5.9: Images from the test simulation at various time steps. (a) The strain field can clearly be seen in the MD region, but is yet to cross into the continuum region. (b) The strain field has begun to cross the interface into the continuum region. (c) Larger displacements are visible further into the continuum region. (d) The motion of material due to the indentation generates elastic waves in the continuum region. These are fed back into the atomistic region via the coupled interface.

generated in the continuum region are fed back into the atomistic region. However, this method is limited to first and second nearest neighbour interactions of a simple square 2D lattice. Beyond this, when considering third nearest neighbours or different 2D lattice structures such as 2D close packed, the method fails. This is due to the interactions which are required for MD 


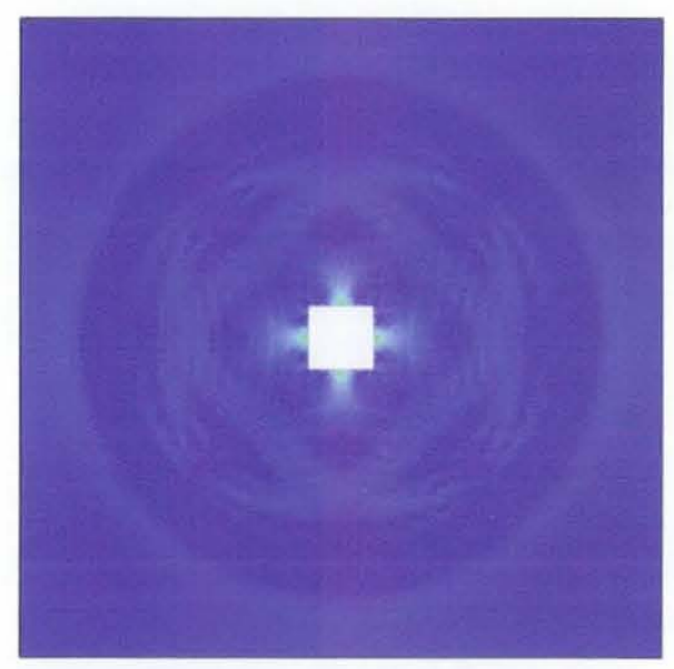

Figure 5.10: Snapshot at 225ps. Interference patterns are visible where elastic waves have crossed one another.

atoms with particles within the continuum, which under this scheme would already be accounted for by the FE model.

In order to progress to multiscale modelling in $3 \mathrm{D}$, which for realistic potential functions will need to include interactions that cross the interface, a more generic model is required that is able to incorporate any crystal structure or potential function.

\subsection{Position Matching Generic Coupling Tech- nique in $2 \mathrm{D}$}

Most interatomic potential functions, some of which are described in section 2.2 , have a finite cut-off distance beyond which the interatomic forces are 


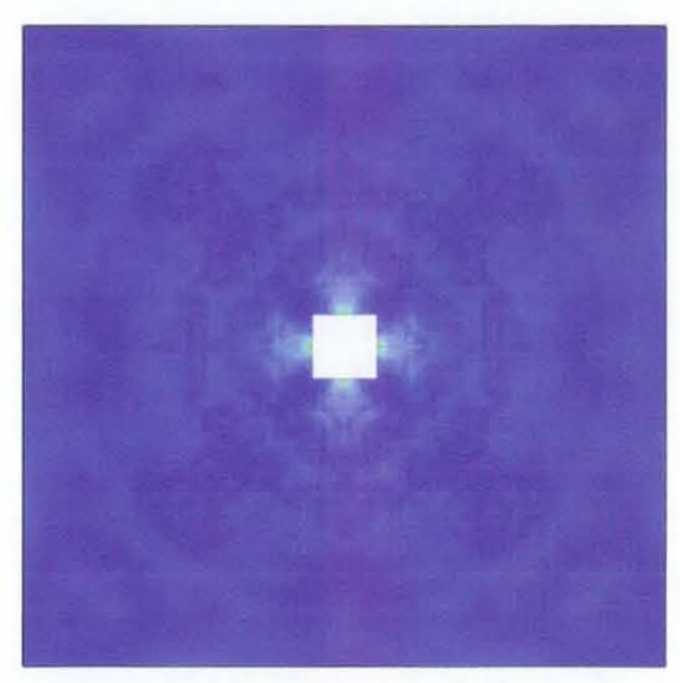

Figure 5.11: Snapshot at 400ps. The waves bounce off the outside boundary of the continuum region creating further interference patterns.

neglected. In this section, a coupling method linking a non-local molecular dynamics description of an atomistic region to a local finite element continuum region is described which has been developed to take advantage of this cut-off distance. The interface of the two regions is not as straightforward as in the previous method, however, this is necessary in order to allow realistic potentials with long range interactions to be applied. The method is generic in that any potential function with a finite cut-off distance may be applied in the atomistic region.

\subsubsection{Methodology}

The main objective of this method is to address the issue of moving from a non-local description of the atomistic region, to a local description of the 
continuum region. The coupling method introduces an overlap of the MD atoms and the continuum elements where this transition will occur. This overlap of the two models will be referred to as the transition region. A schematic representation at the interface of the coupled model is shown in figure 5.12. In this model, there are two main types of atom. Atoms which have a full complement of neighbours, as they would in a fully atomistic model will be referred to as free atoms. Atoms which do not have a full complement of neighbours are coloured green in figure 5.12 and will be referred to as imaginary atoms. These imaginary atoms exist to provide the neighbours of free atoms near the interface, and therefore, the size of the transition region and number of layers of imaginary atoms depends on the cut-off distance of the potential function.

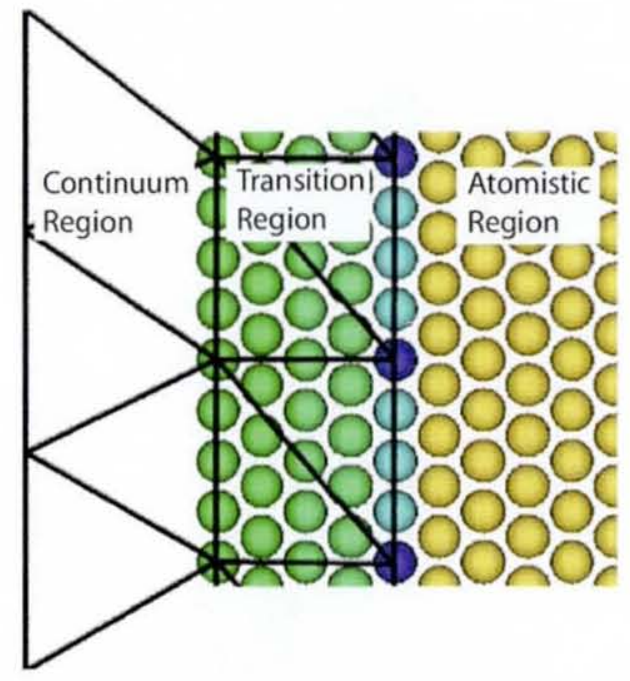

Figure 5.12: Schematic of the 2D generic coupled interface. The green imaginary atoms are contained within the first layer of elements and provide neighbours of the atoms modelled by MD.

The meshing of the continuum region near the interface is made coarse 
enough that all imaginary atoms are contained within the first layer of elements. The nodes on the inside layer must coincide with free atoms as these will be used to pass information from the atomistic region to the continuum region. The atoms which coincide with these nodes will be called node-atoms and are coloured dark blue in figure 5.12. There are free atoms which have a full complement of neighbours that lie on the boundary of the continuum region (coloured light blue in figure 5.12). Although these atoms are modelled in the same way as all other free atoms, they will be labelled separately as face atoms and will be used to analyse the method later.

The node-atoms are a subset of the free atoms of the system and hence have their positions calculated as a particle of a molecular dynamics simulation, via an atomistic potential function. The procedure to pass information to the continuum region about distortions in the atomistic region is then as follows. The node-atoms are in their correct positions according to the non-local atomistic description. The positions of the corresponding nodes are moved to match the positions of the atoms, giving a distortion to the FE model and hence a resulting force on other nodes. The dynamical solution in the continuum region is then advanced via the velocity Verlet algorithm.

To complete the coupling of the models, a feedback mechanism is required to inform the atomistic region of the distortion in the continuum region. For the method described here, this is achieved by a linear interpolation procedure based on the displacements of nodes of the finite elements. The imaginary atoms each have a fractional coordinate $(\xi, \eta)$ within an element at the edge of the continuum region. After the element has been distorted due to the position matching of node-atoms and subsequent dynamical FE response, the internal imaginary atoms are repositioned such that the fractional coordinates are kept constant within the deformed element. 
The set of equations 2.41 can be used to describe the $x$ and $y$ coordinates of a point $\left(x_{p}, y_{p}\right)$ within an element in terms of the point's fractional coordinates $\left(\xi_{p}, \eta_{p}\right)$ and the element's nodal positions. Rearranging this for the fractional coordinates for an imaginary atom within an element in terms of its cartesian coordinates gives

$$
\begin{aligned}
\xi_{p} & =\frac{\alpha_{\xi} x_{p}+\beta_{\xi} y_{p}+\gamma_{\xi}}{\Delta} \\
\eta_{p} & =\frac{\alpha_{\eta} x_{p}+\beta_{\eta} y_{p}+\gamma_{\eta}}{\Delta}
\end{aligned}
$$

where the coefficients are given in terms of the elements nodal positions as

$$
\begin{array}{r}
\alpha_{\xi}=x_{23}, \beta_{\xi}=-y_{23} \\
\gamma_{\xi}=y_{23} x_{3}-x_{23} y_{3} \\
\alpha_{\eta}=-x_{13}, \beta_{\eta}=y_{13} \\
\gamma_{\eta}=y_{3} x_{13}-y_{13} x_{3}
\end{array}
$$

and

$$
\Delta=-y_{23} x_{13}+x_{23} y_{13} .
$$

Because the fractional coordinates of imaginary atoms are kept fixed during a simulation, they are calculated once at the beginning of the simulation and stored so that they do not need to be recalculated at each step. The displacement $\left(u_{p}, v_{p}\right)$ of an imaginary atom is calculated using the set of equations 2.42, its fractional coordinates and the nodal displacements of the containing element.

$$
\begin{aligned}
& u_{p}=u_{13} \xi_{p}+u_{23} \eta_{p}+u_{3} \\
& v_{p}=v_{13} \xi_{p}+v_{23} \eta_{p}+v_{3} .
\end{aligned}
$$

These displacements are applied to the imaginary atoms after each FE time step so that the atom positions are correct before the next MD force calculation. 


\section{Assigning Imaginary Atoms to Elements}

The above method for positioning imaginary atoms according to element distortions assumes that it is known which element an atom lies within. Therefore, a technique is required for determining this for each imaginary atom in the transition region. The meshing algorithm described in section 5.1 tessellates the continuum region entirely with triangular elements, i.e. the $2 \mathrm{D}$ simplex.

The 2D simplex [47], $\sigma_{2}$, with 3 vertices $P_{1}\left(x_{1}, y_{1}\right), P_{2}\left(x_{2}, y_{2}\right)$ and $P_{3}\left(x_{3}, y_{3}\right)$, is defined as the set of points given by $\sum_{i=1}^{3} \lambda_{i} P_{i}$, where the $\lambda_{i}$ are real numbers such that $\lambda_{i} \geq 0$ and $\sum_{i=1}^{3} \lambda_{i}=1$. Hence to determine if a point $p\left(x_{p}, y_{p}\right)$ lies within the triangle with these vertices, the set of simultaneous equations

$$
\begin{aligned}
\lambda_{1} x_{1}+\lambda_{2} x_{2}+\lambda_{3} x_{3} & =x_{p} \\
\lambda_{1} y_{1}+\lambda_{2} y_{2}+\lambda_{3} y_{3} & =y_{p} \\
\lambda_{1}+\lambda_{2}+\lambda_{3} & =1
\end{aligned}
$$

are solved for the $\lambda_{i}$. The point lies in the triangle if $\lambda_{i} \geq 0$ for all $i$, and since the fractional coordinates remain constant, the imaginary atom stays in that triangle regardless of distortion. Hence the procedure for assigning imaginary atoms to elements needs to be performed only once at the beginning of the simulation.

\subsubsection{Testing and Results}

The position matching coupling method described above is designed to allow the interaction between an atomistic region and a continuum region, such that the phenomena due to a disturbance initiated in the atomistic region may pass into the continuum region or vice versa. To test this coupling 
method two test cases have been considered, quasi-static strain field propagation and elastic wave propagation. For both cases the Lennard-Jones potential (see section 2.2.1) was used to describe the interatomic forces in the atomistic region, and due to the stability of this potential under the high deformation in these test cases, the atoms were allowed only to move in the plane of the 2D continuum region (the $x$ and $y$ directions), and were arranged in the 2D close-packed lattice structure. The initial system setup and system parameters were the same for both simulations and can be seen in table 5.4.

\begin{tabular}{c|c}
\hline Parameter & Value \\
\hline \hline Atoms & $10,151\left(250 \times 217 \AA^{2}\right)$ \\
Inside edge nodes & 25 \\
Outside edge nodes & 8 \\
Total nodes & $3,080\left(2500 \times 2165 \AA^{2}\right)$ \\
Elements & 5,944 \\
Time step & $1 \mathrm{fs}$
\end{tabular}

Table 5.4: Parameters used for the 2D strain field and elastic wave propagation simulations.

In quasi-static materials experiments such as nanoindentation, the material experiences a long range strain field. To recreate this phenomenon in the $2 \mathrm{D}$ coupled model, atoms initially within $64 \AA$ of the centre of the atomistic region were moved outwards to a maximum of $5 \%$ of their original distance from the centre at a rate of $10 \mathrm{~m} / \mathrm{s}$. These atoms were then held fixed in position to continue to analyse the response in the surrounding material and the transition of the strain field from the atomistic region to the continuum region. Images of the $2 \mathrm{D}$ simulation at various time steps are shown in figure 
5.13. These images show the strain field crossing the interface smoothly and without reflection. The same hexagonal shape of the strain field (due to the 2D close-packed structure of the lattice) is seen to be transfered into the continuum region.
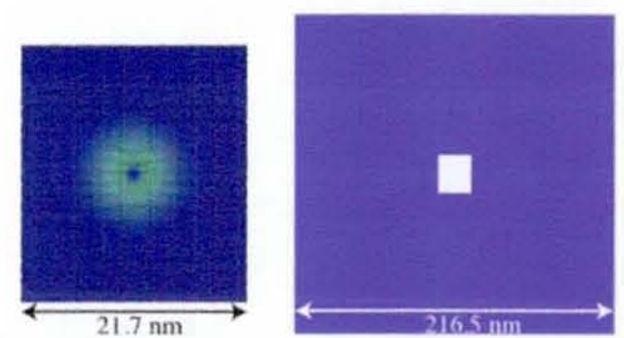

(a) $8 \mathrm{ps}$
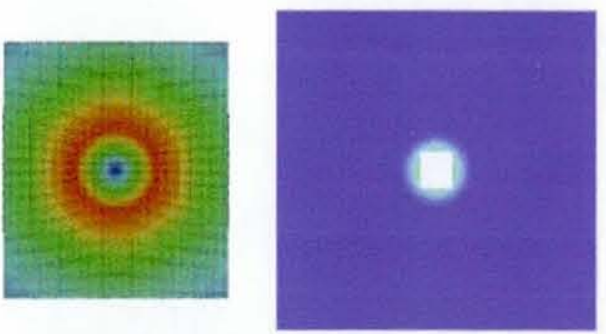

(c) $24 \mathrm{ps}$
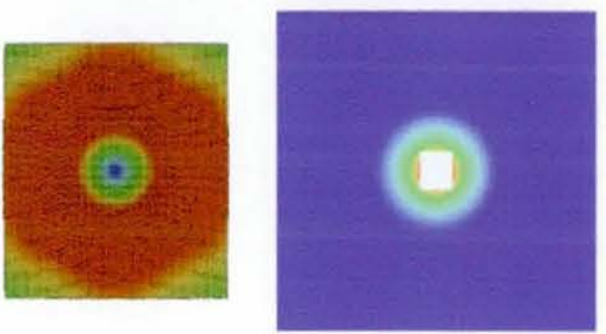

(e) $40 \mathrm{ps}$
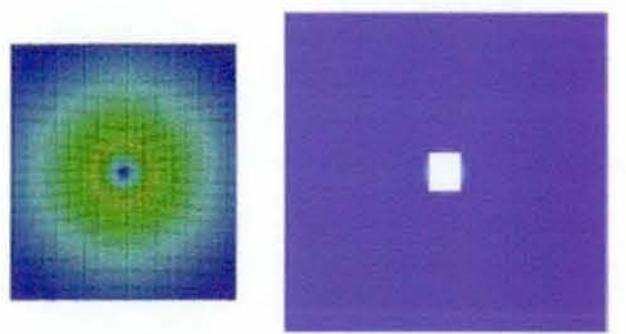

(b) $16 \mathrm{ps}$
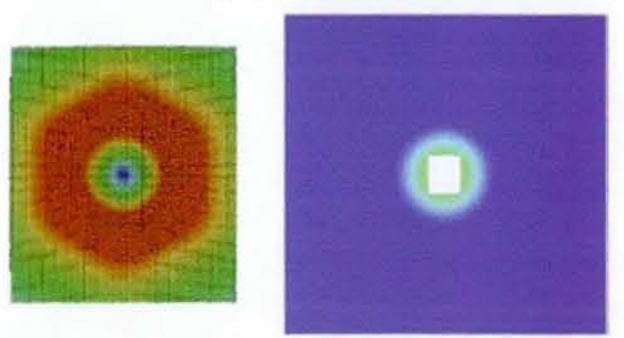

(d) $32 \mathrm{ps}$
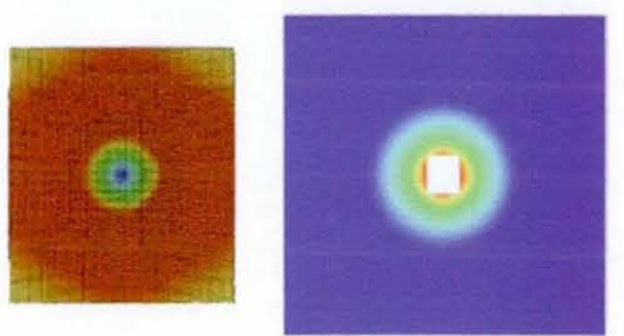

(f) $48 \mathrm{ps}$

Figure 5.13: Images from the strain test simulation taken at 8ps intervals. Both the atomistic and continuum regions are shown on the left and right of each figure respectively and are coloured according to displacement from zero (blue) to $0.2 \AA$ (red). The left hand image is the expanded version from the square in the centre of the right hand image. 
The induced distortion of the atomistic region stops after 40ps and the strain field in that region remains much the same after around 48ps, hence figure 5.14 shows images of only the continuum region after this time. These images show the long range strain field generated in the atomistic region continue to spread into the far field of the continuum region.

The identical distortion of the atomistic region was applied to an atomistic region with fixed boundary conditions to investigate the effect of the embedding continuum region. Images of the atomistic simulation are shown in figure 5.15 taken at the same times as those in figure 5.13 to give a comparison of the two simulations. Obviously the fixed boundary conditions prevent the strain field from passing the edge of the atomistic region, an unnatural restriction on the simulation as shown by the results from the coupled model. Although difficult to visualise from the static images, the fixed boundary conditions also generate reflected waves which continue to disturb the atomistic region for the remainder of the simulation, unlike the static atomistic region in the coupled model after the initial $48 \mathrm{ps}$.

Figure 5.16 shows the energy in the atomistic and continuum regions along with the total system energy for the coupled model. During the first 40ps the energy increases in the atomistic region as the material in this region is expanded. Due to the coupling method, the strain field is passed into the continuum region and the energy also increases in this region as expected. The expansion of the atomistic region stops after 40ps and the total system energy is conserved for the remaining time of the simulation. There is, however a short period of about 8ps where the strain field continues to spread from the atomistic region into the continuum region, which is illustrated by the drop in energy in the atomistic region and a continued increase in energy in the continuum region. 


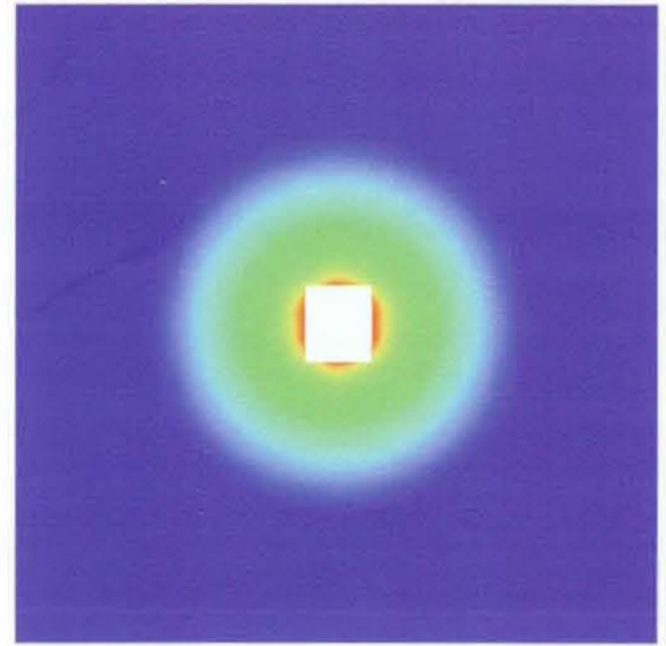

(a) $60 \mathrm{ps}$

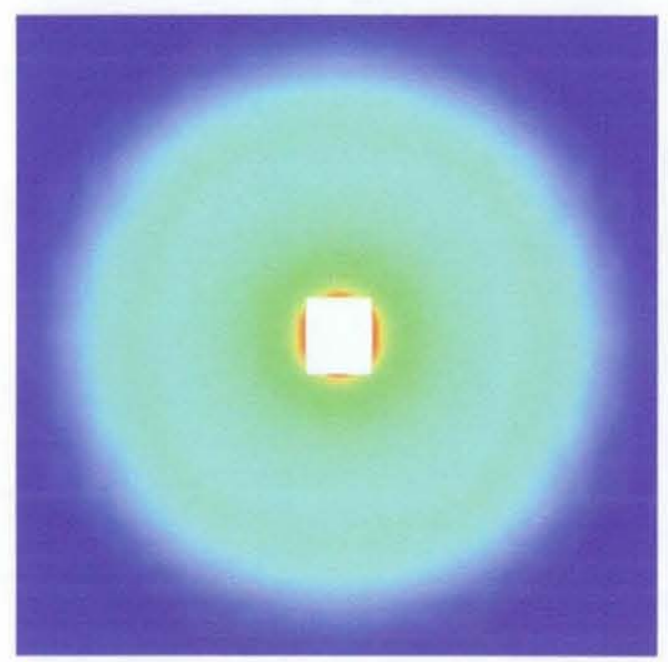

(c) $100 \mathrm{ps}$

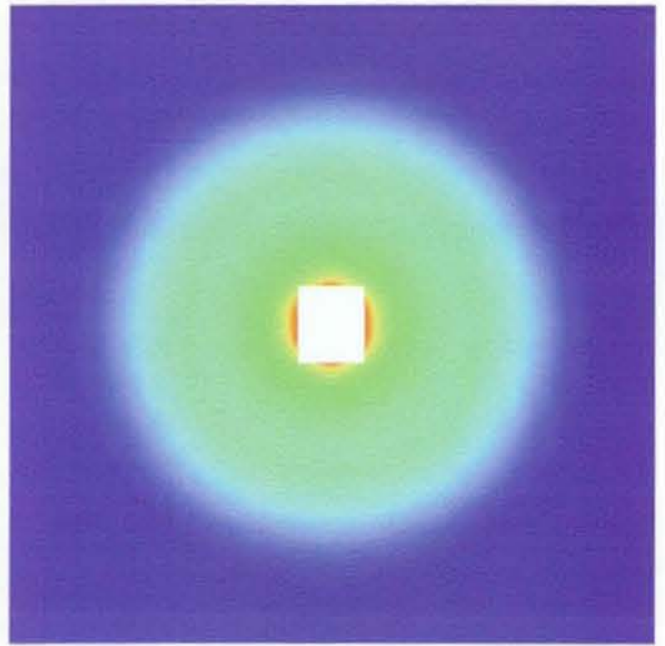

(b) $80 \mathrm{ps}$

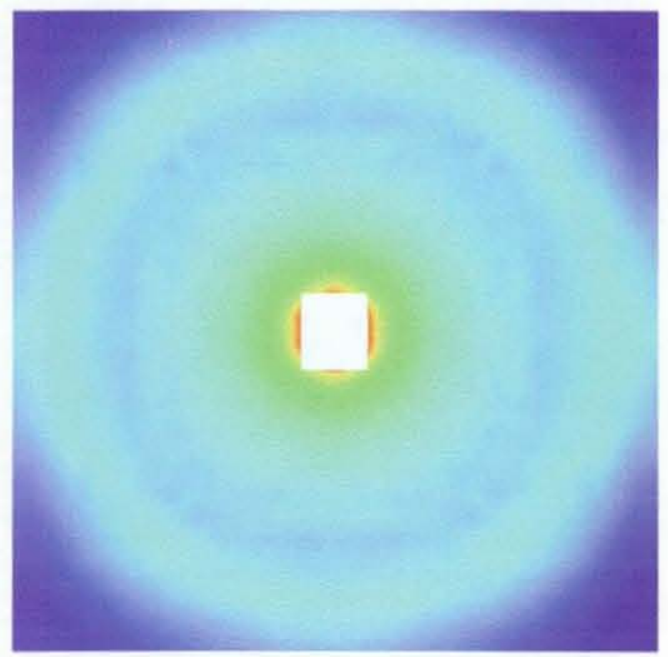

(d) $120 \mathrm{ps}$

Figure 5.14: Images of the continuum region from the strain test simulation taken at 20 ps intervals.

The comparison of the total system energy in the coupled model and the atomistic model with fixed boundary conditions is shown in figure 5.17. A considerably larger energy is needed to produce the same distortion of the atomistic region in the atomistic only simulation. This is due to the 


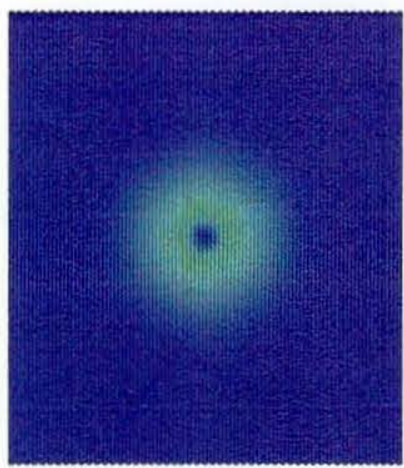

(a) $8 \mathrm{ps}$

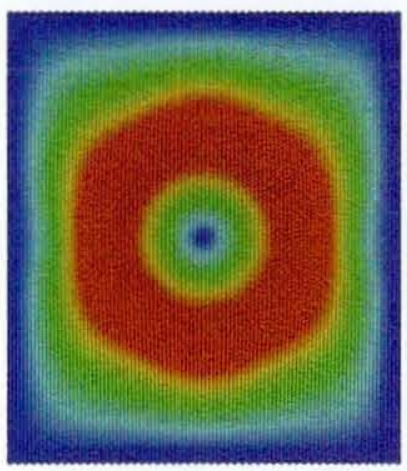

(d) $32 \mathrm{ps}$

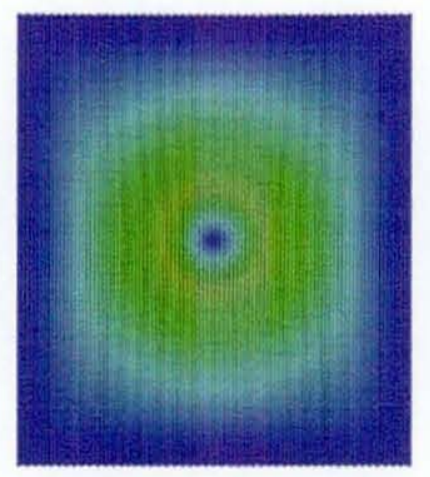

(b) $16 \mathrm{ps}$

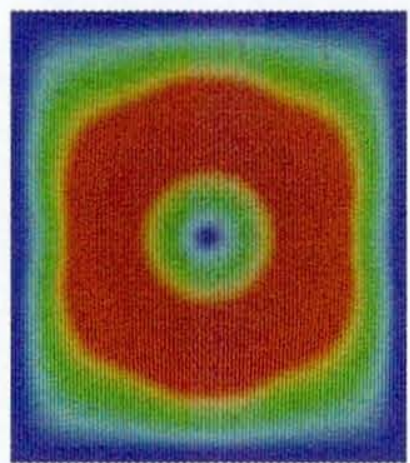

(e) $40 \mathrm{ps}$

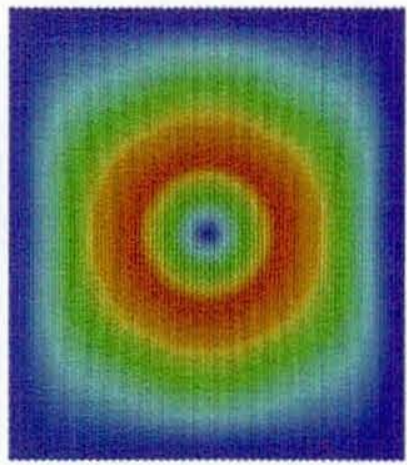

(c) $24 \mathrm{ps}$

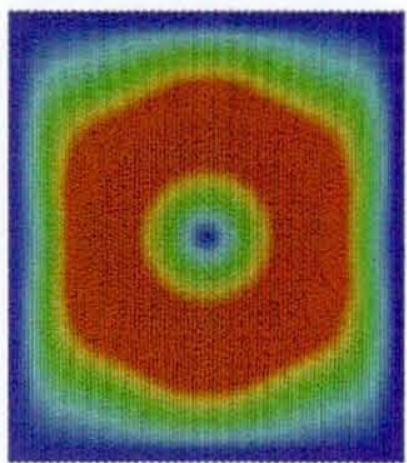

(f) $48 \mathrm{ps}$

Figure 5.15: Images from the strain test simulation with fixed boundary conditions taken at $8 \mathrm{ps}$ intervals. The fixed boundaries prevent the strain field from spreading into the far field.

fixed boundary conditions producing an artificially high resistance to the distortion.

The face atoms defined in section 5.5.1 are a subset of the free atoms in the system. As such, these atoms have a full complement of neighbours and are modelled entirely by the MD model. However, these face atoms lie on the face of an element of the continuum region and the position interpolation procedure used to reposition the imaginary atoms can be used to give a repositioning of the face atoms. The displacement of one particular face 


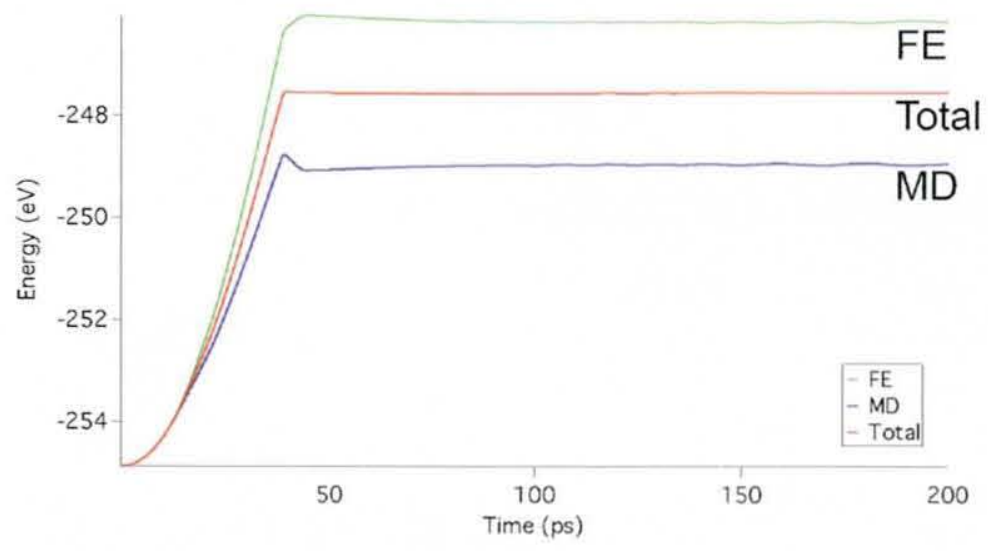

Figure 5.16: The energy in the atomistic region (blue), the continuum region (green) and the total system energy (red) for the coupled strain field propagation simulation.

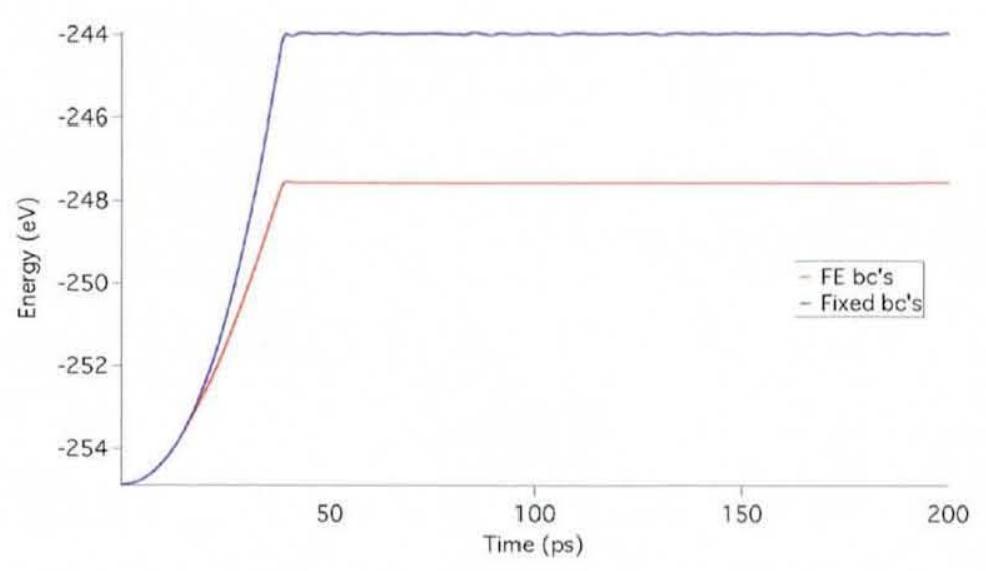

Figure 5.17: A comparison of the total system energies for the coupled model (red) and the atomistics only model (blue).

atom is shown in figure 5.18 along with the displacement of the same atom calculated by the interpolation procedure. This gives an indication of the accuracy of the interpolation approximation at each step, which is shown by figure 5.18 to be perfect for the $2 \mathrm{D}$ strain test case as there is no difference 
between the displacements calculated by the two models.

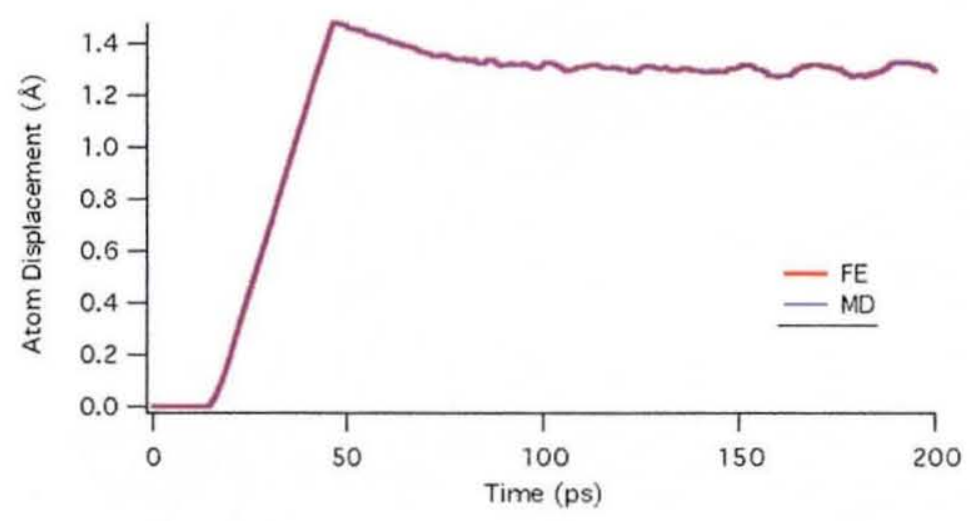

Figure 5.18: The displacement of a face atom during the 2D strain propagation test simulation, calculated by atomistics (blue) and continuum mechanics (red).

In order to test the ability of the coupled model to transmit elastic waves generated in an atomistic region into a continuum region, a second test case has been examined. The same initial system was used as in the strain field simulation, however, instead of slowly moving atoms apart to induce a strain field, the atoms within $25 \AA$ of the centre of the atomistic region were drawn towards the centre during the initial 10ps and were subsequently released to generate elastic waves that propagate into the continuum region over the next 90 ps. Images of the simulation are shown in figures 5.19 and 5.20, of the atomistic and continuum regions respectively. After 16ps the elastic waves begin to move from the atomistic region into the continuum region, resulting in a small reflection of the waves back into the atomistic region. One of the main objectives of adding the embedding continuum region is to remove reflection of waves which interfere with the simulated experiment. To see how effective the coupling method is at removing the reflection, a 


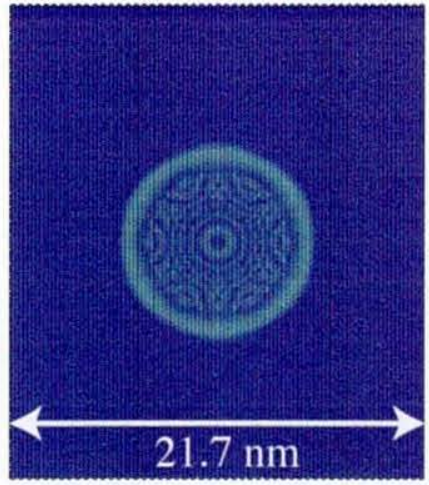

(a) $10 \mathrm{ps}$

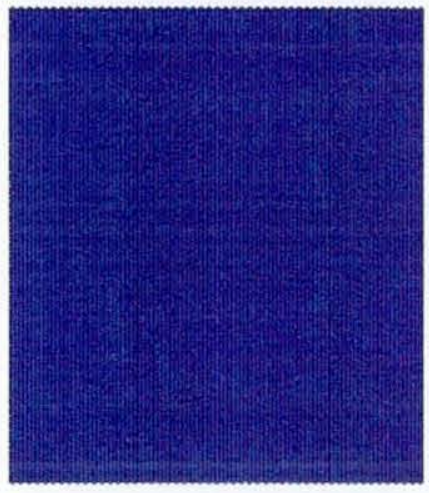

(d) $40 \mathrm{ps}$

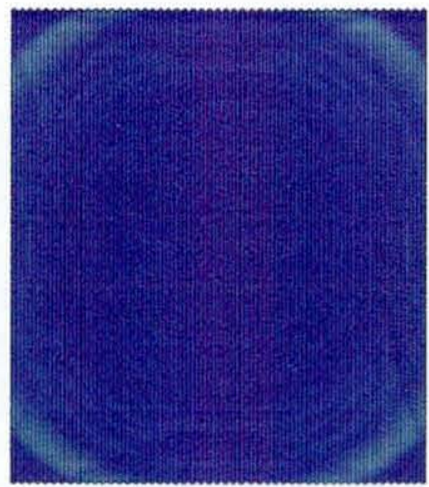

(b) $20 \mathrm{ps}$

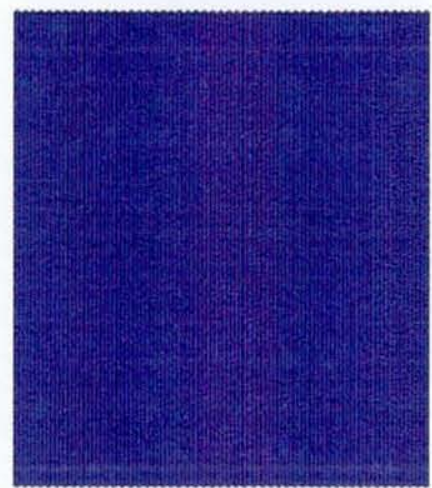

(e) $50 \mathrm{ps}$

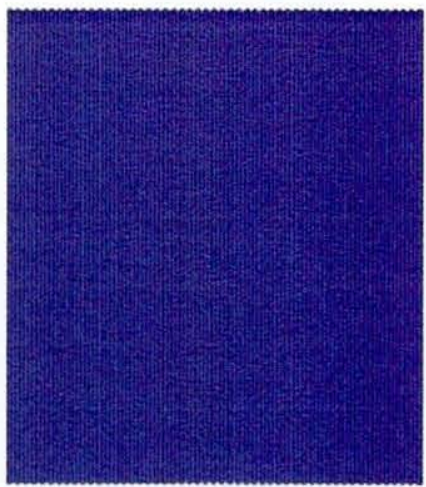

(c) $30 \mathrm{ps}$

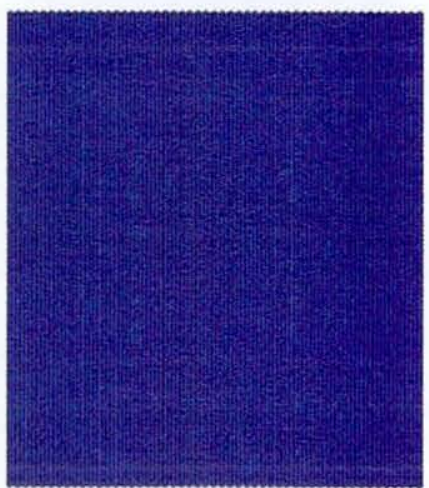

(f) $60 \mathrm{ps}$

Figure 5.19: Images of the atomistic region from the elastic wave test simulation taken at 10 ps intervals. There is very little disturbance after the waves have entered the continuum region.

comparative simulation has been performed with the same wave generation, but with fixed boundary conditions around the atomistic region. Images of this simulation are shown in figure 5.21.

Without the continuum region to transmit disturbances into the far field, the elastic waves are totally reflected back into the atomistic region. When compared to the images in figure 5.19 of the atomistic region from the coupled model, it can be clearly seen that the reflection is significantly reduced by 


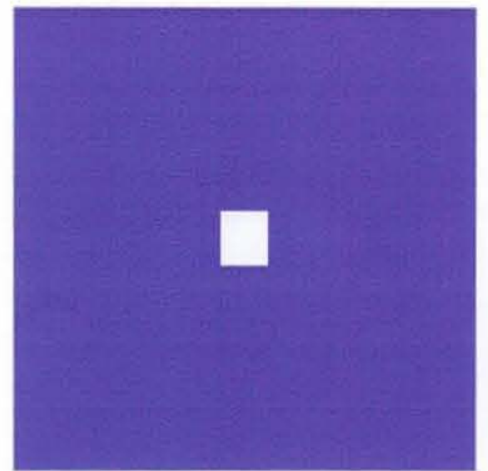

(a) $10 \mathrm{ps}$

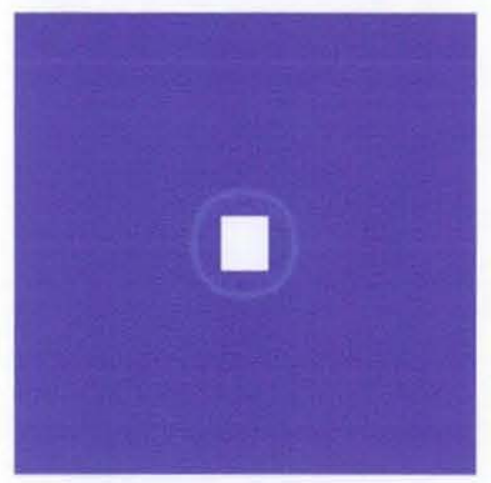

(c) $30 \mathrm{ps}$

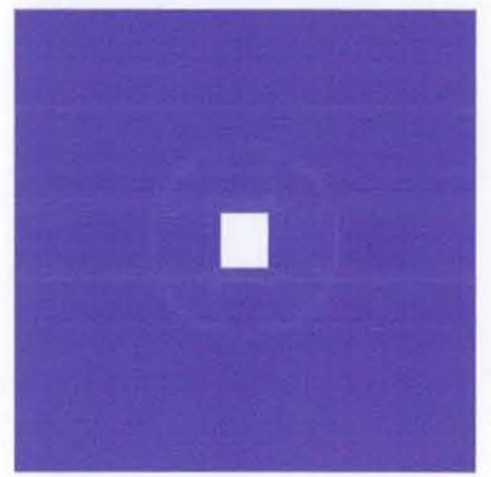

(e) $50 \mathrm{ps}$

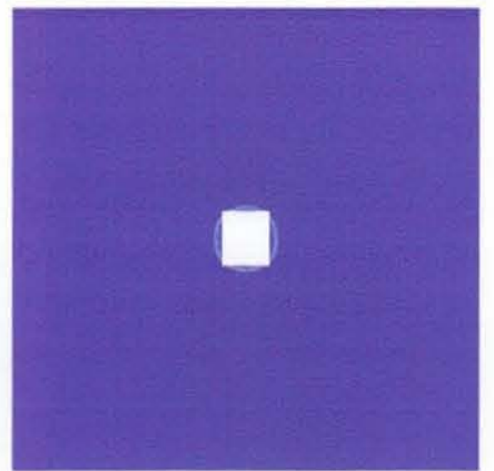

(b) $20 \mathrm{ps}$

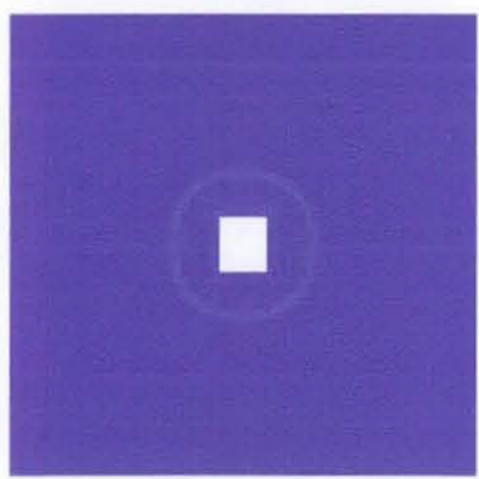

(d) 40ps

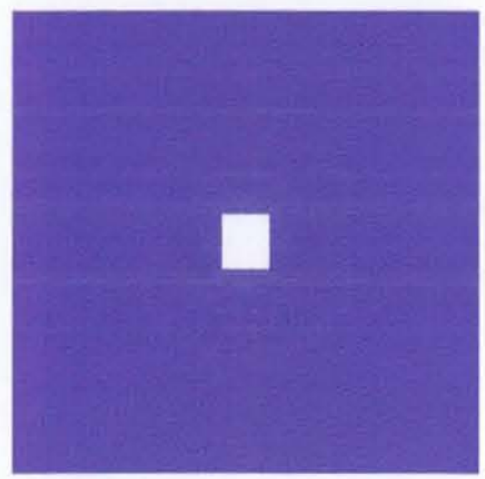

(f) $60 \mathrm{ps}$

Figure 5.20: Images of the continuum region from the elastic wave test simulation taken at $10 \mathrm{ps}$ intervals. The elastic wave generated in the atomistic region moves smoothly into the continuum region. 


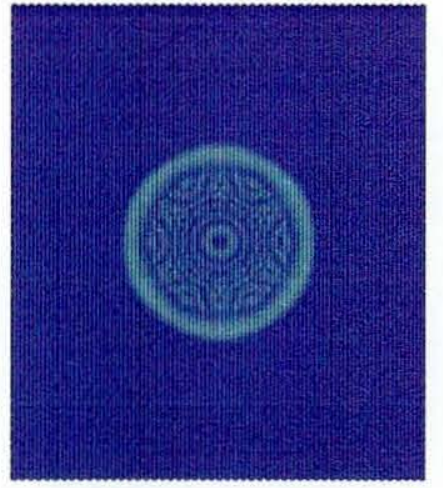

(a) $10 \mathrm{ps}$

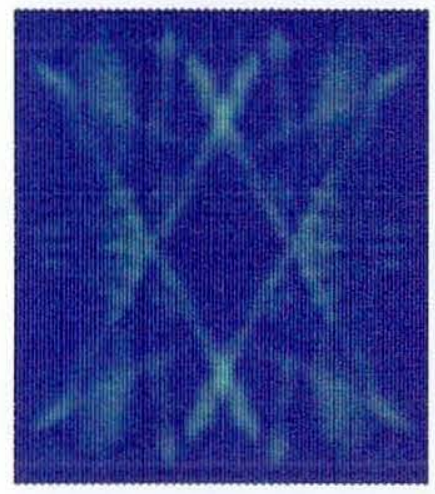

(d) $40 \mathrm{ps}$

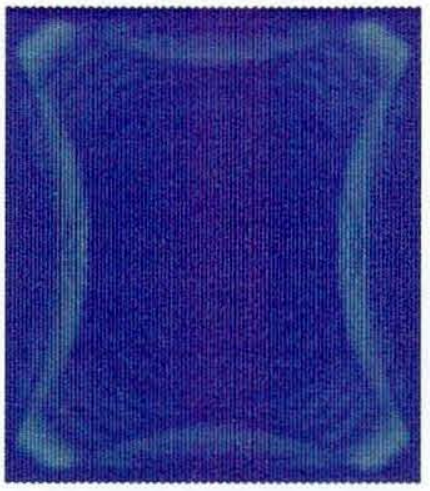

(b) $20 \mathrm{ps}$

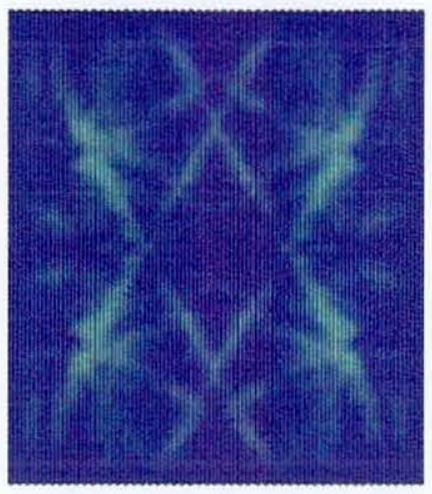

(e) $50 \mathrm{ps}$

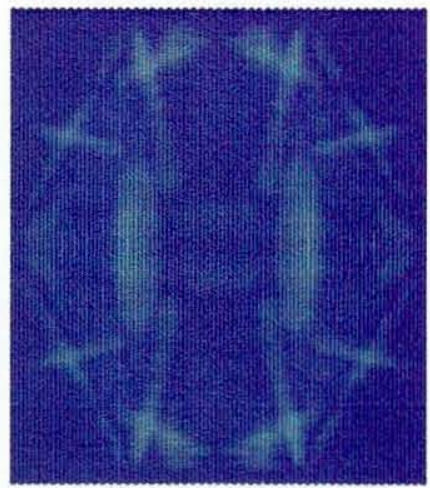

(c) $30 \mathrm{ps}$

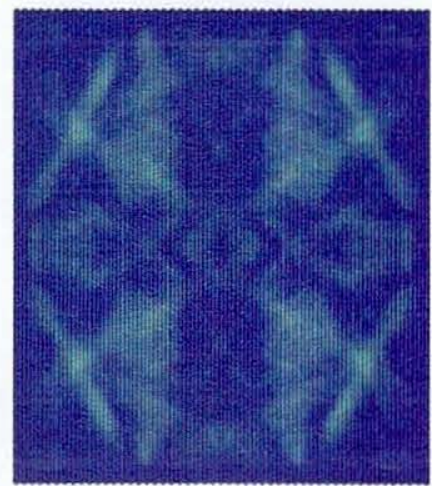

(f) $60 \mathrm{ps}$

Figure 5.21: Images of the atomistic region from the elastic wave test simulation with fixed boundary conditions. The elastic waves are reflected back into the atomistic region and result in interference waves.

the addition of the embedding continuum region.

\subsubsection{Conclusions}

The results from the two test simulations have shown that the $2 \mathrm{D}$ position matching coupling technique can be used to model long range phenomena such as strain fields and elastic wave propagation in the far field. In both cases, the unnatural reflection observed when fixed boundaries are applied 
to atomistic simulations is almost entirely removed. The removal of these reflected waves from 3D simulations of material deformation would prevent unrealistic interference with nanoscale phenomenon. Furthermore, in the strain field simulation the energy required to distort the material was significantly lower than in the atomistic only simulation due to the artificial hardening by the fixed boundaries. This could make a significant difference to material properties calculated from simulation results. Unlike the initial model described in section 5.4, the position matching coupling technique could work with many-body potentials where the concept of an interatomic force $F_{i j}$ does not exist. Therefore, in the next chapter, this method will be extended to $3 \mathrm{D}$ so that it may be applied to real experiments such as nanoindentation. 


\section{Chapter 6}

\section{Three Dimensional Multiscale}

\section{Modelling}

The work in this chapter follows from the development work of chapter 5 . The position matching coupling technique in 2D (described in section 5.5) allows long range effects of nanoscale distortions to be modelled in the far field by continuum mechanics. However, modelling realistic scientific experiments requires a $3 \mathrm{D}$ model to replicate accurately the experiment and hence, the position matching technique has been extended to 3D. As well as describing the extension of the position matching technique to $3 \mathrm{D}$, this chapter also describes another coupling technique with an improved method for dealing with interface interactions.

When implementing a 3D multiscale model, the coupling technique is not the only problem to be addressed. The tessellation of the embedding continuum region in a manner suitable for coupling to an atomistic region is by no means a trivial exercise, and like in the $2 \mathrm{D}$ case, the elastic properties of the non-local atomistic interactions must match the elastic properties of the local continuum interactions. Both of these issues are addressed in this 
chapter.

\subsection{Meshing Technique for Embedding Atom- istics in $3 \mathrm{D}$}

The multiscale models described in this section couple an atomistic model to a continuum region modelled by a $3 \mathrm{D}$ linear elastic FE method. Therefore, a method is described here to tessellate a continuum region suitable for embedding an atomistic region to model nanoindentation experiments.

The process begins by generating five cuboidal pillars positioned around an empty cube (which will be filled by the atomistic region) as shown in figure 6.1. All of the pillars have the same dimensions and are made up of smaller cubes which in turn are tessellated into 6 tetrahedra as this gives elements of equal volume [48]. The cubiods are to be deformed to fill the space between them (the blue box in figure 6.1) and hence to avoid creating highly irregular tetrahedra, the orientation of the cube's tessellation depends on which quarter of the square cross-section of the cuboid it lies within. The pillars labelled a-d are stretched so that the outside square faces match the corresponding blue rectangle on the boundary of the continuum region, as illustrated for pillar a in figure 6.2(a). Pillar e is stretched so that the square on its bottom surface matches the corresponding blue square on the bottom surface of the continuum region as illustrated in figure 6.2(b). The nodes of the tetrahedral elements are repositioned linearly within the pillar such that their fractional positions remain constant.

Once the space has been filled, repeated nodes where the deformed pillars meet are removed and the layers of nodes in the outer regions for the mesh may be stretched even further to reduce the node density in the far field and 


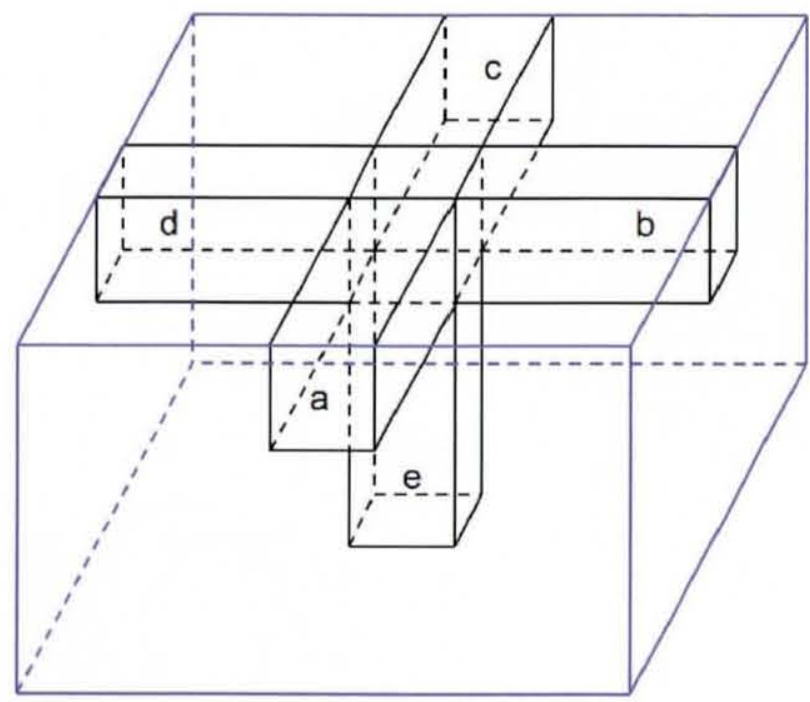

Figure 6.1: A schematic diagram of the initial pillars used to tessellate the continuum region.

model larger volumes of material with the same computational expense.

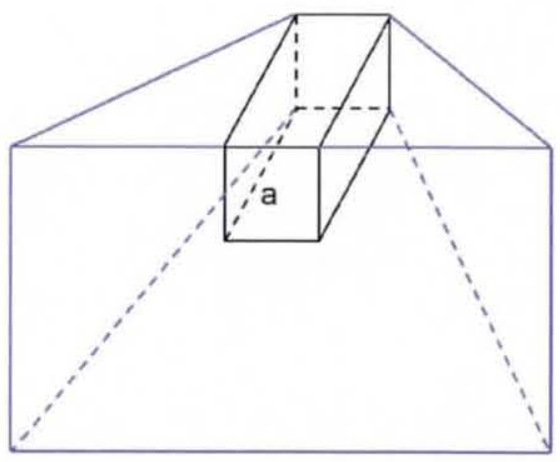

(a)

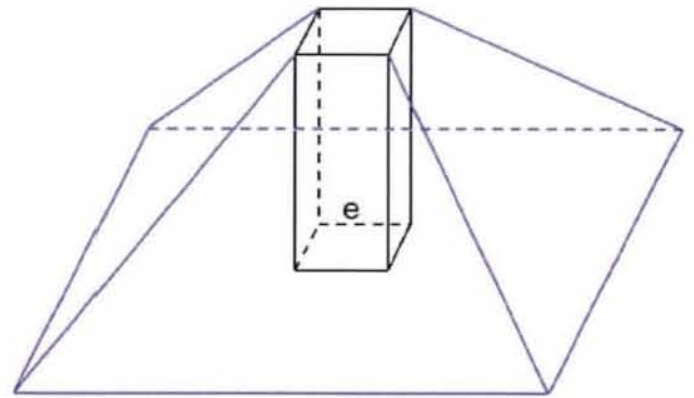

(b)

Figure 6.2: The cuboidal pillars are stretched to fill the space between them. (a) shows the final shape of pillar a which is also used for pillars b-d. (b) shows the final shape of pillar e. 


\subsection{Matching Elastic Constants in 3D}

Molecular dynamics simulations calculate interatomic forces via empirical potential functions such as those described in section 2.2. The potential functions used to describe real solid materials are fitted to empirical data including the elastic constants $C_{11}, C_{12}$ and the shear modulus $C_{44}$. The 3D finite element model for stress analysis described in section 2.3 .2 requires the Young's modulus and the Poisson's ratio to describe the elastic response of the material under investigation. By considering the relationships between the Young's modulus, $E$, bulk modulus, $B$, and shear modulus for isotropic materials in equations 6.1 and 6.2 , and noting that the bulk modulus is related to the elastic constants by $B=\left(C_{11}+2 C_{12}\right) / 3$, the parameters required for the FE model can be obtained from the empirical data used to fit the potential function.

$$
\begin{gathered}
B=\frac{E}{3(1-2 \nu)} \\
C_{44}=\frac{E}{2(1+\nu)}
\end{gathered}
$$

Eliminating $B$ and rearranging for $E$ and $\nu$ gives the following expressions for the FE parameters.

$$
\begin{aligned}
& E=\frac{3 C_{11} C_{44}+6 C_{12} C_{44}}{C_{11}+2 C_{12}+C_{44}} \\
& \nu=\frac{C_{11}+2 C_{12}-2 C_{44}}{2 C_{11}+4 C_{12}+2 C_{44}}
\end{aligned}
$$

The Ackland potential for $\mathrm{Au}[11]$ and $\mathrm{Fe}[12]$ are fitted to the elastic constants in table 6.1. The above expressions have been used to calculate the Young's modulus and Poisson's ratio, also shown in table 6.1, which have been used in the multiscale simulations in chapter 7 . 


\begin{tabular}{c||ccc|cc} 
& $C_{11}$ & $C_{12}$ & $C_{44}$ & $E$ & $\nu$ \\
\hline \hline & $(\mathrm{GPa})$ & $(\mathrm{GPa})$ & $(\mathrm{GPa})$ & $(\mathrm{GPa})$ & \\
\hline $\mathrm{Au}$ & 186.0 & 157.0 & 42.0 & 116.2 & 0.384 \\
$\mathrm{Fe}$ & 243.0 & 137.9 & 114.7 & 282.0 & 0.228
\end{tabular}

Table 6.1: The elastic constants $C_{11}, C_{12}$ and the shear modulus $C_{44}$ are used to fit the potential function and to calculate the Young's modulus, E, and Poisson's ratio, $\nu$ for the FE model. This ensures that the elastic properties in both the atomistic and continuum regions of a multiscale model are matched.

\subsection{Assigning Imaginary Atoms to Elements}

For the 3D multiscale models described in this chapter, the methodologies require a technique to determine which element each imaginary atom in the transition region lies within. The meshing algorithm described in section 6.1 tessellates the continuum region entirely with tetrahedral elements, i.e. the $3 \mathrm{D}$ simplex. Hence, the method for assigning atoms to $2 \mathrm{D}$ triangular elements (the 2D simplex) in section 5.5.1 is extended here to 3D.

The $3 \mathrm{D}$ simplex [47], $\sigma_{3}$, with 4 vertices at the points $P_{1}\left(x_{1}, y_{1}, z_{1}\right)$, $P_{2}\left(x_{2}, y_{2}, z_{2}\right), P_{3}\left(x_{3}, y_{3}, z_{3}\right)$ and $P_{4}\left(x_{4}, y_{4}, z_{4}\right)$, is defined as the set of points $\sum_{i=1}^{4} \lambda_{i} P_{i}$, where the $\lambda_{i}$ are real numbers such that $\lambda_{i} \geq 0$ and $\sum_{i=1}^{4} \lambda_{i}=1$. Hence to determine if a point $p\left(x_{p}, y_{p}, z_{p}\right)$ lies within the tetrahedron with these verticies, the set of simultaneous equations

$$
\begin{aligned}
\lambda_{1} x_{1}+\lambda_{2} x_{2}+\lambda_{3} x_{3}+\lambda_{4} x_{4} & =x_{p} \\
\lambda_{1} y_{1}+\lambda_{2} y_{2}+\lambda_{3} y_{3}+\lambda_{4} y_{4} & =y_{p} \\
\lambda_{1} z_{1}+\lambda_{2} z_{2}+\lambda_{3} z_{3}+\lambda_{4} z_{4} & =z_{p} \\
\lambda_{1}+\lambda_{2}+\lambda_{3}+\lambda_{4} & =1
\end{aligned}
$$


are solved for the $\lambda_{i}$. The point lies within the tetrahedron if $\lambda_{i} \geq 0$ for all $i$, and since the fractional coordinates remain constant, the imaginary atom stays on that tetrahedron regardless of distortion. Hence the procedure for assigning imaginary atoms to elements needs to be performed only once at the beginning of the simulation.

\subsection{Position Matching Generic Coupling Tech- nique in $3 \mathrm{D}$}

The position matching technique described in section 5.5 is extended to a fully 3D model. The system is modelled by embedding an atomistic region in the centre of a continuum region with coupling along the boundaries. Figure 6.3 illustrates schematically the geometry involved. The atomistic region is positioned at the top of the continuum region so that it has a free surface. The dynamical processes due to experiments (such as nanoindentation or laser ablation) are assumed to be initiated at the free surface with their effect transmitted to the continuum region as the disturbance spreads.

\subsubsection{Methodology}

As in the $2 \mathrm{D}$ position matching method, a transition region is defined where the atomistic and continuum regions overlap, and it is this transition region that allows the non-local atomistic description to local continuum description transformation to occur. Imaginary atoms exist at regular lattice positions within the transition region, acting as neighbours of the free atoms in the atomistic region. Hence, the thickness of the transition region is governed by the cut-off distance of the potential function used in the MD model, as 


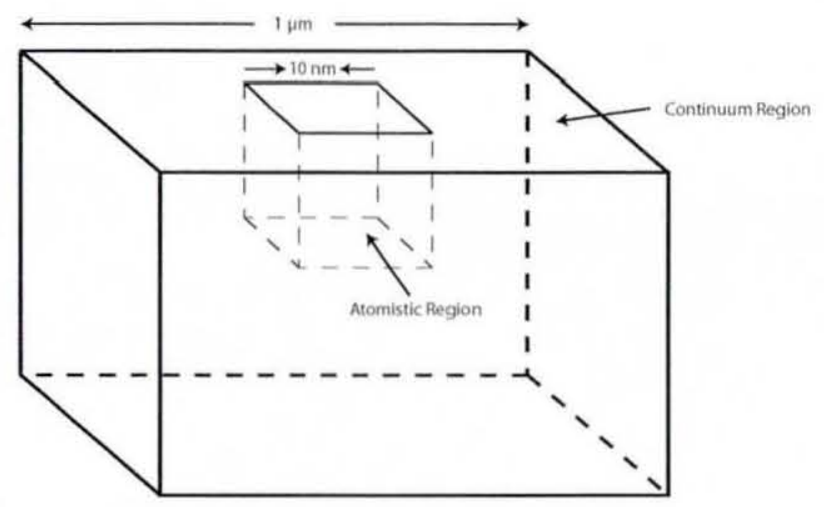

Figure 6.3: Embedding an atomistic region within a continuum region for simulating nanoindentation. Typical length scales are shown for the two regions.

there must be sufficient imaginary atoms to provide all free atoms with a full complement of neighbours. Figure 6.4(b) shows the atoms which exist in a typical transition region. The inside surfaces of the transition region are filled with dark blue node-atoms and light blue face atoms. Both of these types of atom are subsets of the free atoms of the coupled system, i.e. atoms which have a full complement of neighbours and are modelled entirely by MD. The node-atoms are placed on a regular grid and coincide with the FE nodes on the boundary of the mesh. As in the 2D model, the face atoms are used to test the coupling technique by comparing their actual positions due to the MD model to their interpolated position due to the FE model. The continuum and transition regions are tessellated with tetrahedra by the meshing algorithm described in section 6.1 . On the inside boundary with the atomistic region, the first layer of elements is made large enough so that it contains all of the imaginary atoms of the transition region. This is to ensure that the reaction of an imaginary atom due to the motion of a node atom occurs within the same time step. 


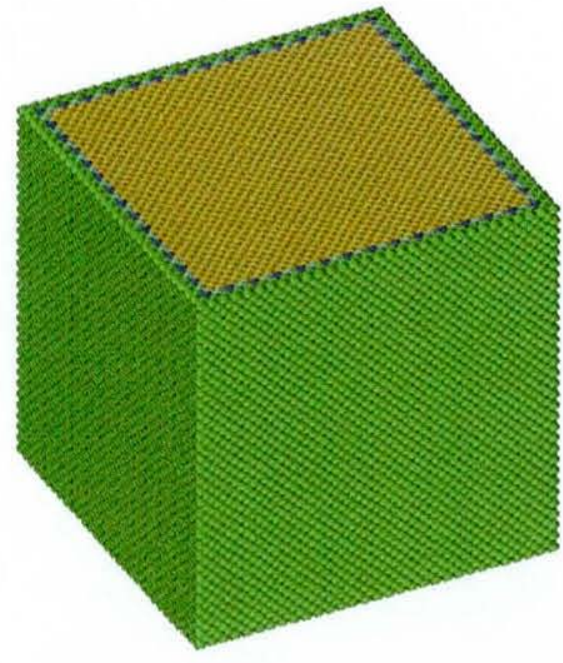

(a)

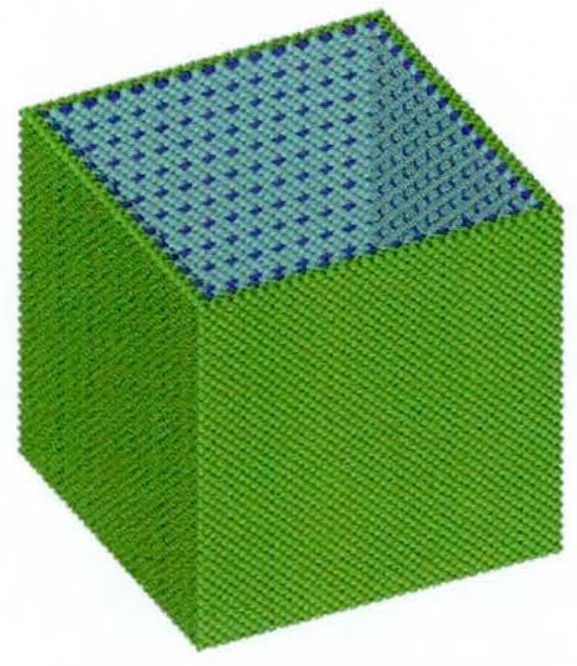

(b)

Figure 6.4: Atom types of the coupled model. In the atomistic region atoms are coloured yellow, imaginary atoms are coloured green, node-atoms are dark blue and face atoms are light blue. (a) shows all atoms of the coupled model. (b) shows only atoms within the transition region.

The node-atoms are used to pass information to the continuum region about distortions in the atomistic region. The node-atoms are in their correct positions according to the non-local atomistic description. The positions of the corresponding nodes are moved to match the positions of the atoms, giving a distortion to the FE model and hence a resulting force on other nodes. The dynamical solution in the continuum region is then advanced via the velocity Verlet algorithm.

A linear interpolation procedure based on the displacements of nodes of the finite elements is used to provide a feedback mechanism informing the atomistic region of the distortion in the continuum region. The imaginary atoms each have a fractional coordinate $(\xi, \eta, \zeta)$ within an element at the edge of the continuum region. After the element has been distorted due to 
the position matching of node-atoms and subsequent dynamical FE response, the internal imaginary atoms are repositioned such that the fractional coordinates are kept constant within the deformed tetrahedral element.

The set of equations 2.59 can be used to describe the $x, y$, and $z$ coordinates of a point $\mathrm{P}\left(x_{p}, y_{p}, z_{p}\right)$ within an element in terms of the points fractional coordinates $\left(\xi_{p}, \eta_{p}, \zeta_{p}\right)$ and the elements nodal positions. Rearranging this for the fractional coordinates for an imaginary atom within an element in terms of its cartesian coordinates gives

$$
\begin{aligned}
\xi_{p} & =\frac{\alpha_{\xi} x_{p}+\beta_{\xi} y_{p}+\gamma_{\xi} z_{p}+\delta_{\xi}}{\Delta} \\
\eta_{p} & =\frac{\alpha_{\eta} x_{p}+\beta_{\eta} y_{p}+\gamma_{\eta} z_{p}+\delta_{\eta}}{\Delta} \\
\zeta_{p} & =\frac{\alpha_{\zeta} x_{p}+\beta_{\zeta} y_{p}+\gamma_{\zeta} z_{p}+\delta_{\zeta}}{\Delta}
\end{aligned}
$$

where the coefficients are given in terms of the elements nodal positions as

$$
\begin{gathered}
\alpha_{\xi}=-z_{34} y_{24}+y_{34} z_{24}, \beta_{\xi}=-x_{34} z_{24}+z_{34} x_{24}, \gamma_{\xi}=-y_{34} x_{24}+x_{34} y_{24} \\
\delta_{\xi}=-y_{24} x_{34} z_{4}-y_{34} z_{24} x_{4}+y_{4} x_{34} z_{24}-y_{4} z_{34} x_{24}+y_{34} z_{4} x_{24}+y_{24} z_{34} x_{4} \\
\alpha_{\eta}=-z_{14} y_{34}+y_{14} z_{34}, \beta_{\eta}=x_{34} z_{14}-z_{34} x_{14}, \gamma_{\eta}=-y_{14} x_{34}+x_{14} y_{34} \\
\delta_{\eta}=y_{14} x_{34} z_{4}+z_{34} x_{14} y_{4}-y_{14} z_{34} x_{4}-x_{14} y_{34} z_{4}-x_{34} z_{14} y_{4}+z_{14} y_{34} x_{4} \\
\alpha_{\zeta}=-z_{24} y_{14}+y_{24} z_{14}, \beta_{\zeta}=x_{14} z_{24}-z_{14} x_{24}, \gamma_{\zeta}=-x_{14} y_{24}+y_{14} x_{24} \\
\delta_{\zeta}=-z_{14} y_{24} x_{4}+y_{14} z_{24} x_{4}-z_{24} x_{14} y_{4}-y_{14} x_{24} z_{4}+x_{14} y_{24} z_{4}+z_{14} x_{24} y_{4}
\end{gathered}
$$

and

$$
\begin{aligned}
\Delta= & x_{34} z_{14} y_{24}-z_{34} x_{14} y_{24}-y_{14} x_{34} z_{24}-z_{14} y_{34} x_{24} \\
& +x_{14} y_{34} z_{24}+y_{14} z_{34} x_{24}
\end{aligned}
$$

Because the fractional coordinates of imaginary atoms are kept fixed during a simulation, they are calculated once at the beginning of the simulation 
and stored so that they do not need to be recalculated at each step. The displacement $\left(u_{p}, v_{p}, w_{p}\right)$ of an imaginary atom is calculated using the set of equations 2.58, its fractional coordinates and the nodal displacements of the containing element.

$$
\begin{aligned}
u_{p} & =u_{14} \xi_{p}+u_{24} \eta_{p}+u_{34} \zeta_{p}+u_{4} \\
v_{p} & =v_{14} \xi_{p}+v_{24} \eta_{p}+v_{34} \zeta_{p}+v_{4} \\
w_{p} & =w_{14} \xi_{p}+w_{24} \eta_{p}+w_{34} \zeta_{p}+w_{4} .
\end{aligned}
$$

These displacements are applied to the imaginary atoms after each FE time step so that the atom positions are correct before the next MD force calculation.

\subsubsection{Testing and Results}

A nanoindentation simulation into a lattice with first nearest neighbour Lennard-Jones interactions has been performed to test the 3D position matching method. This simulation is used to investigate the passing of the strain field produced by the indentation, from the atomistic region into the continuum region. A similar technique to that described in section 5.4.2 is used to produce the indent. A paraboliod was moved at a velocity of $5 \mathrm{~m} / \mathrm{s}, 10 \AA$ into the top surface of the atomistic region over a period of $200 \mathrm{ps}$. At each time step, any atom within the paraboloid was moved directly downwards to the paraboloid's surface, thus creating an indentation. After 200 ps the indenter is held fixed for a further 50 ps to check that the total system energy is conserved.

The dimensions of the atomistic and continuum regions and the number of atoms and nodes in the respective regions are given in table 6.2. The equivalent total number of atoms in this table gives an approximate value of 
the number of atoms represented by the combined atomistic and continuum regions. Therefore, by assuming that moving a node forward in time is as equally computationally costly as moving an atom forward in time (which is extremely conservative for any realistic potential), the coupled model is in the region of 20 times faster than if this many atoms had been modelled by molecular dynamics alone.

\begin{tabular}{c|c}
\hline Parameter & Value \\
\hline \hline MD dimensions $\left(\AA^{3}\right)$ & $106 \times 106 \times 113$ \\
FE dimensions $\left(\AA^{3}\right)$ & $382 \times 382 \times 230$ \\
MD atoms & 117,212 \\
FE nodes & 29,464 \\
Equivalent total atoms & $\approx 3.1 \times 10^{6}$
\end{tabular}

Table 6.2: The system size for the 3D position matching method test simulation. 'Total atoms' is the approximate number of atoms represented by the combined atomistic and continuum regions.

Images of the simulation in figure 6.5 show the strain field caused by the indentation spreading throughout the material in 50 ps intervals. In each diagram the atomistic region is displayed to the left and has been taken from the gap in the continuum region to its right. The colouring is based on displacement, from zero (dark blue) to $5 \AA$ (red). The first three images of the simulation from 100 ps to 200 ps show that the strain field moves smoothly across the interface into the continuum region which models the long range effects of the indentation. However, between 200 ps and 250 ps, the indenter is held fixed, but figure 6.5(d) shows far greater displacements than in figure 6.5(c). The reason for this is apparent from the energy graph in figure 6.6. The total energy rises during the indentation period as expected 

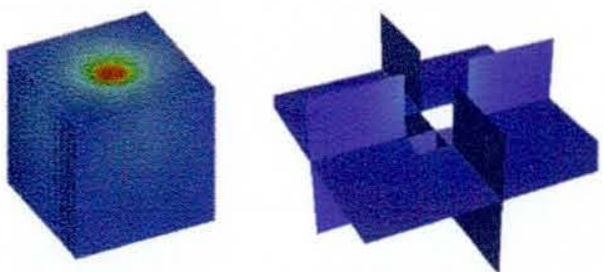

(a) $100 \mathrm{ps}$
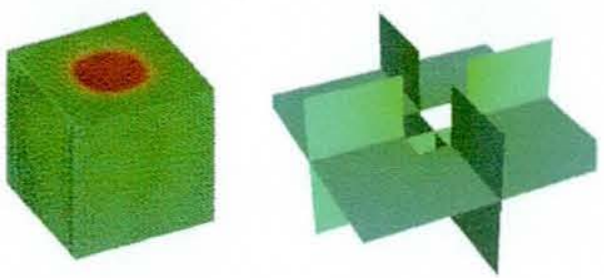
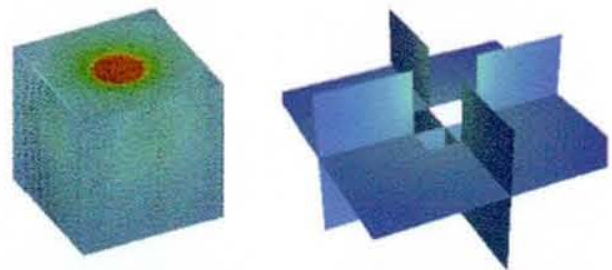

(b) $150 \mathrm{ps}$

(c) 200ps
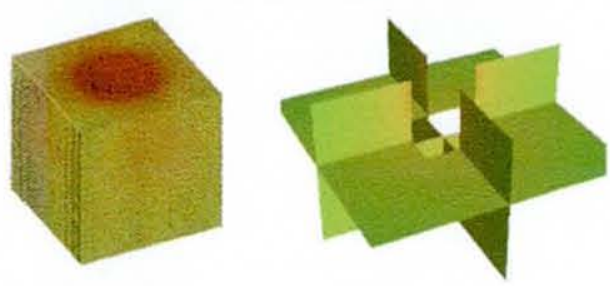

(d) $250 \mathrm{ps}$

Figure 6.5: Images from the 3D position matching model test simulation. The strain field spreads throughout the material in both the atomistic and continuum regions.

during the first 200 ps. The energy should then remain constant for the rest of the simulation as the indenter is fixed and no more work is done on the substrate, however the position matching method does not apply equal and opposite forces at the interface and as a result the total system energy is not conserved during this period and instead rapidly increases.

Further analysis of the interface in figure 6.7 shows that the face atoms on the boundary of the transition region experience increasing large vibrations as the energy is artificially added to the system.

To attempt to control the energy increase in the coupled model, energy needs to be removed from system to balance that being added. The Berendsen thermostat (see section 3.2.3) was applied to free atoms near the transition region to damp out kinetic energy. Figure 6.8(a) shows the total energy of the undamped system compared to the damped system during the $400 \mathrm{ps}$ 


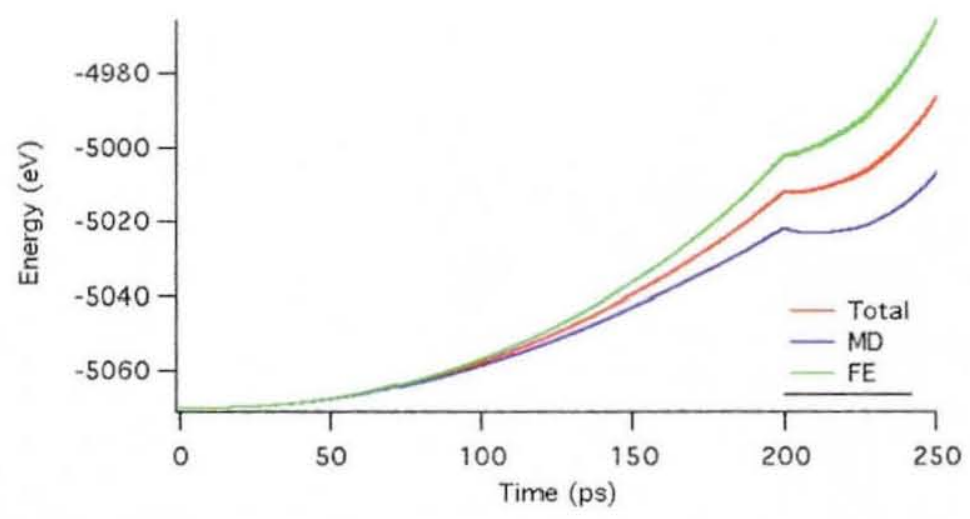

Figure 6.6: Energy in the atomistic and continuum regions and the total energy of the $3 \mathrm{D}$ position matching method during the simulation.

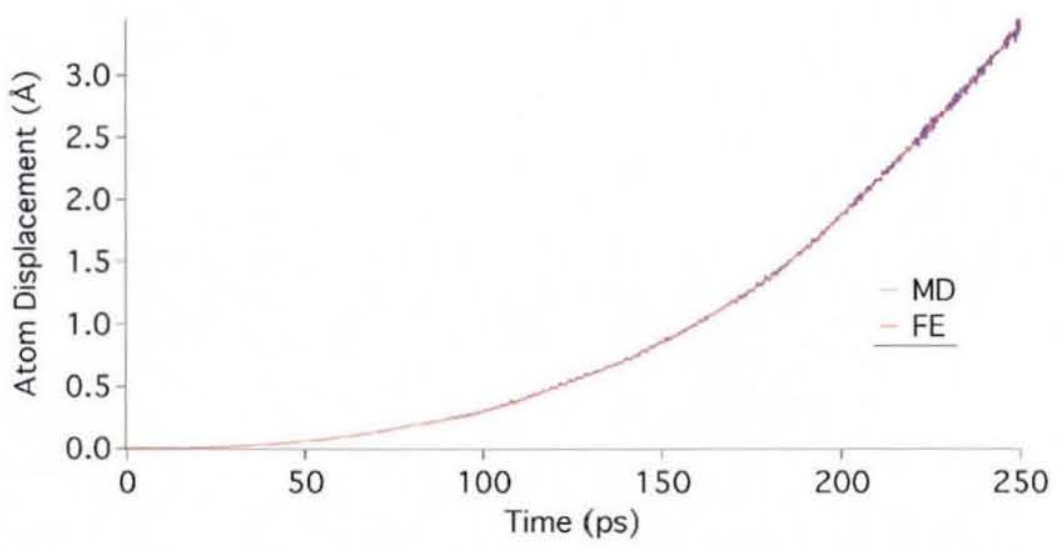

Figure 6.7: The comparison of the displacement of a face atom in the transition region. Its actual position computed by MD is shown in blue and the interpolated position due to the FE model is shown in red.

simulation. Although the damping controls the increase in energy and delays the initial rapid increase from $40 \mathrm{ps}$ in the undamped case to $60 \mathrm{ps}$ in the damped case, figure 6.8 (b) shows that the increase in energy in the damped simulation is still over $300 \mathrm{eV}$ in $400 \mathrm{ps}$. 


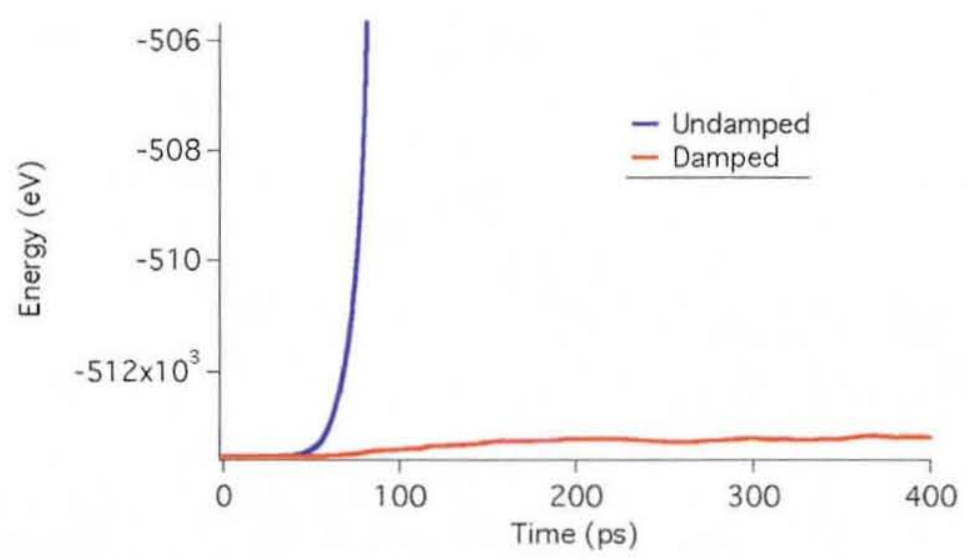

(a)

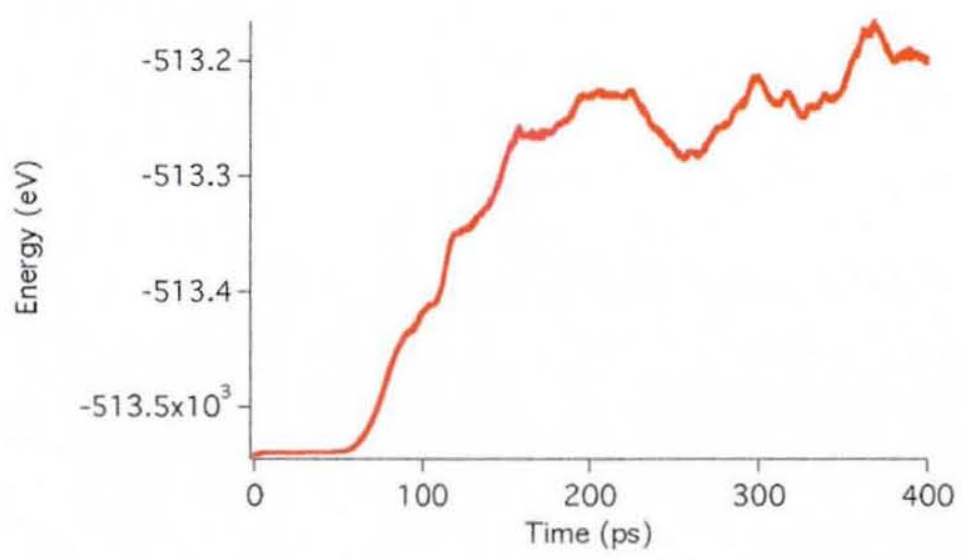

(b)

Figure 6.8: The effect of damping the atoms near the interface to remove the energy increase. (a) shows the energy in the damped and undamped cases. (b) shows only the damped case.

\subsubsection{Conclusions}

The results from the indentation simulation show that the position matching coupling technique allows interaction between an atomistic region and an embedding continuum region. This is achieved through the transition region which resolves the non-local to local mismatch of description of parti- 
cle interactions. The long range strain field due to the nanoindentation was successfully transmitted from the atomistic region into the far field modelled by the FE model. However, the position matching method does not apply equal and opposite forces in and around the transition region between the atomistic and continuum regions, and therefore does not conserve energy.

The nature of a coupled model is that there is constant feedback between the various descriptions of the material. This feedback amplifies the energy error at each time step leading to an exponential growth, and although the application of the Berendsen thermostat to remove energy near the transition region did reduce the energy increase, it was not sufficient to allow the method to model accurately any real nanoindentation experiment.

\subsection{Force Attribution Coupled Model}

The development of the method described here was driven by the constraint that Newton's third law of motion must be strictly obeyed. In doing so, the energy conservation of the coupled system is guaranteed to allow the method to be applied to simulations taking place over many thousands of time steps. Unlike the position matching technique, where particles have their forces described exclusively by atomistic or continuum mechanics, the force attribution method allows particles of the continuum region to be described by a combination of atomistic and continuum interactions. This allows the transformation from a non-local description of the atomistic region to a local description of the continuum region while still obeying Newton's third law. 


\subsubsection{Methodology}

The atomistic and continuum regions are linked together through an overlap region at the interface. It is this overlap that allows the two models to interact with each other. Figure 6.9 shows a cross-section of the atomistic region with a slice through the mesh of the continuum region in the same plane as that from which the cross section is taken. To describe the atomistics at the interface region three types of atom are defined; free atoms, imaginary atoms and node-atoms. The main atomistic region is made up of free atoms which are modelled in the usual way by MD. Around this bulk of free atoms is a regular grid of node-atoms (corresponding to the nodes of the FE mesh), and a skin of imaginary atoms which will be used to pass information from the atomistic region to the continuum region and vice-versa. In this case the node-atoms match the positions of the boundary nodes of the FE mesh, however, this is not a strict requirement of the force attribution method. This is a major advantage of this method over the existing methods, as matching atom positions to node positions is not a trivial exercise for more complicated crystal structures.

Imaginary atoms around the outside of the atomistic region make up the neighbours of free atoms near the interface. Therefore, the number of layers of imaginary atoms in the overlap of the two regions is dependent on the potential function being used, such that all free atoms have neighbours up to the potential's full cut-off distance. The size of the elements on the boundary is made large enough so that all of the imaginary atoms are within the first layer of elements, and the positions of the nodes of these elements that lie on

the boundary of the continuum region are made to coincide with node-atom positions. The node density then decreases with distance from the atomistic region, where larger elements represent larger numbers of atoms. 


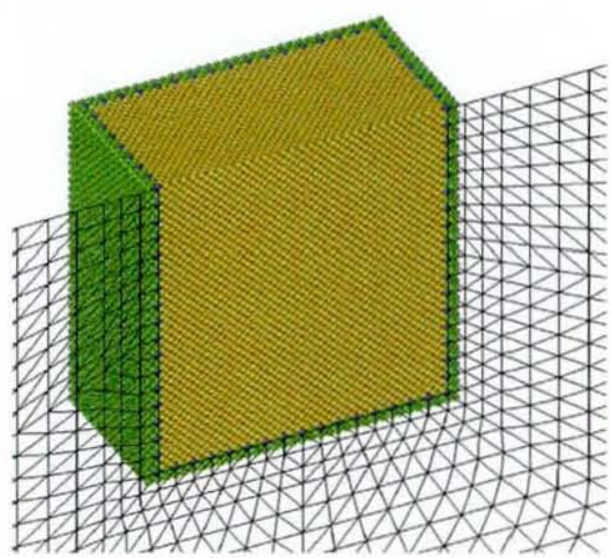

(a)

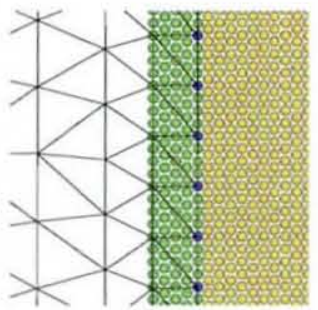

(b)

Figure 6.9: (a) Cross-section of the 3D coupling interface: The dark blue atoms near the edge of the atomistic region are node-atoms and coincide with FE nodes. The lighter layer of green atoms on the outside of the atomistic region are imaginary atoms. These lie within the first layer of elements in the continuum region. (b) Close-up of the arrangement at the interface.

The force calculation for all free atoms in the coupled model must be consistent. Free atoms near to the atomistic boundary include the forces due to imaginary atoms and node-atoms within the interface region to complete their force calculation. A one-dimensional representation of this is shown in figure 6.10. The second nearest neighbour interactions for free atoms that interact with imaginary atoms or node-atoms are shown, and due to Newton's third law, the equal and opposites of those forces are applied to the imaginary atoms.

To illustrate the procedure, consider node-atom 5 and imaginary atom 6 at the boundary as shown in figure 6.10. Atom 5 has a first nearest neighbour force from atom 4 and a second nearest neighbour force from atom 3, and 
imaginary atom 6 has a second nearest neighbour force from atom 4 . It is these forces that drive the interaction from the atomistic region to the continuum region, by apportioning the force to the nodes of the element in which that atom lies. The node-atom, labelled 5 in figure 6.10 , applies its whole force to the node with which it corresponds. The imaginary atoms use the linear shape functions of the element, $N_{i}$ to apportion their force. Thus atom 6 applies two thirds of its force to node 5 and a third to node 8 .

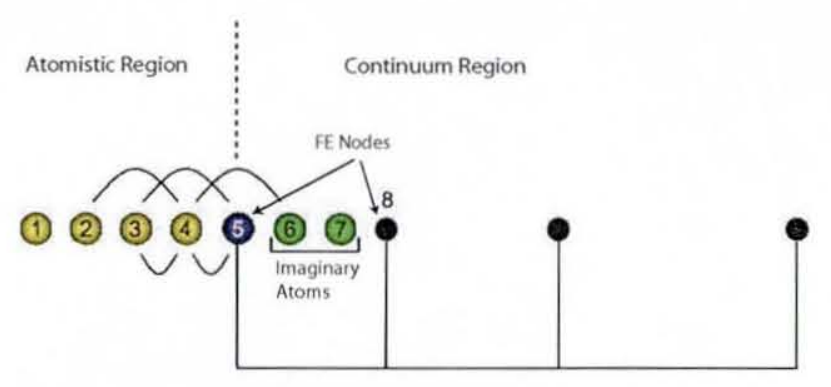

Figure 6.10: A 1-D representation of MD interactions at the interface: Atoms 1-4 are free atoms, modelled by MD. Node-atom 5 coincides with the first node of the FE region and atoms 6 and 7 are imaginary atoms within the first element in the FE region. First and second nearest neighbour interactions near the interface are shown.

For a three-dimensional tetrahedral element at the interface, containing $n_{a}$ atoms, the forces on its nodes are calculated by

$$
F_{\text {nde }}=\left.\sum_{i=1}^{n_{a}} N_{\text {nde }}\right|_{i} F_{i}
$$

where $F_{n d e}$ is the force on node $n d e,\left.N_{n d e}\right|_{i}$ is the value of the shape function of node nde at the position of atom $i$, and $F_{i}$ is the force on atom $i$. These forces are added to the usual nodal forces due to nodal displacements from the FE model and the positions are updated. 
According to the method described, the motion of the free atoms in the atomistic region causes the distortion of material in the continuum region. To complete the coupling of the two models, once the continuum region has undergone a distortion, interaction must also take place from the continuum region to the atomistic region. The same linear interpolation procedure as described for the position matching method in section 6.4 is used to update the position of the imaginary atoms in this method.

\subsubsection{Testing and Results}

A nanoindentation simulation into a lattice with first nearest neighbour Lennard-Jones interactions has been performed to test the force attribution method. This simulation is to investigate the passing of the strain field produced by the indentation, from the atomistic region into the continuum region, and also to check that the combined energy in the system is conserved after the indentation has occurred with the indenter held fixed for a lengthy period. The dimensions of the atomistic and continuum regions and the number of atoms and nodes in the respective regions are given in table 6.3. The total atoms in this table gives an approximate value of the number of atoms represented by the combined atomistic and continuum regions. The coupled model is in the region of 60 times faster than if this many atoms had been modelled by molecular dynamics alone.

A hard spherical indenter of radius $30 \AA$ has been used to indent into the fcc lattice modelled by the Lennard-Jones potential. As a first approximation to describe the interaction between the tip and the atoms in the substrate, the repulsive part of a Lennard-Jones potential has been used, as given in equation 6.11, where $r$ is the distance from the spherical tip surface to the atom, $\epsilon=7.8 \times 10^{-4} \mathrm{eV}$ and $\sigma=1.08 \AA$. This gives a large repulsive force 


\begin{tabular}{c|c}
\hline Parameter & Value \\
\hline \hline MD dimensions $\left(\AA^{3}\right)$ & $139 \times 139 \times 143$ \\
FE dimensions $\left(\AA^{3}\right)$ & $748 \times 748 \times 397$ \\
MD atoms & 169,016 \\
FE nodes & 32,860 \\
Total atoms & $\approx 13.1 \times 10^{6}$
\end{tabular}

Table 6.3: The system size for the force attribution method test simulation. Total atoms is the approximate number of atoms represented by the combined atomistic and continuum regions.

as the tip gets close to the substrate, but drops off quickly as it gets further away.

$$
F_{L J}(r)=48 \epsilon \frac{\sigma^{12}}{r^{13}}
$$

The lowest point of the indenter starts $2 \AA$ above the surface and is moved vertically downwards a distance of $3 \AA$ over a period of 60 ps where it is held for a further $40 \mathrm{ps}$. The resulting strain field spreads from the point of indentation towards the interface of the two models. The initial temperature is assumed to be $0 \mathrm{~K}$, with no heat bath to control temperature fluctuations. As a result there is a small rise in temperature of the system during the indentation phase.

Images of the strain field crossing the interface are shown in figure 6.11, where snapshots from the simulation are taken at 20ps intervals from 40ps to 100ps. The atomistic region, magnified and displayed on the left hand side, is taken from the void in the continuum region on the right hand side. To be able to visualise what is happening in the continuum region, orthogonal 
plates are drawn at the centre of the material in the $x$ and $y$ directions, and at the bottom of the atomistic region in the $z$ direction.

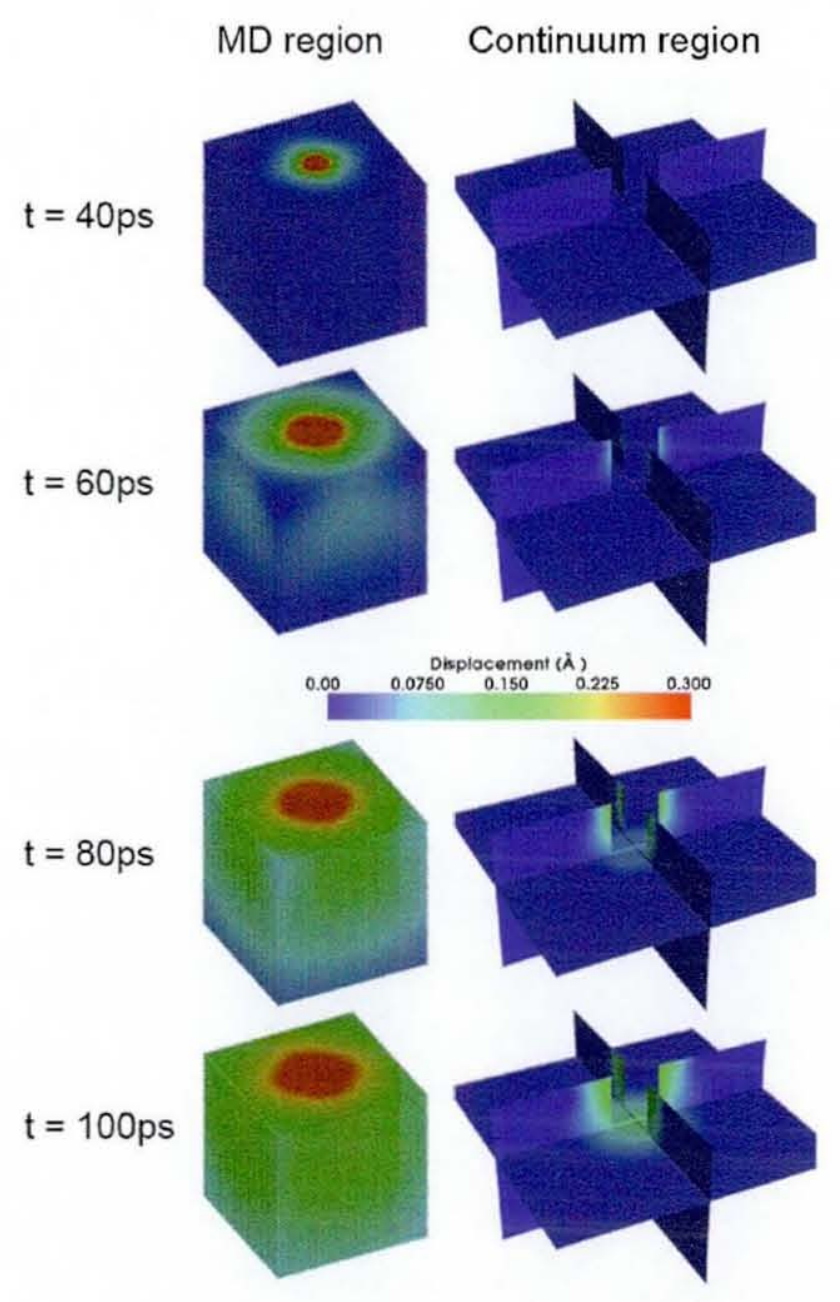

Figure 6.11: Images of how the strain field due to indentation spreads through the coupled interface. Snap shots are taken at 40ps, 60ps, $80 \mathrm{ps}$ and 100ps.

At 40ps the strain field is still localised around the point of indentation, there are relatively large displacements where the tip is interacting with the substrate, but the displacements are so small at the interface that they do not show up on the scale given in figure 6.11. By 60ps the strain field 
has spread into the continuum region. The displacements are even larger at the indentation as the tip is still being moved into the material and the displacements at the interface can clearly be seen with values of around $0.07 \AA$. At 80 ps displacements at the point of indentation have not changed much due to the holding of the tip, however the strain field has carried on moving much further into the continuum region where displacements can be seen up to as much as $0.2 \AA$. Finally at 100 ps the tip is still held in position and again there is little change around this point, but now the strain field has propagated far into the continuum region.

The total energy of the system is calculated by adding the potential and kinetic energies of the particles updated due to the MD model, and the potential and kinetic energies of the nodes in the continuum region. Figure 6.12 shows the energy for the two regions and the total energy of the coupled system during the simulation.

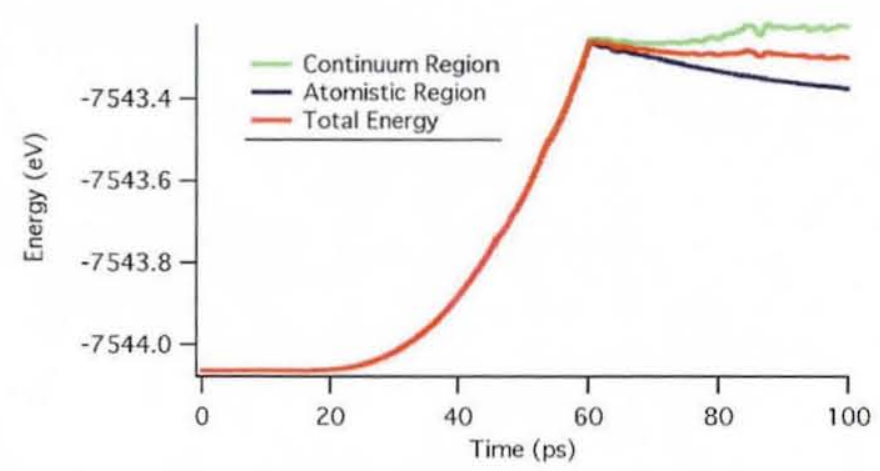

Figure 6.12: Energy in the atomistic and continuum regions and the total energy of the coupled system during the simulation. The energy in the continuum region is shown relative to the total energy.

The total energy increases during the indentation period as expected since work is done on the system. For the last 40ps of the simulation the indenter 
is held fixed. The continued propagation of the strain field causes an increase in the energy in the continuum region, however this is mirrored by a decrease in energy in the atomistic region giving energy conservation for the coupled system. This implies that the coupling strategy at the interface is correct.

During indentation there is an increase in kinetic energy due to the motion of particles which causes an increase in temperature of the substrate. In reality, this kinetic energy would propagate into the far field and only a little heating would occur at the point of indentation. However if a simulation is performed with fixed boundary conditions, this energy is reflected back into the system. Figure 6.13 compares the energy change between the two methods.

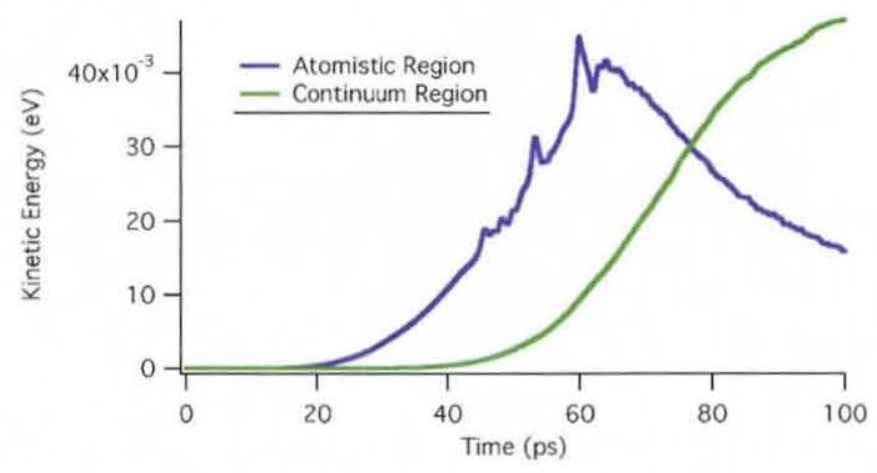

Figure 6.13: Kinetic energy in the atomistic and continuum regions. The energy is able to pass from the atomistic region into the continuum region to prevent artificial heating of the atoms.

This illustrates the necessity of coupling edge atoms to a heat bath to extract excess energy when running purely atomistic simulations on small systems. 


\subsubsection{Conclusions}

The energy conservation results and the smooth passage of waves between regions from the force attribution coupling technique test simulation are a good indication that the method is correct. The strain field due to the small indent passes smoothly from the atomistic region into the continuum region as required. The method strictly obeys Newton's third law of motion, and as a result the total system energy is conserved during the 40 ps holding period after the indentation has occurred. The local heating seen in atomistic simulations of nanoindentation is removed by the coupled model. The kinetic energy is able to pass into the far field and is not reflected back into the atomistic region. This provides a more realistic method of energy diffusion than to damp the boundaries of the atomistic region in order to control the lattice temperature.

The non-local/local mismatch at the interface gives rise to an imbalance of forces at regular lattice sites, the same as the ghost forces seen in the QC method (see section 4.3). However, in this method all atoms in the atomistic region are treated correctly as atoms in an MD only simulation. Hence, no correction needs to be done in the atomistic region. The imbalance can also be resolved by applying the ghost force correction scheme of the QC method at each time step, but in this case only to the linear elastic finite element region, or by simply relaxing the continuum region at the beginning of the simulation.

The force attribution coupling method will now be used in more realistic simulations in chapter 7, where the Ackland potential is applied in the atomistic region to model nanoindentation and laser ablation experiments. 


\section{Chapter 7}

\section{Applications of Multiscale Modelling}

The force attribution method is applied to simulate nanoindentation of $\mathrm{Au}$ and Fe and laser ablation of Au. The Ackland potential, described in section 2.2.2, is used to describe the interatomic interactions in the atomistic region, with the corresponding matched elastic constants calculated in section 6.2 applied to the FE model in the continuum region.

\subsection{Nanoindentation}

\subsubsection{Au $15 \AA$ Indentation}

A $15 \AA$ indentation into Au has been simulated using both the coupled model and an atomistic only simulation with fixed boundary conditions to investigate the effects of modelling linear elastic deformation in the far field. Table 7.1 gives the dimensions of the lattice used in the simulation. The same indentation model as used in section 6.5.2 is used here, describing the indenter- 
substrate interactions by the repulsive part of the Lennard-Jones potential function. A $15 \AA$ tip displacement at constant velocity takes place over the first $300 \mathrm{ps}$ of the simulation. The indenter is then removed over the next 100 ps so that it has been totally removed from the material. Comparisons will be made between the two simulations, first for the contact pressure at the maximum load, secondly for the differences in deformation of the material, and finally for the temperature of the atomistic region.

\begin{tabular}{c|c}
\hline Parameter & Value \\
\hline \hline MD dimensions $\left(\AA^{3}\right)$ & $147 \times 147 \times 147$ \\
FE dimensions $\left(\AA^{3}\right)$ & $748 \times 748 \times 397$ \\
MD atoms & 194,509 \\
FE nodes & 32,860 \\
Total atoms & $\approx 13.1 \times 10^{6}$
\end{tabular}

Table 7.1: The coupled system size used for the Au indentation simulation.

Figure 7.1(a) shows a comparison of the normal force on the indenter as a function of indentation depth. The atomistic only model with fixed boundary conditions shows the first pop-in (which occur due to dislocation emission [3]) at about $6 \AA$ contact depth, and a maximum load of $280 \mathrm{nN}$ at $15 \AA$. In comparison, the coupled model of a much larger system takes longer to produce any plastic deformation, with the first pop-in at around 9 $\AA$ contact depth but with the same applied force. The peak load at $15 \AA$ is $155 \mathrm{nN}$, far lower than in the atomistic only simulation. The graph of total system energy is shown in figure 7.1(b). It is clear that more work has been done in the atomistic only simulation to achieve the same displacement of the indenter.

The contact pressure is defined as the ratio of the normal load to the 


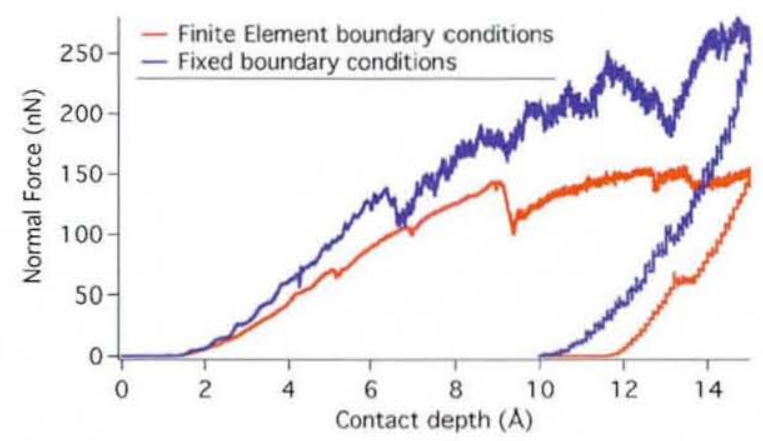

(a)

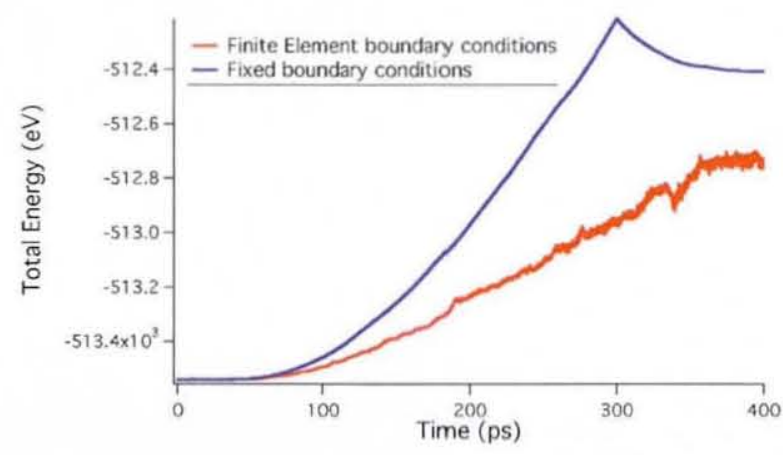

(b)

Figure 7.1: (a) The normal force on the indenter versus indentation depth for both the coupled method and the atomistic only simulations. Note that the first pop-in occurs at the same normal force of $\approx 125 \mathrm{nN}$. (b) The change in total system energy during the simulations.

contact area during indentation and will be used to compare the hardness of the material in the two simulations. These have been calculated from figure 7.1(a) and from a measure of the cross-sectional area of the hole created at maximum indentation depth from the computer image of the surface. The results for $\mathrm{Au}$ are displayed in table 7.2 along with results for other metals calculated using MD alone in [49]. The elastic compression of the substrate in the continuum region affects the total displacement of the atomistic region. 
The entire atomistic region moves down $10 \AA$ at the maximum indentation depth. This compression results in a far smaller hole depth for the same indenter displacement, and due to the depth dependency at this scale, the contact pressure for this simulation should be compared to smaller indentation depths using atomistics only for a fair comparison. To obtain a similar hole depth to that in the atomistic only simulation an indenter displacement of more than double the size is required. The result from this simulation shows that embedding the atomistic region in a continuum region almost halves the contact pressure at the maximum depth. Clearly these depths are much smaller than those in experimental work, but the indentation size effect $[50,51]$ for these metals is clearly demonstrated.

\begin{tabular}{|c|c|c|}
\hline Substrate & $\begin{array}{l}\text { Maximum } \\
\text { Hole } \\
\text { Depth }(\AA)\end{array}$ & $\begin{array}{l}\text { Contact } \\
\text { Pressure } \\
(\mathrm{GPa})\end{array}$ \\
\hline \multicolumn{3}{|c|}{$\begin{array}{c}\text { Indenter displacement of } 15 \AA \\
\text { Atomistics only }\end{array}$} \\
\hline $\mathrm{Fe}$ & 8.8 & 50.3 \\
\hline $\mathrm{Ag}$ & 10.7 & 24.1 \\
\hline $\mathrm{Au}$ & 13.8 & 19.6 \\
\hline \multicolumn{3}{|c|}{$\begin{array}{c}\text { Indenter displacement of } 31 \AA \\
\text { Coupled model }\end{array}$} \\
\hline $\mathrm{Au}$ & 14.5 & 10.4 \\
\hline \multicolumn{3}{|c|}{$\begin{array}{c}\text { Indenter displacement of } 15 \AA \\
\text { Coupled model }\end{array}$} \\
\hline $\mathrm{Au}$ & 4.8 & 17.6 \\
\hline \multicolumn{3}{|c|}{$\begin{array}{c}\text { Indenter displacement of } 5 \AA \\
\text { Atomistics only }\end{array}$} \\
\hline $\mathrm{Fe}$ & 1.0 & 144.5 \\
\hline $\mathrm{Ag}$ & 2.4 & 41.9 \\
\hline
\end{tabular}

Table 7.2: Effective indentation depths and contact pressures for different metals. Results for $\mathrm{Fe}$ and $\mathrm{Ag}$ are given for indenter displacements of 15 and $5 \AA$ to give comparative hole depths for the Au results. 
Previously for atomistic only simulations [49], it had been observed that for even larger indentation depths the values of the determined contact pressure were always very high. The results from the coupled model imply that this may be due to the neglect of the embedding elastic region in addition to the indentation size effect.

The elastic and plastic deformation of material can be seen for the two simulations at the maximum indentation depth and after the tip has been extracted in figure 7.2. The pile-up of atoms on the surface is clearly much higher in the simulation using atomistics only. Table 7.3 shows there are 10 times as many atoms piled-up than in the simulation with the coupled model, and also the elastic recovery of the hole with respect to the maximum hole depth is far greater with the coupled model.

\begin{tabular}{lcc}
\hline & Coupled model & Atomistics only \\
\hline Atoms pile-up (end) & 63 & 635 \\
Elastic recovery $(\AA \mid \%)$ & $2.4 \mid 50$ & $3.5 \mid 25.4$ \\
First dislocation emission (ps) & 104 & 88 \\
Slip atoms (max. depth) & 1693 & 4294 \\
Slip atoms (end) & 769 & 4027
\end{tabular}

Table 7.3: Parameters to investigate elastic and plastic deformation due to the indentation. Elastic recovery is given as both a distance and as a percentage of the maximum hole depth.

Dislocations in the material are caused by pressure created under the tip. Table 7.3 shows that it takes a longer time, and hence larger displacement of the indenter, to cause the first dislocation using the coupled model than with the atomistic only simulation. This occurs because of the slower increase in normal load. In figure 7.1(a), there is a small drop in the normal force at a depth of $4.4 \AA$ (which corresponds to 88 ps) in the curve for the atomistic only simulation. At a depth of $5.2 \AA$ (corresponding to $104 \mathrm{ps}$ ), there is a 


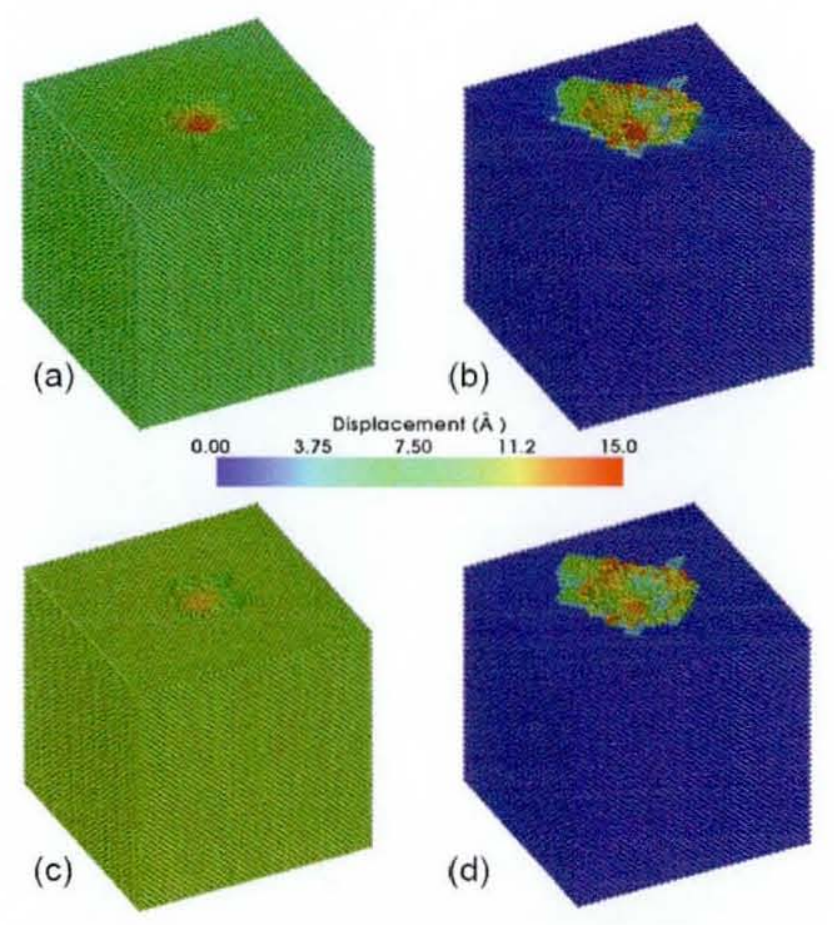

Figure 7.2: Images of damage due to indentation. (a) and (c) are from the coupled model at maximum indentation depth and after extraction respectively. (b) and (d) are from the atomistic only simulation, also at maximum indentation depth and after extraction respectively. Atoms are coloured according to how much they have been displaced from their original position.

drop in normal force due to the dislocation emission in the coupled model. These both occur at the same normal force. Then again at a depth of $6.3 \AA$ in the atomistic only simulation and $9 \AA$ in the coupled model there are large drops in the normal force, which is again the same value in both simulations.

The difference in the amount of dislocation emission in the two simulations is shown in figure 7.3, in the atomistic only simulation there are far more activated slip planes at the maximum indentation depth than in the coupled model. Plastic damage under the tip after extraction is also more 
pronounced in the atomistic only simulation, table 7.3 shows that there are more permanently displaced atoms.
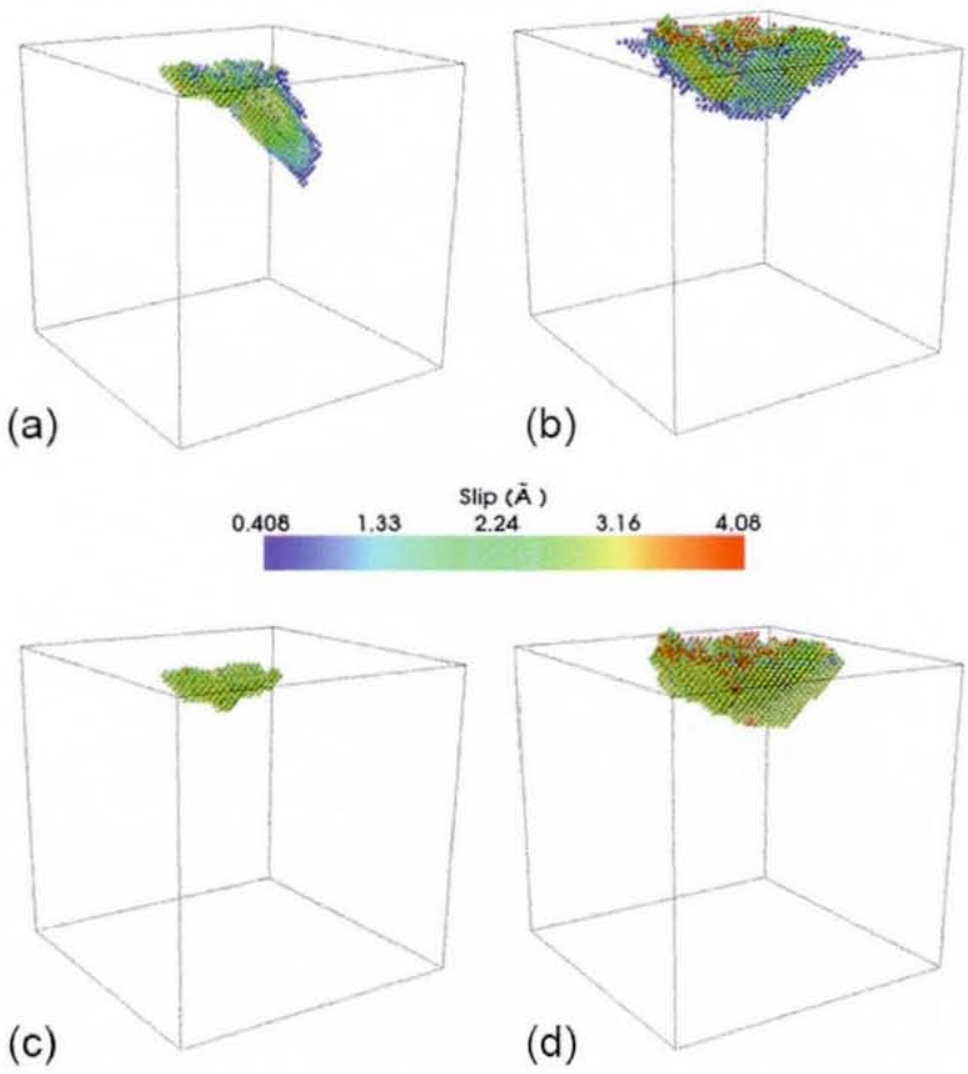

Figure 7.3: Images of slip underneath the indenter. (a) and (c) are from the coupled model at maximum indentation depth and after extraction respectively. (b) and (d) are from the atomistic only simulation, also at maximum indentation depth and after extraction respectively.

The presence of the FE surround also allows the energy induced during indentation to be more efficiently dispersed. This is shown in figure 7.4. The kinetic energy in the atomistic region at the end of the simulation is used to calculate the average temperature. For the simulation with fixed boundary 


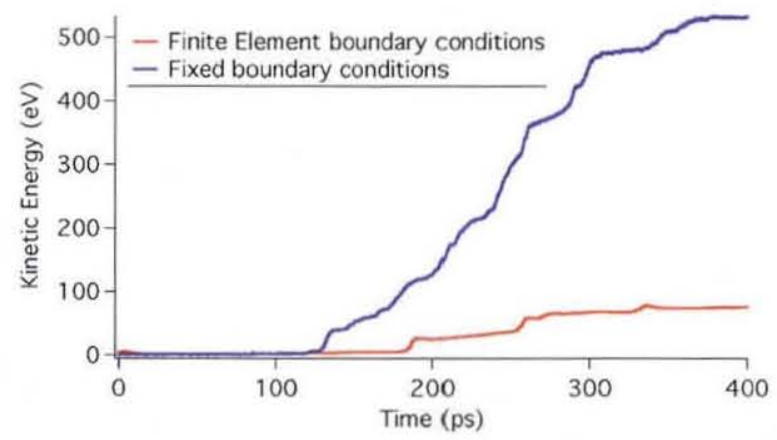

Figure 7.4: Graph showing the kinetic energy in the atomistic region during the simulation. Results are shown for both the coupled model and the atomistic only simulation.

conditions around the atomistic region, the final kinetic energy is $534 \mathrm{eV}$, giving a lattice temperature of $31 \mathrm{~K}$. The coupled model allows this energy to be passed into the continuum region, hence reducing the final kinetic energy in the atomistic region to $76 \mathrm{eV}$, a lattice temperature of $4.3 \mathrm{~K}$.

\subsubsection{Fe $15 \AA$ Indentation}

The indentation simulation carried out on the $\mathrm{Au}$ lattice in the previous section has been performed on a Fe substrate with the coupled model with the same $15 \AA$ indenter displacement and the same indentation speed. The dimensions of the substrate used in the simulation are given in table 7.4. Again, the same indentation model is used as described in section 6.5.2, where the indenter-substrate interactions are described by the repulsive part of the Lennard-Jones potential.

The force-depth curve in figure 7.5 shows that the substrate experiences elastic deformation up to a depth of around $10 \AA$. The continued indentation then produces plastic deformation of the substrate for a further $5 \AA$ to the 


\begin{tabular}{c|c}
\hline Parameter & Value \\
\hline \hline MD dimensions $\left(\AA^{3}\right)$ & $126 \times 126 \times 126$ \\
FE dimensions $\left(\AA^{3}\right)$ & $738 \times 738 \times 377$ \\
MD atoms & 176,309 \\
FE nodes & 71,141 \\
Total atoms & $\approx 18.1 \times 10^{6}$
\end{tabular}

Table 7.4: The coupled system size used for the Fe indentation simulation.

maximum indentation depth. The motion of the indenter is then reversed and is removed from the substrate after a further 150 ps when the indenter is still $6 \AA$ below its initial position, leaving a hole $5 \AA$ deep.

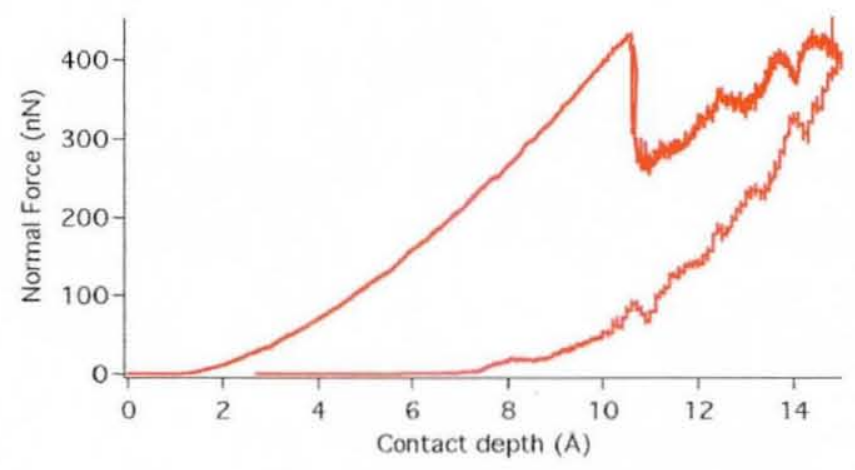

Figure 7.5: Force-depth curve for the $15 \AA$ indentation of Fe with the coupled model.

Images taken from the simulation during the elastic deformation period, at the maximum indentation depth and after the indenter has been removed, are shown in figure 7.6. After $150 \mathrm{ps}$, the indenter has created a hole depth of $6.5 \AA$ and the resulting strain field has started to propagate into the continuum region. At the maximum indentation depth, pile-up can be seen 
around the indentation and the strain field has increased in the continuum region. Displacements at the interface have reached around $1 \AA$. After the indenter has been removed, the pile-up around the hole remains. The hole depth elastically recovers by $9 \AA$, which is around $65 \%$ of the maximum hole depth. The indenter no longer applies stress to the material and hence the strain is removed from the continuum region, which elastically returns to its original shape.

The load at the maximum depth is shown in figure 7.5 to be $400 \mathrm{nN}$, and the hole diameter of $50.25 \AA$ at the maximum depth, gives a cross-sectional contact area of $A_{c}=1983.2 \AA^{2}$. The contact pressure is then calculated as $20.17 \mathrm{GPa}$ which is less than half of the value given in table 7.2 for the atomistic only simulation to the same depth. The result is still higher than the experimental value for $\mathrm{Fe}$ of $1.9 \mathrm{GPa}[2]$, however, this may be accounted for by the indentation size effect for this material.

\subsubsection{Conclusions}

The coupled model allows larger systems to be simulated while keeping the computation time reasonably low. Future work will enlarge system sizes even further to allow more realistic systems to be simulated. By embedding an atomistic region within a continuum region, the coupled model allows the long range strain field caused by indentation to cross the interface and be simulated in the far field. The compression of the continuum region causes a smaller hole to be created for the same indenter displacement and considerably less pile-up after extraction compared to atomistic only models with fixed boundary conditions. However, the dislocation features that occur are qualitatively the same in both models. The coupled model causes dislocation emission to take longer to occur and considerably reduces the plastic 

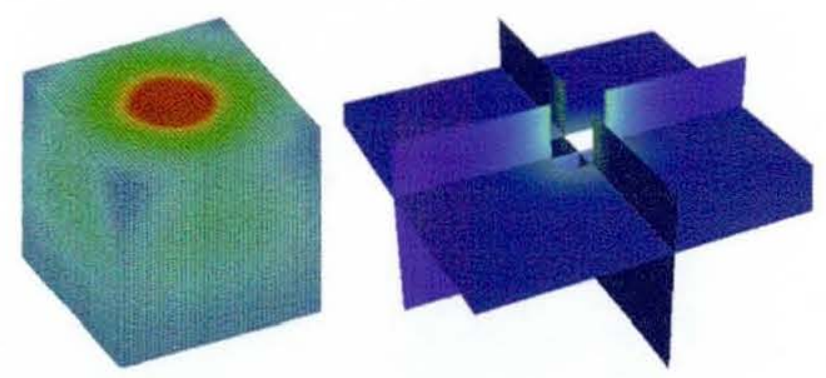

(a) $150 \mathrm{ps}$
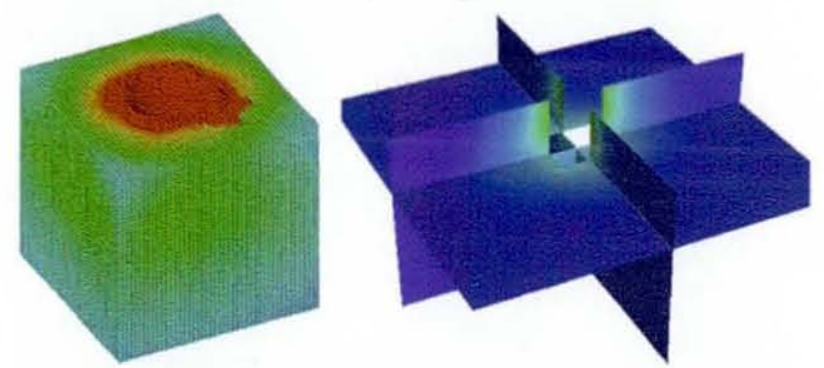

(b) $300 \mathrm{ps}$
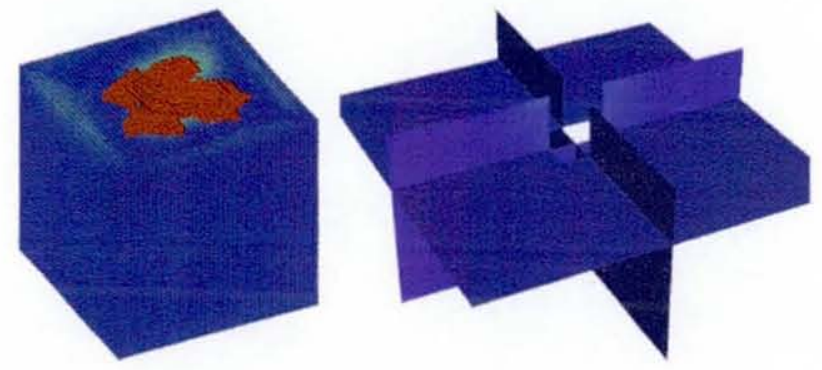

(c) $450 \mathrm{ps}$

Figure 7.6: Images from the $15 \AA$ Fe indentation: (a) during the elastic deformation period, (b) at the maximum indentation depth, and (c) after the indenter has been removed. The material is coloured on displacement from zero (dark blue) to $1.8 \AA$ and above (red).

damage under the tip after extraction. Using the coupled model, less work is done on the lattice to move the indenter the same distance than modelling atomistics only with fixed boundary conditions. This is due to the 
higher elastic distortion of the lattice in the FE region, and hence less plastic distortion in the MD region. The contact pressure value from the coupled model indicates that neglecting to model elastic compression in the far field may cause artificially high values for contact pressure in atomistic only simulations with fixed boundary conditions. The dislocation emission seen in nanoindentation experiments and atomistic only simulations travel quickly over large distances. In the coupled model, dislocations cannot pass through the transition region into the continuum region and plastic deformation is restricted to the atomistic region. A possible solution to this is discussed in chapter 8 .

\subsection{Laser Ablation}

Laser ablation is a process used to remove material from a solid by the application of light, in the form of a laser beam. Applications of laser ablation include, etching [52], drilling [53] and thin-film coatings [54, 55]. Energy from the laser beam is transfered to the atoms of the material causing some of them to be ejected from the substrate. The short femtosecond or nanosecond laser pulse of the beam applies the majority of the energy locally to atoms near where the laser hits the surface and hence heating of the surrounding material is minimal. However, heat waves are produced which travel at high speed into the far field away from the point where the laser beam is applied, making the experiment suitable for multiscale modelling.

\subsubsection{Au Laser Ablation}

The multiscale model has been applied to simulate laser ablation of Au with the same initial system setup as used in the indentation simulation in section 
7.1.1. As a first approximation to the experiment, the effect of the laser on the atoms in the substrate was modelled by applying initial velocities in random directions to 1065 atoms within $20 \AA$ radius hemisphere, the centre of which was at the centre of the atomistic region's free surface. The velocities were distributed normally within the hemisphere such that the maximum velocity of $0.018 \AA /$ fs was at the centre and reduced to $0.3 \%$ of the maximum at the outer edge, giving an average temperature of $7000 \mathrm{~K}$ and energy of around $1 \mathrm{keV}$ added to the system. The simulation then ran freely for $60 \mathrm{ps}$ for the heat wave to propagate into the far field. The identical initial conditions were applied to an atomistic only model to compare the results with those obtained from the coupled model in order to investigate the effects of the embedding continuum region.

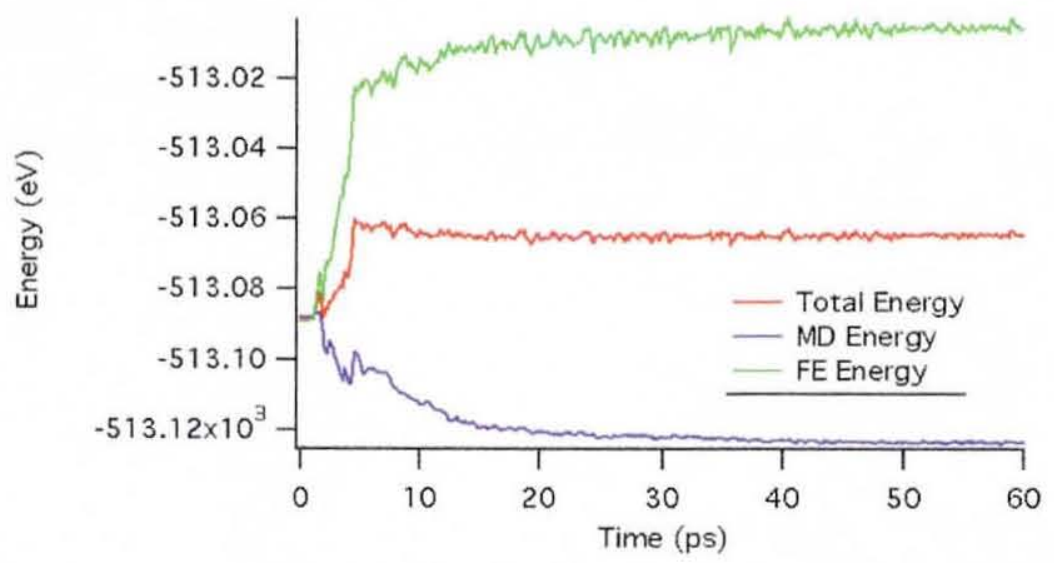

Figure 7.7: Graph showing the energy in the regions of the coupled model and the total system energy during the laser ablation simulation.

The energy in the atomistic and continuum regions and the combined system energy of the coupled model is shown in figure 7.7. This shows that the coupled model allows the majority of the energy due to the laser ablation to pass away from the atomistic region into the continuum region in the first 
$18 \mathrm{ps}$ of the simulation. Images of the simulation during this period are shown in figures 7.10 and 7.11 from the atomistic and continuum regions respectively. These images show the elastic and thermal waves generated in the atomistic region pass into the continuum region, while in the atomistic region, 10 atoms from the centre of the free surface are sputtered leaving a small hole.

Images of the atomistic only simulation are shown in figure 7.12 from the same times as the images from the coupled model. The elastic waves reach the edge of the lattice in the first $3 \mathrm{ps}$ of the simulation, but due to the fixed boundary conditions they are reflected back into the atomistic region. Hence, in figure 7.12 large distortions are still seen in the atomistic region compared to figure 7.10 from the same time in the coupled model. This continues throughout the simulation as the fixed boundary conditions restrict the energy from the laser ablation to the atomistic lattice. This is illustrated by figure 7.8 which shows the kinetic energy in the atomistic region is higher than in the coupled model where it is transfered into the continuum region. In this case there is no difference in the number of sputtered particles in the atomistic only and coupled simulations as the reflected waves do not cause any more atoms to be ejected, however this may not be the case in higher energy laser ablation simulations.

It is difficult to see the heat wave travelling through the continuum region from the images of the simulation due to the high velocities and displacements around the atomistic region. However, plotting the average particle temperature against distance from the laser application at regular time intervals illustrates the dissipation of the heat wave quite clearly in figure 7.9. This shows that the heat wave is moving away from the atomistic region at a speed of $1000 \mathrm{~m} / \mathrm{s}$, around half the speed of sound in $\mathrm{Au}$. 


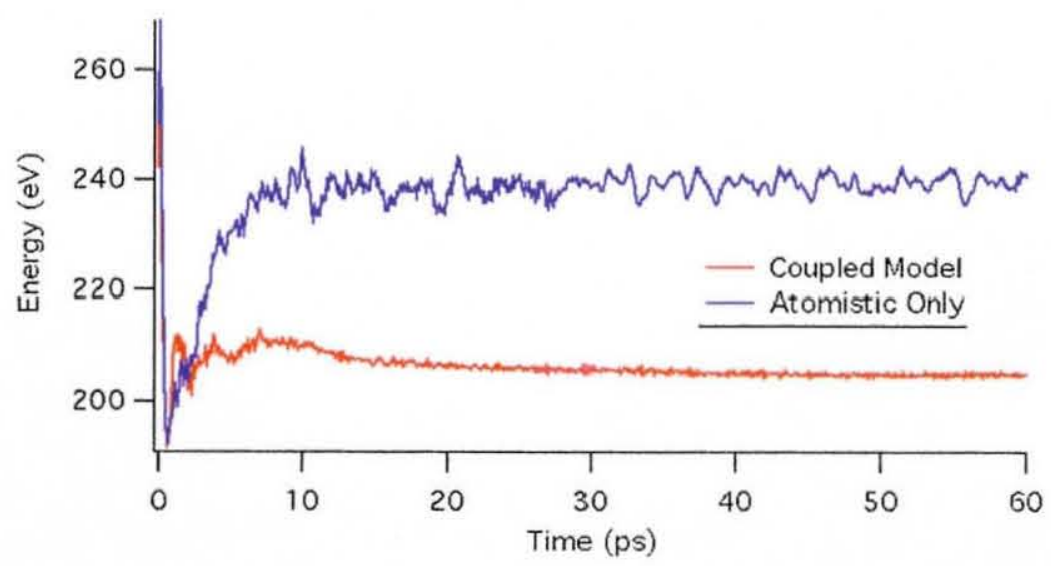

Figure 7.8: Graph showing the kinetic energy in the atomistic region for both the coupled and atomistic only models during the laser ablation simulation.

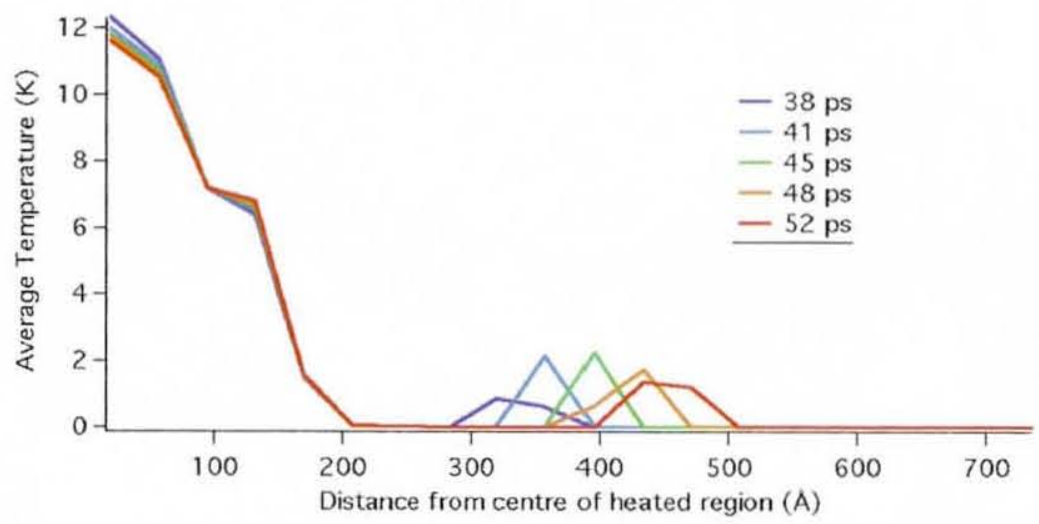

Figure 7.9: Graph showing the average particle temperature against distance from the laser application at various times during the laser ablation simulation. The heat wave is seen to travel through the continuum region away from the atomistic region.

\subsubsection{Conclusions}

The coupling of MD to FE enables the computing power in the coupled model to be concentrated on the region where plastic deformation occurs while in 


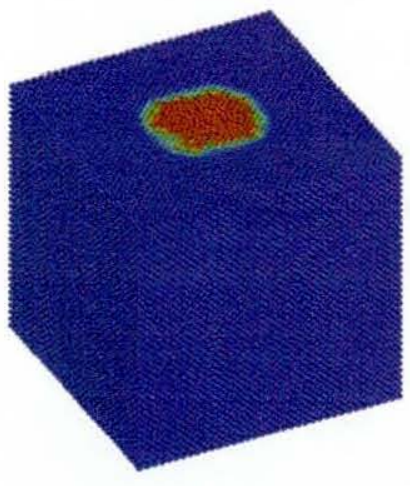

(a) $0.5 \mathrm{ps}$

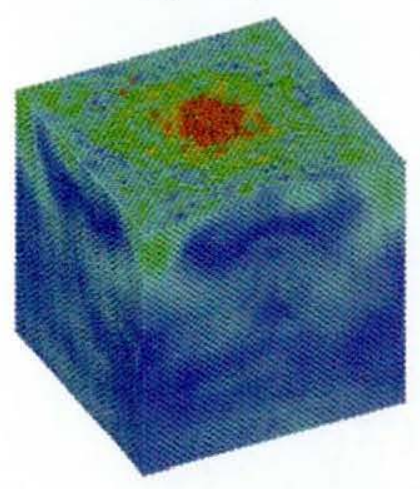

(d) $8.0 \mathrm{ps}$

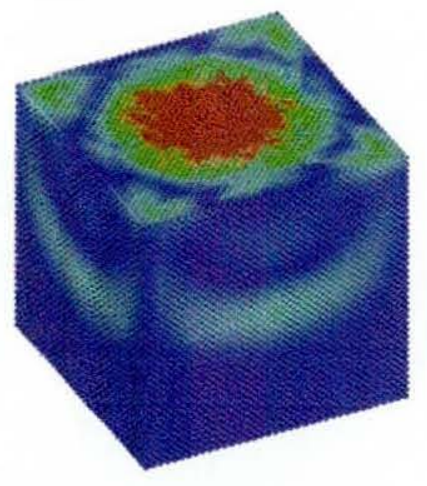

(b) $3.0 \mathrm{ps}$

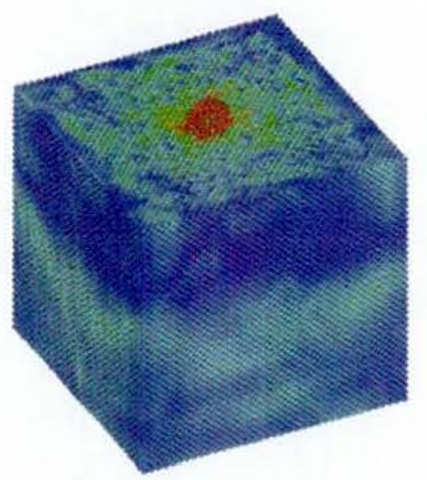

(e) $10.5 \mathrm{ps}$

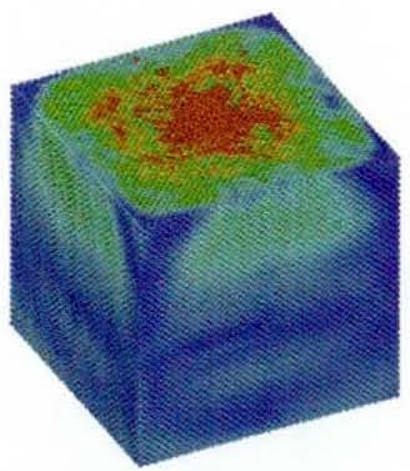

(c) $5.5 \mathrm{ps}$

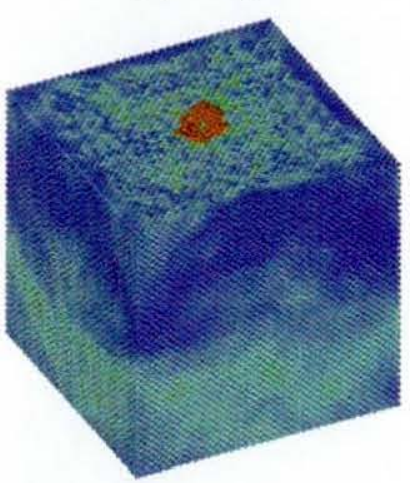

(f) $13.0 \mathrm{ps}$

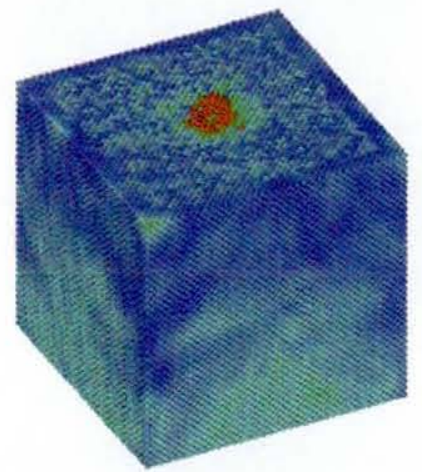

(g) $15.5 \mathrm{ps}$

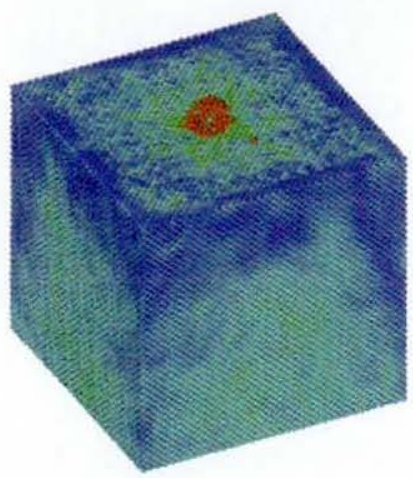

(h) $18.0 \mathrm{ps}$

Figure 7.10: Images of the atomistic region of the coupled model during the laser ablation simulation. 


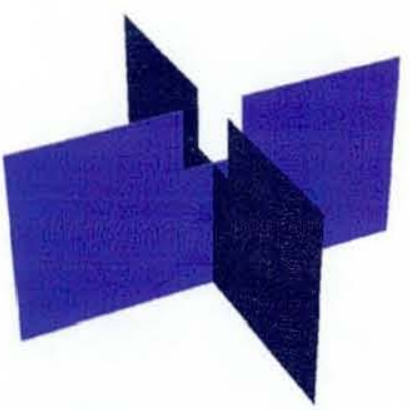

(a) $0.5 \mathrm{ps}$

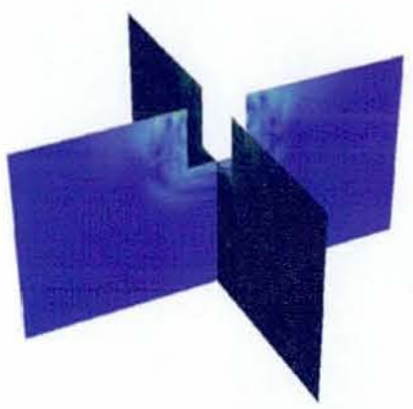

(d) $8.0 \mathrm{ps}$

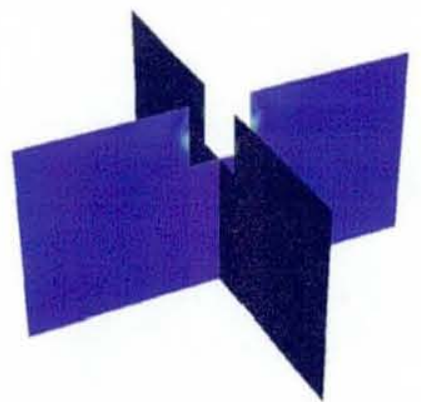

(b) $3.0 \mathrm{ps}$

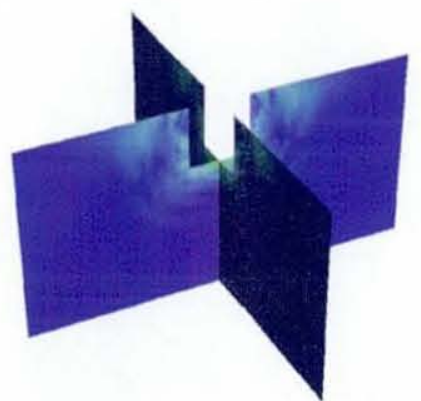

(e) $10.5 \mathrm{ps}$

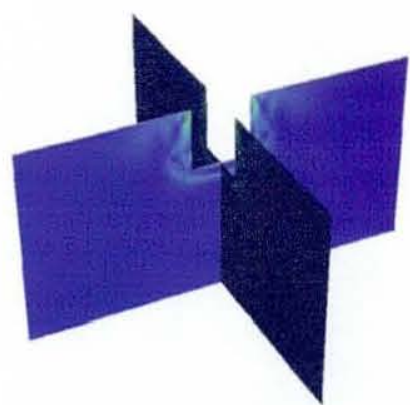

(c) $5.5 \mathrm{ps}$

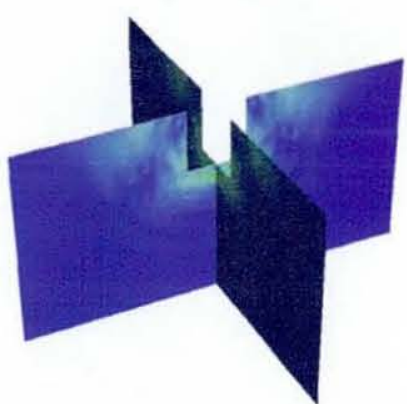

(f) $13.0 \mathrm{ps}$

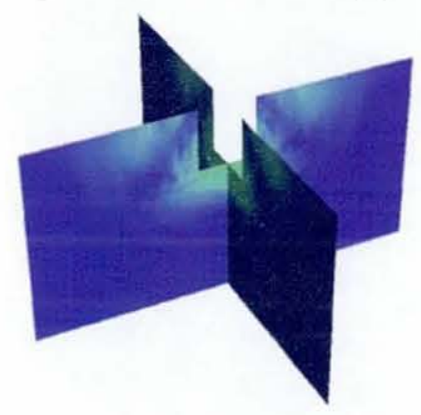

(g) $15.5 \mathrm{ps}$

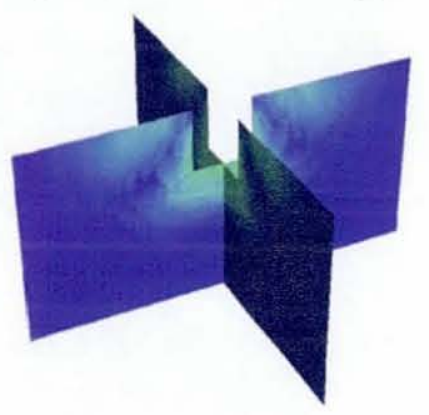

(h) $18.0 \mathrm{ps}$

Figure 7.11: Images of the continuum region of the coupled model during the laser ablation simulation. 


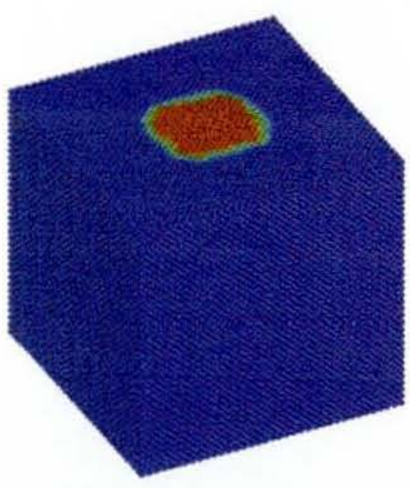

(a) $0.5 \mathrm{ps}$

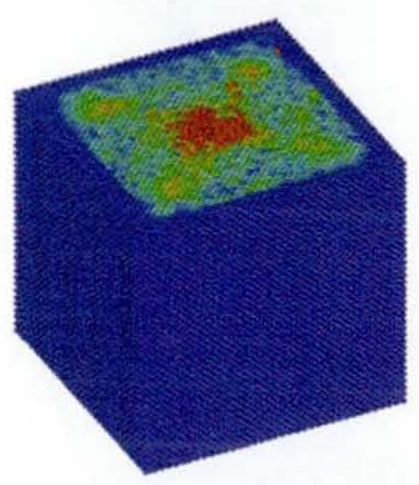

(d) $8.0 \mathrm{ps}$

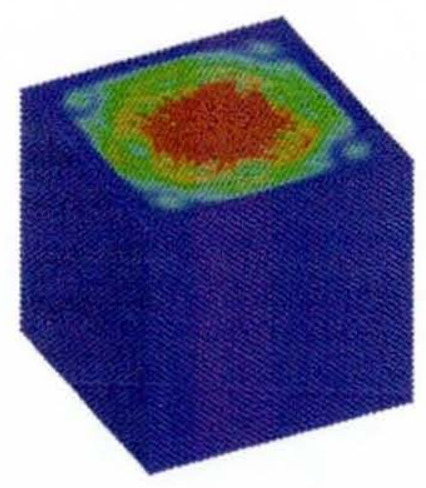

(b) $3.0 \mathrm{ps}$

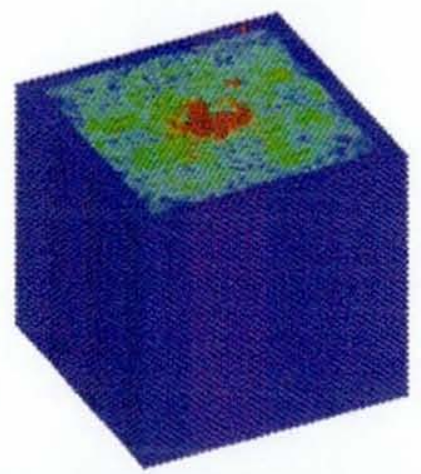

(e) $10.5 \mathrm{ps}$

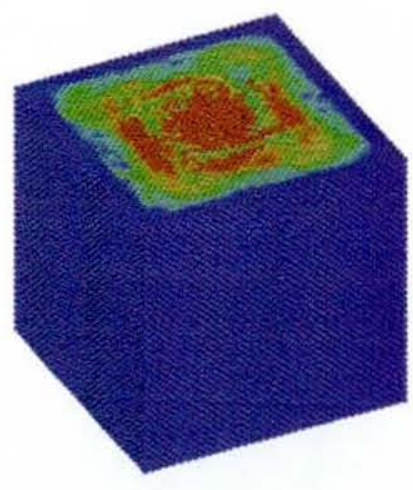

(c) $5.5 \mathrm{ps}$

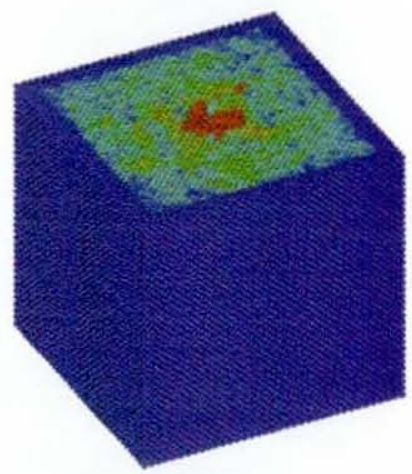

(f) $13.0 \mathrm{ps}$

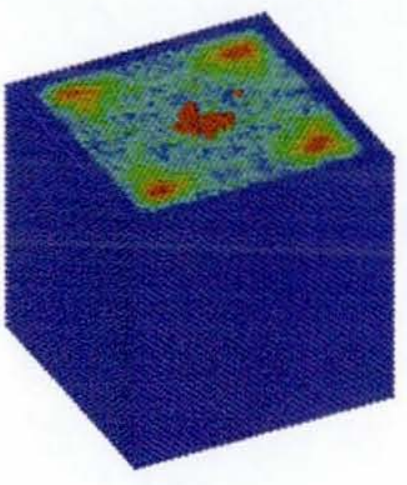

(g) $15.5 \mathrm{ps}$

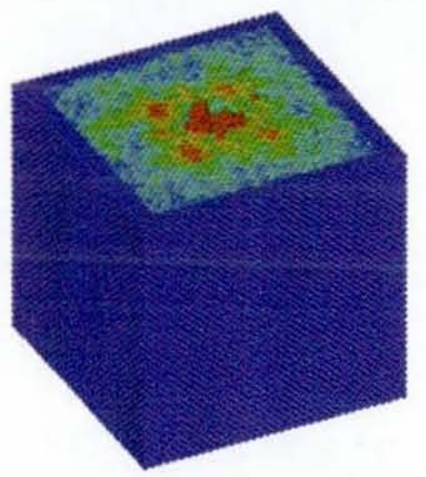

(h) $18.0 \mathrm{ps}$

Figure 7.12: Images of the atomistic only simulation during the laser ablation simulation. 
the far field a less computationally expensive solution is sufficient, allowing much larger systems to be modelled than by atomistic only models.

The heat wave generated by the laser ablation is able to pass from the atomistic region to be modelled in the far field by continuum mechanics while still modelling the material where the laser is applied by atomistics. This enables the kinetic energy from the laser ablation to be dissipated into the far field instead of being artificially constrained to the atomistic region. 


\section{Chapter 8}

\section{Conclusions and Future Work}

The results from atomistic simulations of nanoindentation, such as those in chapter 3 vary in their agreement with experimental results. For super hard substrates, such as diamond, the contact pressure and Young's modulus are very close to those obtained experimentally, whereas for softer materials, results for contact pressure and Young's modulus there is disagreement with experimental results, as seen in the silicon simulation. This may be due to inaccuracy in the interatomic potential function, for example, it has been observed that the Tersoff potential (section 2.2.3) does not match the elastic constants calculated from the experiment. Furthermore, the substrate lattices used in simulations have a perfect crystal lattice because the system sizes used are too small to model correctly the defect density seen in reality. The indentation depths in the simulations are much smaller than those in the experiment and it is known that the indentation size effect does cause a larger contact pressure value at these shallow depths. However, previous atomistic nanoindentation simulations have never been close to the experimental contact pressure value, even at much larger depths. It is suggested that the fixed boundary conditions applied in these models causes an over approximation 
to the substrates hardness due to the neglect of elastic compression in the far field.

In this work, multiscale modelling has been used to link an atomistic model to an embedding continuum model in order to remove the fixed boundary conditions from the atomistic model in nanoindentation simulations and provide the capability to model much larger systems than atomistic only models. The previous multiscale models discussed in chapter 4 provide a range of coupling schemes to link molecular dynamics to finite elements in order to bridge the length scales in materials modelling. However, each of the models discussed has disadvantages when considering its application to nanoindentation and hence a new method has been developed for this purpose.

The initial $2 \mathrm{D}$ model described in section 5.4 has a simple but accurate coupling of first and second nearest neighbour interactions. No interactions cross the boundary between the atomistic and continuum regions, therefore, so long as the elastic constants are matched correctly and only linear elastic deformation reaches the continuum region, the $2 \mathrm{D}$ model is able to couple seamlessly the MD and FE models. However, the simplicity of the model is also its downfall. No interactions may cross the boundary between the regions, and hence the model is restricted to specific cases. If extended to $3 \mathrm{D}$, only first nearest neighbour interactions could be considered in the body centred cubic and face centred cubic lattice structures, and hence the method has not been developed any further.

The position matching technique, initially developed in $2 \mathrm{D}$ and then extended into $3 \mathrm{D}$ is a generic model in the sense that any potential function with a finite cut-off distance may be applied in the atomistic region. The method allows interaction between an atomistic region and an embedding 
continuum region through a transition region which resolves the non-local to local mismatch of description of particle interactions. The testing of the method in $2 \mathrm{D}$ and $3 \mathrm{D}$ showed that long range strain fields due to nanoindentation are successfully transmitted from the atomistic region into the far field modelled by the FE model. However, the position matching method does not apply equal and opposite forces on atoms in and around the transition region, and therefore does not conserve energy.

The constant feedback between the various descriptions of the material amplifies the energy error at each time step leading to an exponential growth, and although the application of the Berendsen thermostat to remove energy near the transition region did reduce the energy increase, it was not sufficient to allow the method to model accurately any real nanoindentation experiment.

The force attribution method described in section 6.5 was developed to resolve the energy increase in the position matching method. The method strictly obeys Newton's third law of motion such that equal and opposite forces are applied between all particles in the atomistic, transition, and continuum region, guaranteeing conservation of energy. The results from the indentation test simulation for this model showed this to be the case, and that the induced strain field due to the indentation passed smoothly across the transition region into the continuum region. Hence, in chapter 7 , the force attribution coupling method was used to model nanoindentation and laser ablation simulations to investigate the effects of adding the embedding continuum region.

The results from the simulations in chapter 7 show that the addition of the embedding continuum region in the coupled model allows long range strain fields and heat waves to be modelled in the far field by continuum mechanics. 
Kinetic energy, due to nanoindentation or laser ablation, is transferred from the atomistic region to the continuum region to give a more realistic description of the experiment than atomistic only simulations, where the energy is artificially constrained to the atomistic region. This may remove the need for temperature control in nanoindentation simulations, which in the past has been required to control excess heating of the substrate.

The contact pressure value from the coupled model for both the $\mathrm{Au}$ and Fe indentation simulations indicates that neglecting to model elastic compression in the far field may cause artificially high values for contact pressure in atomistic only simulations with fixed boundary conditions.

\subsection{Future work}

Although the simulations using the multiscale model already represent larger systems than would normally be modelled in atomistic only simulations, these initial results are only an example of the capability of the coupled model. The new method has the potential to investigate even larger and more realistic simulations and work is currently underway in this direction where the force attribution coupled model will be combined with an existing parallel MD code and applied to nanocrystalline materials and thin film structures to compare to experimental work.

The method has so far only been applied to fcc and bcc crystal structures. However, there are plans to use the coupled model to link to a wider variety of crystal and amorphous structures. As mentioned previously, the force attribution method has an advantage over other methods, that there does not need to be a direct match between nodes and atoms, making the linking far easier when considering complicated atomic arrangements. 
The coupled model currently describes the far field effects in the continuum region by linear elastic finite elements. Although this has proved a reasonable approximation to the solution for the small indentation depths that have been simulated so far, it may be necessary in the future to implement a more accurate finite element description of the continuum region, such as a nonlinear elasticity model, to simulate deeper indentation depths.

A natural progression from the nanoindentation experiment which has been investigated in this work, is to study multiscale modelling of nanotribology experiments $[56,49]$. The multiscale model will allow the long range effects of scratching to be modelled in the continuum region, and as in the nanoidentation simulations, the modelling of kinetic energy dissipation into the far field more realistically describes the experiment.

Ceramic materials with charged particles, such as $\mathrm{MgO}$ or spinel, have interatomic forces evaluated over the whole body of material. With such materials, it is not straightforward to incorporate the required potential function, which describes the interactions, into the coupled model because of the long ranged nature of the Coulomb field and this is an area for future research. For interatomic potential functions which have long range force fields over the whole body of material, it may be possible to apply some finite cut-off distance at the edge of the atomistic region as part of the approximate nature of the method. A more accurate solution in these cases may be to use a new technique involving a potential function derived via a neural network [57] which does have a finite cut-off distance, and could therefore be applied to the coupled model.

The simulations studied in this thesis have modelled applications where the distortion generated in the atomistic region has propagated into the continuum region. However, the method is not restricted to these types of 
application and could be applied to experiments where external forces cause motion in the continuum region such as yield strength testing of wires and shear problems. In these experiments, the distortion in the continuum region causes a breakdown in the atomic structure in the atomistic region because of weaknesses in the material due to dislocation motion and defects such as grain boundaries.

All of the multiscale modelling techniques discussed in this work have restricted plastic deformation to the atomistic region. In these models the continuum region has been used to model only elastic deformation in the far field, thus removing the fixed boundaries of the problem much further from the region of plastic deformation. However, some plastic phenomena which travel quickly over large distances, such as dislocation emission in nanoindentation experiments due to pressure under the tip, cannot be modelled by the linear elastic finite element method in the continuum region. To describe realistically material behaviour, a technique is required to model such plastic deformation in the far field. The two possible ways to do this are, to continue to model the plastic deformation by atomistics, where redefinition of the atomistic-continuum boundary and mesh adjustment are required to follow the plastic deformation into the far field, or to allow plasticity in the continuum via a technique such as the coupled atomistic and discrete dislocation plasticity (CADD) method [58].

Like the other methods described in this work, the CADD method is a multiscale model, treating particle interactions by atomistic and continuum mechanics depending on which region they are in, however, unlike the previous methods, it models dislocations not only in the atomistic region but also describes dislocation defects in the continuum region which move in response to stress. The CADD method is currently a $2 \mathrm{D}$ model due to the difficulty 
in transferring the dislocations across the coupled interface in $3 \mathrm{D}$, however further development of this model may be necessary in the future to model deeper indentation depths and hence larger regions of plastic deformation. 


\section{Bibliography}

[1] X.Q. Huang and A.A. Pelegri. J. Composite Mater., 40:1393, 2006.

[2] D. Christopher, R. Smith, and A. Richter. Nanotechnology, 12:372, 2001.

[3] R. Smith, D. Christopher, S. D. Kenny, A. Richter, and B. Wolf. Phys. Rev. B, 67:245405, 2003.

[4] D. Christopher, R. Smith, and A. Richter. Nucl. Instr. and Meth. in Phys. Res. B, 180:117, 2001.

[5] C. F. Sanz-Navarro, S. D. Kenny, and Roger Smith. Nanotechnology, 15:692-697, 2004.

[6] W. C. Swope, H. C. Anderson, P.H. Berens, and K.R. Wilson. J. chem. Phys., 79:5029-33, 1982.

[7] M. P. Allen and D. J. Tildesley. Computer Simulations of Liquids. Oxford University Press, 1987.

[8] R. W. Hockney and J.W. Eastwood. Computer simulations using particles. McGraw-Hill, 1981.

[9] J. E. Lennard-Jones. Proc. Roy. Soc. A, 106:441, 1924.

[10] M. S. Daw and M. I. Baskes. Phys. Rev. B, 29:6443, 1984. 
[11] G. J. Ackland, G. Tichy, V. Vitek, and M. W. Finnis. Phil. Mag A, 56:735-756, 1987.

[12] G. J. Ackland, M. I. Mendelev, D.J. Srolovitz, S. Han, and A. V. Barashev. J. Phys. Condensed Matter, 16:S2629, 2004.

[13] J. Tersoff. Phys. Rev. B, 37:6991, 1988.

[14] J. Tersoff. Phys. Rev. B, 39:5566, 1989.

[15] K. Beardmore and R. Smith. Phil. Mag A, 74:1439, 1996.

[16] D. W. Brenner. Phys. Rev. B, 42:9458, 1990.

[17] D. W. Brenner. Phys. Rev. B, 46:1948, 1992.

[18] J. Ziegler, J. P. Biersack, and U. Littmark. The Stopping and Range of Ions in Solids, volume 1. Pergamon, 1985.

[19] I. S. Duff, R. G. Grimes, and J. G. Lewis. ACM Transactions on Mathematical Software, 15:1-14, 1989.

[20] W. C. Oliver and G. Pharr. J. Mater. Res., 7:1562, 1992.

[21] I. N. Sneddon. Int. J. Engng. Sci., 3:47, 1965.

[22] S. Dub, N. V. Novikov, and Y. V. Milman. Sverkhtverdye Materialy, $3: 31,2002$.

[23] H. J. C. Berendsen, J. P. M. Postma, W. F. Van Gunsteren, A. Di Nola, and J. R. Haak. J. chem. Phys., 81:3684, 1984.

[24] S. Nose. J. chem. Phys., 81:511, 1984.

[25] W. G. Hoover. Molecular Dynamics. Springer-Verlag, 1986. 
[26] A. Richter, R. Ries, and R. Smith. Diam. Rela. Mater., 9:170, 2000.

[27] D. Ge, A. M. Minor, E. A. Stach, and J. W. Morris Jr. Phil. Mag., $86: 4069,2006$.

[28] W. A. Curtin and R. E. Miller. Modelling Simul. Mater. Sci. Eng., 11:R33-R68, 2003.

[29] P. C. Gehlen, G. T. Hahn, and M. F. Kanninen. Scr. Meter., 6:1087, 1972.

[30] P. C. Gehlen. Scr. Meter., 7:1115, 1973.

[31] J. E. Sinclair. Phil. Mag., 31:647, 1975.

[32] S. Kohlhoff, P. Gumbsch, and H. F. Fischmeister. Phil. Mag. A, 64:851$78,1991$.

[33] J. Q. Broughton, F. F. Abraham, Noam Bernstein, and Efthimios Kaxiras. Phys. Rev. B, 60:2391-403, 1999.

[34] R. E. Rudd and J. Q. Broughton. Physica Status Solidi B, 217:251-291, 2000 .

[35] E. B. Tadmor, M. Ortiz, and R. Phillips. Phil. Mag. A, 73:1529-63, 1996.

[36] R. E. Miller and E. B. Tadmor. J. Comput.-Aided Mater. Des., 2003.

[37] F. H. Stillinger and T. A. Weber. Phys. Rev. B, 31:5262, 1985.

[38] E. B. Tadmor, R. Miller, R. Phillips, and M. Ortiz. J. Mater. Res., 14:2233, 1999. 
[39] G. S. Smith, E. B. Tadmor, and E. Kaxiras. Phys. Rev. Lett., 84:1260, 2000 .

[40] G. S. Smith, E. B. Tadmor, N. Bernstein, and E. Kaxiras. Acta Mater., 49:4089, 2001.

[41] J. L. Ericksen. Phase Transformation and Material Instabilities in Solids. New York:Academic, 1984.

[42] J. H. Weiner. Statistical Mechanics of Elasticity. New York:Wiley, 1983.

[43] F. Milstein. J. Mater. Sci, 15:1071, 1980.

[44] V. B. Shenoy, R. Miller, E. B. Tadmor, D. Rodney, R. Phillips, and M. Ortiz. J. Mech. Phys. Solids, 47:611-42, 1998.

[45] K. E. Sickafus, J. M. Wills, and N. W. Grimes. J. Am. Ceram. Soc., 82:3279, 1999.

[46] Y. K. Cheung and M. F. Yeo. A Practical Introduction to Finite Element Analysis. Pitman, 1979.

[47] C. R. F. Maunder. Algebraic Topology. VNR London, 1970.

[48] Tirupathi R. Chandrupatla and Ashok D. Belegundu. Introduction To Finite Elements in Engineering. Prentice-Hall, second edition, 1997.

[49] D. Mulliah, S. D. Kenny, E. McGee, R. Smith, A. Richter, and B. Wolf. Nanotechnology, 17:1807-1818, 2006.

[50] K. Durst, B. Baekes, O. Franke, and M. Goken. Acta Mat., 54:2547$2555,2006$. 
[51] W. W. Gerberich, N. I. Tymiak, J. C. Grunlan, M. F. Horstemeyer, and M. I. Baskes. J. Appl. Mech. - Trans. ASME, 69:433-442, 2002.

[52] R. Bohme, S. Pissadakis, D. Ruthe, and K. Zimmer. App. Phys. AMater. Sci. and Proc., 85:75, 2006.

[53] J. Kaspar, A. Luft, S. Nolte, M. Will, and E. Beyer. J. of Laser App., $18: 85,2006$.

[54] M.N.R. Ashfold, F. Claeyssens, G.M. Fuge, and S.J. Henley. Chem. Soc. Rev., 23:23, 2004.

[55] F. Claeyssens, C.L. Freeman, N.L. Allan, Ye Sun, M.N.R. Ashfold, and J.H. Harding. J. Mater. Chem., 15:139, 2004.

[56] R. Smith, D. Mulliah, S. D. Kenny, E. McGee, A. Richter, and M. Gruner. Wear, 259:459, 2005.

[57] A. Bholoa. Potential Energy Surfaces Using Neural Networks. PhD thesis, Loughborough University, 2006.

[58] L. E. Shilkrot, R. E. Miller, and W. A. Curtin. Phys. Rev. Lett., 89:025501-1-025501-4, 2002. 
